\title{
Suzuki reaction on pyridinium $N$-haloheteroarylaminides: regioselective synthesis of 3,5-disubstituted 2-aminopyrazines
}

\author{
Rafael Castillo, M. José Reyes, M. Luisa Izquierdo, Julio Alvarez-Builla* \\ Departamento de Química Orgánica, Universidad de Alcalá, 28871 Alcalá de Henares, Madrid, Spain
}

Received 1 October 2007; received in revised form 15 November 2007; accepted 16 November 2007

Available online 22 November 2007

Dedicated to Professor M. Yus on the occasion of his 60th birthday

\begin{abstract}
An extensive study of Suzuki-Miyaura cross-coupling processes on $N$-pyridinium bromoazinyl aminides has been performed. Mono- and disubstitution on 5- and 3,5-bromo derivatives produced the corresponding aryl derivatives. In the disubstituted compounds regioselective substitution at the 3-position occurred, vicinal to the aminide nitrogen, and this was more evident in pyrazine derivatives. The commonly used strategy involving $\mathrm{N}$-alkylation and reduction of the $\mathrm{N}-\mathrm{N}$ bond gave rise to a series of 2-alkylamino-3,5-disubstituted-pyrazines.
\end{abstract}

(C) 2007 Elsevier Ltd. All rights reserved.

\section{Introduction}

Polysubstituted heterocycles belong to the most important class of organic compounds, they are widespread in nature and many of them show interesting biological activities. ${ }^{1}$ Functionalization of halo- or metallated heteroaromatic compounds through metal-mediated cross-coupling reactions has become an important method for the preparation of different multiple carbon-substituted heterocycles ${ }^{2}$ and, in recent years, selective Pd-mediated reactions have been developed to prepare highly functionalized heterocycles. ${ }^{3}$ The SuzukiMiyaura cross-coupling reaction has proved to be a powerful and versatile method for the formation of $\mathrm{Csp}^{2}-\mathrm{Csp}^{2}$ bonds. ${ }^{4}$ The process has important advantages over the other palladium-catalyzed cross-coupling processes and these include functional group compatibility, low toxicity of reagents and intermediates, ready availability of boron derivatives, high thermal stability and good tolerance against oxygen and aqueous solvents. Recently, efforts have been focused on the development of efficient and selective catalytic systems that either

\footnotetext{
* Corresponding author. Tel.: +34 9188546 06; fax: +34 918854686 . E-mail address: julio.alvarez@uah.es (J. Alvarez-Builla).
}

allow the use of chlorinated substrates or avoid the use of organic solvents. ${ }^{5}$

Pyridinium heteroaryl-stabilized aminides 1 (Fig. 1), in which we have been interested for a number of years, ${ }^{6}$ are a subgroup of mesomeric betaines ${ }^{7}$ with interesting reactivity ${ }^{8}$ due to their particular structure. ${ }^{8 b}$

Regioselective halogenation ${ }^{8 c}$ at the iminoazine moiety affords 3- or 3,5-halogenated aminides and these compounds have been used in radical cyclization processes ${ }^{9}$ and, more recently, preliminary results have been reported for the Suzuki cross-coupling reaction of compounds $\mathbf{2 a}, \mathbf{2} \mathbf{b}$ and $\mathbf{2 d}$ (Fig. 1) with boronic acids. ${ }^{10}$

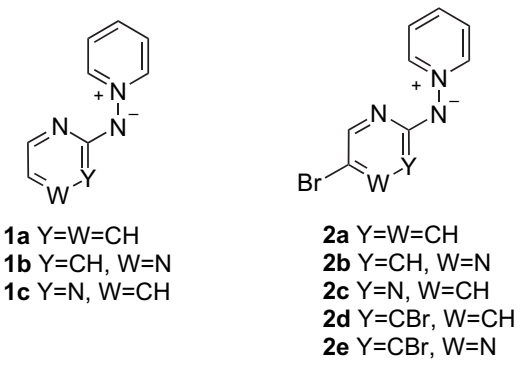

Figure 1. 


\section{Results and discussion}

\subsection{Monosubstituted N-aminides}

The reaction of pyridinium $N$-(5-bromoheteroaryl)aminides 2a, $\mathbf{2 b}$ and boronic acids worked effectively under standard conditions: i.e., aminide ( $1 \mathrm{mmol})$, boronic acid $(1.5 \mathrm{mmol})$, $\mathrm{Pd}\left(\mathrm{PPh}_{3}\right)_{4}(5 \mathrm{mmol} \%)$ and $\mathrm{Cs}_{2} \mathrm{CO}_{3}(2 \mathrm{mmol})$ in refluxing toluene/ethanol $(20: 1, \mathrm{v} / \mathrm{v})^{10 \mathrm{a}}$ (Table 1 , method A) to afford the substituted products $\mathbf{3}$ and $\mathbf{4}$. In all cases the process was continued until substrate $\mathbf{2}$ had been consumed. In addition, in the first attempt to prepare compound 3d it was found that 4-(hydroxymethyl)phenylboronic acid had low solubility in toluene and, as a result, the ratio of toluene/ethanol had to be optimized, yielding $73 \%$ of $\mathbf{3 d}$ with a $4: 1$ mixture of toluene/ethanol. Moreover, it is known that the base plays an important role in the Suzuki process, ${ }^{4 \mathrm{a}}$ with the amount and base strength being two important factors in the transmetallation step in terms of increasing the conversion yields. As in previous work, the use of hard bases ${ }^{10 a}$ did not increase the yield of 3a but a softer base, such as $\mathrm{K}_{2} \mathrm{CO}_{3}$, was tested in large excess ${ }^{11}$ in the coupling of aminide $\mathbf{2 a}$ with different boronic acids. In most cases, the method (Table 1, method B, with $10 \mathrm{mmol}$ of base) produced high yields, often with shorter reaction times, particularly in the case of $\mathbf{3 d}$, which was obtained in excellent yield $(92 \%)$ (Scheme 1).

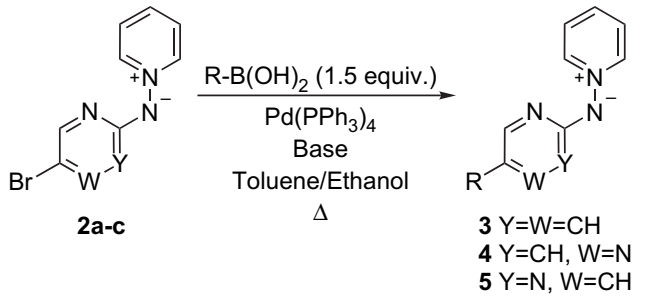

Scheme 1.

Results for derivatives of pyridine, pyrazine and pyrimidine (compounds $\mathbf{3} \mathbf{- 5}$, respectively) are given in Table 1 .

\subsection{Disubstituted N-aminides}

Dibromoaminide $\mathbf{2} \mathbf{d}^{8 \mathrm{c}}$ was tested in a double Suzuki process, using 3 equiv of boronic acid, 4 equiv of $\mathrm{Cs}_{2} \mathrm{CO}_{3}$ and a mixture of toluene/ethanol (20:1, v/v) under reflux ${ }^{10 a}$ (Table 2 , method A); alternatively, bearing in mind the previous results (Table 1, method B) a double coupling was also tested on pyridinium $\mathrm{N}$-(3,5-dibromoazin-2-yl)aminides $\mathbf{2 d}$ and $\mathbf{2 e}$ and several boronic acids, using 20 equiv of $\mathrm{K}_{2} \mathrm{CO}_{3}$ in a toluene/ethanol (4:1) solvent mixture under reflux (Table 2). Yields were again higher on using method B and compounds 6 and 7 were obtained, as shown in Table 2.

Unexpectedly, $\mathbf{6 h}$ was obtained in low yield (18\%) together with the monosubstituted compound $\mathbf{8}$ as the main product $(48 \%)$ and $28 \%$ of the starting material $2 d$. This result indicates a possible preference in the coupling process that gives rise to compound $\mathbf{8}$ rather than its isomer $\mathbf{9}$-the expected compound if steric effects were predominant in the process (Scheme 2). ${ }^{10 b}$

Monocoupling of $\mathrm{N}$-aminides appeared to be even more efficient in pyrazine derivatives $\mathbf{2} \mathbf{a}-\mathbf{2 c}$. As a consequence, ${ }^{10 b}$ a method involving a modification of method $\mathrm{B}$ was developed in order to decrease the amount of boronic acids $(1.1 \mathrm{mmol})$ required with the aim of minimizing double coupling (Table 3). A series of 3-aryl or heteroaryl $N$-(5-bromopyrazin-2-yl)pyridinium aminides $\mathbf{1 0}$ were obtained. Although compounds $\mathbf{1 0}$ were obtained in fairly good yield, traces of diaryl aminides 7 and $5^{\prime}$-aryl products were detected in the reaction mixture. A reduction in the amount of catalyst from 5 to $1 \mathrm{~mol} \%$ minimized the insertion of $\mathrm{Pd}^{0}$ into the $\mathrm{C}-\mathrm{Br}$ bond in the $5^{\prime}$-position and, although the reaction took place slowly, a considerable increase in selectivity and therefore in yield was observed (Table 3, compounds 10a and 10c).

A model for the intermediate, in which the unshared pair of the aminide nitrogen coordinates palladium to provide some extra stability to the complex, has been proposed to explain the observed selectivity (Scheme 3 ). ${ }^{10 b}$

A similar type of behaviour has been reported in the literature for Pd-mediated processes involving 3,5-dibromopyrazines that contain substituents with an unshared electron pair in the 2-position. ${ }^{3 b, 12}$ In addition, the recent regioselective reduction of 3,5-dibromopyrazin-2-ylamine produced only 5-bromo-pyrazin-2-ylamine on using various hydride donors, palladium sources, ligands, bases and solvents. ${ }^{12 \mathrm{f}}$ The authors suggest the participation of the neighbouring amino group as a ligand as an explanation for the observed regioselectivity.

Once monocoupled aminides $\mathbf{1 0}$ had been prepared, a second coupling on the remaining bromine in the 5-position was achieved under previously described conditions (method B) to give almost quantitative yields of the unsymmetrical $3^{\prime}, 5^{\prime}$-disubstituted pyrazin-2-ylaminides 11 (Scheme 4) reported in Table 4.

Two significant differences in chemical shifts can be observed in the ${ }^{1} \mathrm{H}$ NMR spectra of $N$-( $3^{\prime}, 5^{\prime}$-diarylpyrazin-2-yl)pyridinium aminides $\mathbf{7}$ and $\mathbf{1 0}$ in relation to the position of the aryl (or heteroaryl) substituent on the diazine ring: (a) substituents in the 5-position exert a deshielding effect on H6 $(\sim 0.5 \mathrm{ppm})$ and (b) the signals for the $\alpha$-protons of the group in the $3^{\prime}$-position appear at lower field than those for the same substituent on the $5^{\prime}$-position. Representative chemical shifts for compounds $\mathbf{2 d}, \mathbf{4 a}, 7 \mathbf{a}$ and 10a are given as examples in Figure 2.

\section{Synthesis of 2-alkylaminoazines}

The chemistry of pyridinium $N$-aminides has been well developed in our research group, and several publications refer to a regioselective $\mathrm{N}$-alkylation and the subsequent reduction of the $\mathrm{N}-\mathrm{N}$ bond in order to obtain 2-alkylaminoazines. ${ }^{8 \mathrm{~b}, \mathrm{e}, \mathrm{g}, \mathrm{i}}$ The preparation of 2-aminoazines is interesting not only because they are valuable intermediates in synthesis but also due to their interesting properties. ${ }^{12}$ However, the preparation of highly functionalized 2-aminopyrazines is not an easy task. ${ }^{13,14}$ The present study could form the basis of a good 
Table 1

Arylation of aminides $\mathbf{2 a}-\mathbf{c}$ with different arylboronic acids ${ }^{\mathrm{a}}$

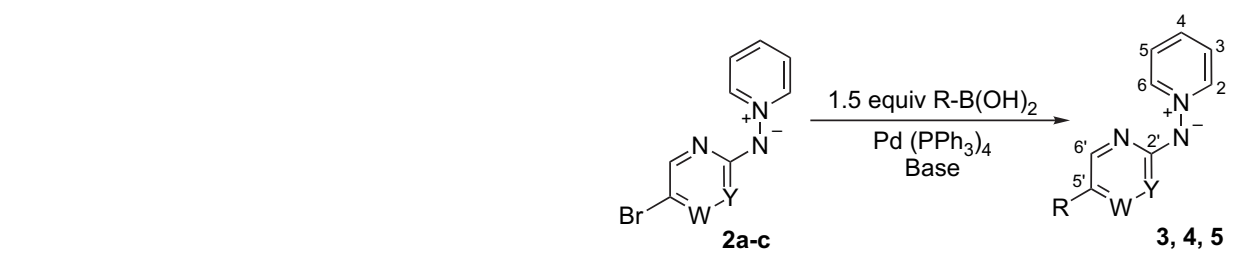

\begin{tabular}{|c|c|c|c|c|c|c|c|c|c|c|c|c|c|}
\hline $\mathrm{R}^{\mathrm{a}}$ & Compound & W & $\mathrm{Y}$ & Method $^{\mathrm{b}}$ & $\begin{array}{l}\text { Time } \\
\text { (h) }\end{array}$ & $\begin{array}{l}\text { Yield } \\
(\%)\end{array}$ & $\mathrm{R}^{\mathrm{a}}$ & Compound & W & $\mathrm{Y}$ & Method $^{\mathrm{b}}$ & $\begin{array}{l}\text { Time } \\
\text { (h) }\end{array}$ & $\begin{array}{l}\text { Yiel } \\
(\%)\end{array}$ \\
\hline & $3 \mathbf{a}$ & $\mathrm{CH}$ & $\mathrm{CH}$ & $\begin{array}{l}\text { A } \\
\text { B }\end{array}$ & $\begin{array}{l}8 \\
8\end{array}$ & $\begin{array}{l}90 \\
95\end{array}$ & & 31 & $\mathrm{CH}$ & $\mathrm{CH}$ & A & 2 & 95 \\
\hline
\end{tabular}

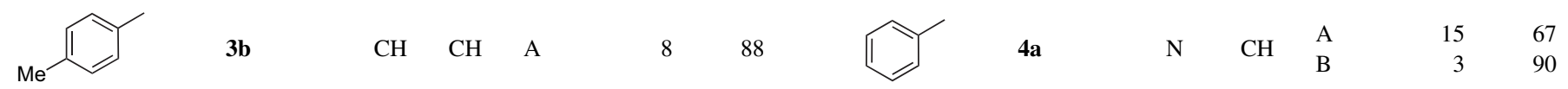

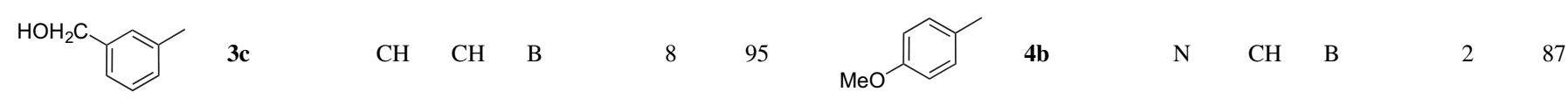

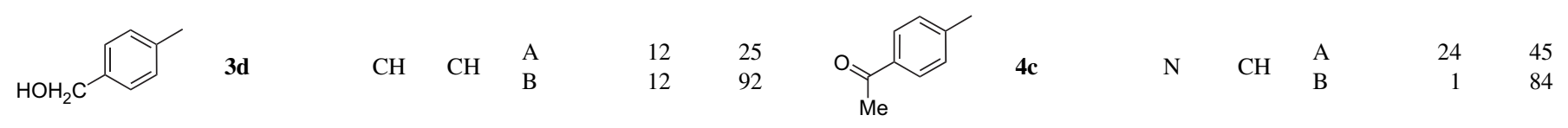

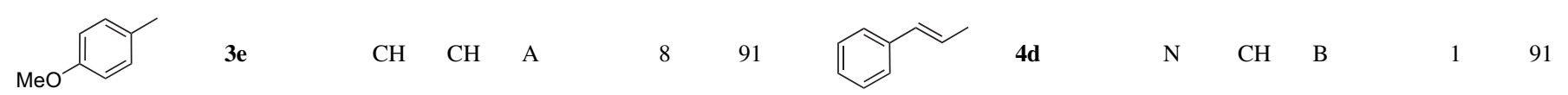

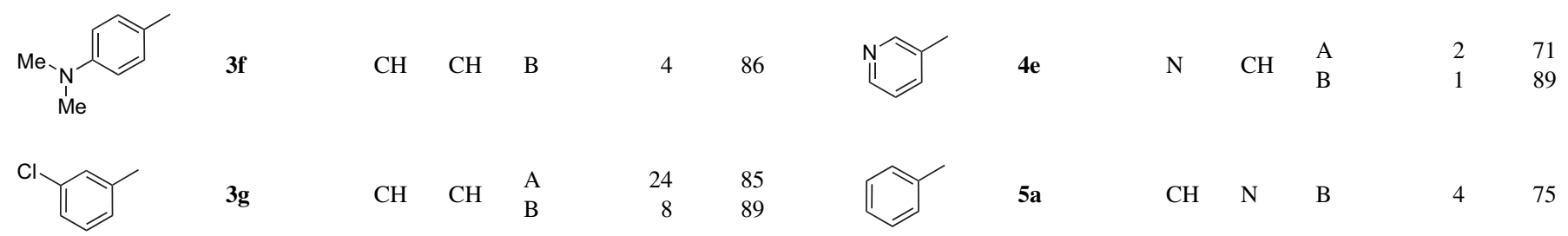

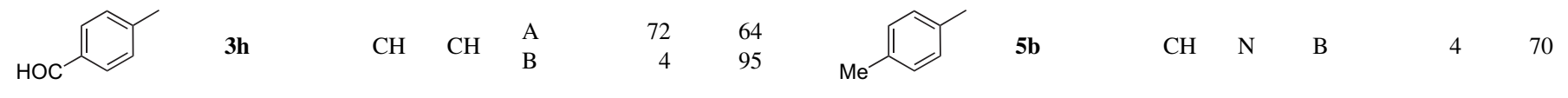<smiles>COc1ccc(C)cc1</smiles><smiles>CC=Cc1ccc(F)cc1</smiles>

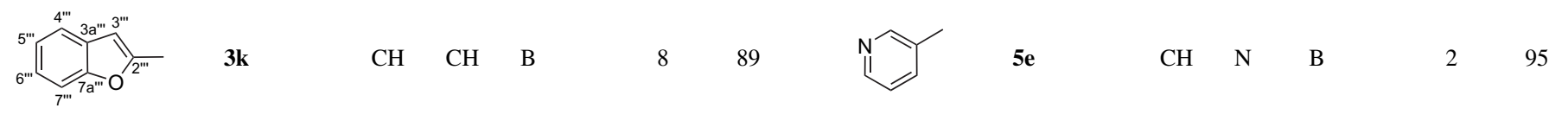

a Ring numbering employed in NMR analysis.

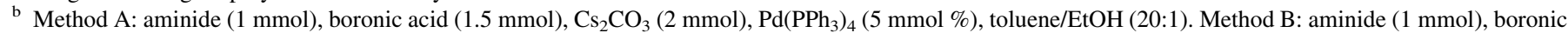
acid $(1.5 \mathrm{mmol}), \mathrm{K}_{2} \mathrm{CO}_{3}(10 \mathrm{mmol}), \mathrm{Pd}\left(\mathrm{PPh}_{3}\right)_{4}(5 \mathrm{mmol} \%)$, toluene/EtOH (4:1). 
Table 2

Diarylation of aminides $\mathbf{2 d}$,e with different boronic acids

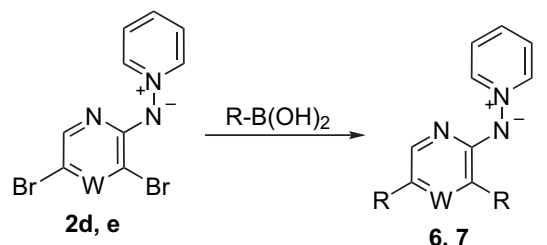

\begin{tabular}{|c|c|c|c|c|c|c|c|c|c|c|c|}
\hline $\mathrm{R}$ & Compound & W & Method $^{\mathrm{a}}$ & Time (h) & Yield (\%) & $\mathrm{R}$ & Compound & W & Method $^{\mathrm{a}}$ & Time (h) & Yield $(\%)$ \\
\hline & $6 a$ & $\mathrm{CH}$ & A & 8 & 63 & & $7 a$ & $\mathrm{~N}$ & $\begin{array}{l}\text { A } \\
\text { B }\end{array}$ & $\begin{array}{r}15 \\
8\end{array}$ & $\begin{array}{l}67 \\
90\end{array}$ \\
\hline & $6 b$ & $\mathrm{CH}$ & A & 8 & 56 & & $7 b$ & $\mathrm{~N}$ & B & 2 & 86 \\
\hline & $6 c$ & $\mathrm{CH}$ & B & 8 & 86 & & $7 c$ & $\mathrm{~N}$ & $\begin{array}{l}\text { A } \\
\text { B }\end{array}$ & $\begin{array}{r}24 \\
8\end{array}$ & $\begin{array}{l}45 \\
95\end{array}$ \\
\hline & $6 d$ & $\mathrm{CH}$ & A & 3 & 90 & & $7 d$ & $\mathrm{~N}$ & B & 1 & 88 \\
\hline & $6 e$ & $\mathrm{CH}$ & B & 8 & 70 & & $7 e$ & $\mathrm{~N}$ & B & 8 & 98 \\
\hline & $6 f$ & $\mathrm{CH}$ & A & 8 & 78 & & $7 f$ & $\mathrm{~N}$ & B & 8 & 89 \\
\hline & $6 \mathrm{~g}$ & $\mathrm{CH}$ & A & 3 & 85 & & $7 \mathrm{~g}$ & $\mathrm{~N}$ & $\begin{array}{l}\text { A } \\
\text { B }\end{array}$ & $\begin{array}{l}2 \\
8\end{array}$ & $\begin{array}{l}71 \\
89\end{array}$ \\
\hline & $6 \mathrm{~h}$ & $\mathrm{CH}$ & A & 8 & $18^{\mathrm{b}}$ & & & & & & \\
\hline
\end{tabular}

${ }^{\mathrm{a}}$ Method A: boronic acid $(3 \mathrm{mmol}), \mathrm{Cs}_{2} \mathrm{CO}_{3}(4 \mathrm{mmol}), \mathrm{Pd}\left(\mathrm{PPh}_{3}\right)_{4}(5 \mathrm{~mol} \%)$, toluene/ethanol (20:1). Method B: boronic acid $(3 \mathrm{mmol}), \mathrm{K}_{2} \mathrm{CO}{ }_{3}(20 \mathrm{mmol})$, $\mathrm{Pd}\left(\mathrm{PPh}_{3}\right)_{4}(5 \mathrm{~mol} \%)$, toluene/ethanol $(4: 1)$.

b With $48 \%$ of aminide 8 .

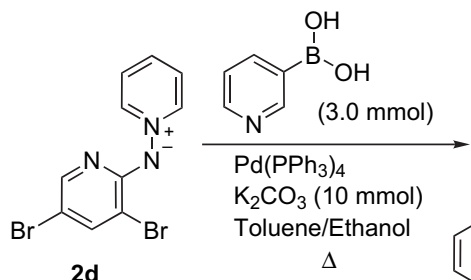

2d

$\Delta$

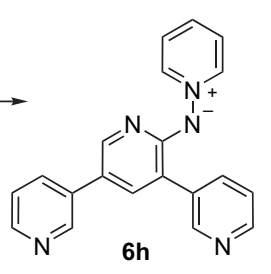

Scheme 2 .<smiles>Brc1cnc(-c2cccnc2)c(-n2cccc2)c1</smiles><smiles>Brc1cc(-c2cccnc2)cnc1-[n+]1ccccc1</smiles>

approach to produce 2-benzylamino-3,5-diarylpyrazines $\mathbf{1 3}$ with a high degree of variability, by reduction of salts $\mathbf{1 2}$ (Scheme 4).

Alkylation of aminides $\mathbf{1 1}$ was performed in anhydrous acetone and, once the solvent had been removed in vacuo, the pyridinium salts $\mathbf{1 2}$ were isolated by treatment with ethyl acetate, in which the excess alkylating agent and the unreacted aminide $\mathbf{1 1}$ remained in solution. The pyridinium salts $\mathbf{1 2}$ were used in the next step without further purification. The yields indicated in Table 5 are the result of the two steps: $\mathrm{N}$-alkylation and $\mathrm{N}-\mathrm{N}$ reduction.
In previous papers, we reported that the nuclear nitrogen on $\mathrm{N}$-pyridin-2-yl pyridinium aminides is partially blocked by an intramolecular hydrogen bond, making the alkylation regioselective on the exo-nitrogen ${ }^{8 b}$ (Fig. 3). Nevertheless, when this process was applied to unsubstituted diazine-stabilized aminides, where only one nitrogen can be blocked by the hydrogen bond, alkylation on the alternative nitrogen of the diazine ring was also detected. ${ }^{8 \mathrm{e}}$ In the case described here, the presence of two bulky aryl substituents at the $3^{\prime}$ - and $5^{\prime}$-position makes $\mathrm{N}^{\prime}$ less accessible, and only the exocyclic nitrogen alkylation product is successfully obtained (Fig. 3). 
Table 3

Monoarylation of $3^{\prime}, 5^{\prime}$-dibromopyrazin-2' -yl pyridinium $N$-aminide $2 \mathbf{e}$

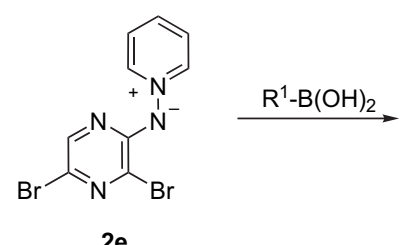<smiles>[R]c1nc(Br)cnc1[N-][n+]1ccccc1</smiles>

10

\begin{tabular}{|c|c|c|c|}
\hline $\mathrm{R}^{1}$ & Compound & Reaction time (h) & Yield (\%) \\
\hline & $10 a$ & $3(48)^{\mathrm{a}}$ & $75(87)^{\mathrm{a}}$ \\
\hline & $10 \mathrm{~b}$ & 5 & 71 \\
\hline & 10c & $8(48)^{\mathrm{a}}$ & $72(85)^{\mathrm{a}}$ \\
\hline & 10d & 8 & 65 \\
\hline & $10 \mathrm{e}$ & 4 & 50 \\
\hline & $10 f$ & 12 & 84 \\
\hline & $10 \mathrm{~g}$ & 12 & 81 \\
\hline & $10 \mathrm{~h}$ & 2 & 65 \\
\hline & $10 \mathrm{i}$ & 4 & 77 \\
\hline & $10 \mathrm{j}$ & 16 & 60 \\
\hline
\end{tabular}

${ }^{\mathrm{a}} \mathrm{Pd}\left(\mathrm{PPh}_{3}\right)_{4}(1 \mathrm{~mol} \%)$.

\section{Conclusions}

In conclusion, the peculiar reactivity of aminides $\mathbf{1}$, which can be selectively mono- or dihalogenated ${ }^{8 \mathrm{c}}$ allows an easy mono- or diarylation through the Suzuki reaction to produce compounds 3-7 with a diverse range of 3- and 3,5-aryl and heteroaryl substituents, all obtained with excellent yields. In most cases, the method employing an excess of $\mathrm{K}_{2} \mathrm{CO}_{3}$ (method B) gives rise to better results than the use of $\mathrm{Cs}_{2} \mathrm{CO}_{3} .{ }^{10 a}$

Moreover, the Suzuki process on dihalo derivatives of pyridine, such as $\mathbf{2 e}$, showed a regioselective arylation-predominantly at the 3 -position of the pyridine. The regioselectivity was higher with pyrazine derivatives and this yielded 3 -aryl derivatives, which were subsequently substituted with a different aryl group, thus yielding unsymmetrical pyridinium $\mathrm{N}$-(3,5-diarylpyrazin-2-yl)aminides $\mathbf{1 1}$ in high yields. Finally, 2-alkylaminopyrazines 13 were prepared by alkylation of aminides 11 followed by $\mathrm{N}-\mathrm{N}$ bond reduction of the corresponding aminopyridinium salts $\mathbf{1 2}$.

In summary, the combination of the Suzuki arylation of pyridinium $N$-(3'-bromo or $3^{\prime}, 5^{\prime}$-dibromoazinyl)aminides and conventional $\mathrm{N}$-alkylation and $\mathrm{N}-\mathrm{N}$ reduction allows the preparation of differently functionalized 2-alkylaminopyrazines, and eventually pyridines or pyrimidines, with high efficiency. Other Pd processes are currently being developed for these broadly useful substrates.

\section{Experimental}

\subsection{General remarks}

All melting points were determined in open capillary tubes on Gallenkamp MFB-595-010 M, Electrothermal LA6304 or Stuart Scientific SMP3 melting point apparatus and are uncorrected. IR spectra were obtained on a Perkin-Elmer FTIR 1725X spectrophotometer. ${ }^{1} \mathrm{H}$ and ${ }^{13} \mathrm{C}$ NMR spectra were recorded on Varian Unity 300 or $500 \mathrm{MHz}$ spectrometer or on

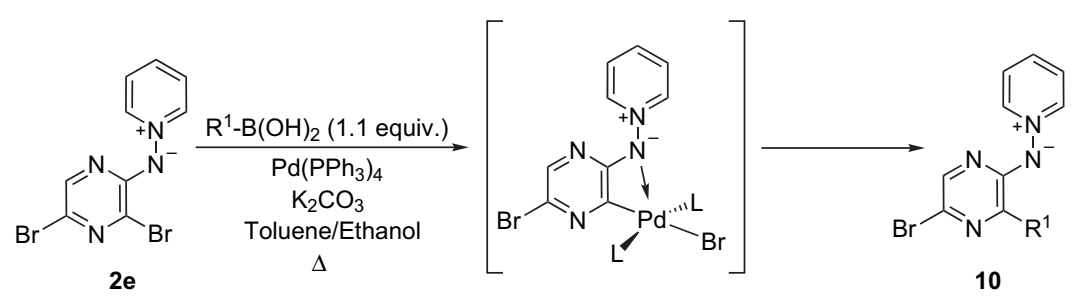

Scheme 3.

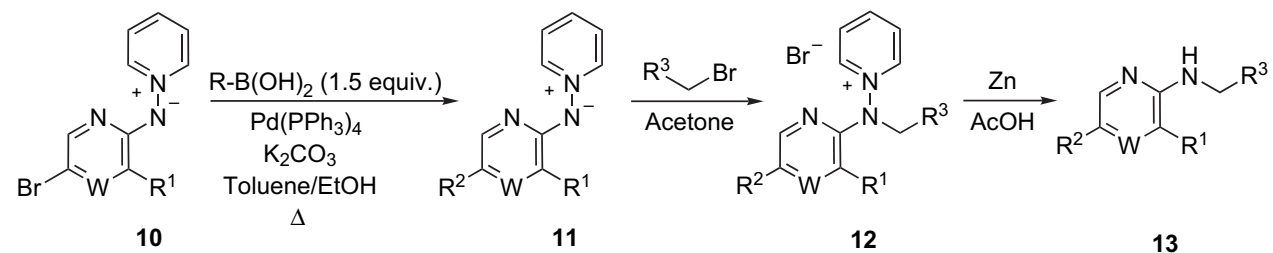

Scheme 4. 
Table 4

Monoarylation of monocoupled aminides $\mathbf{1 0}$

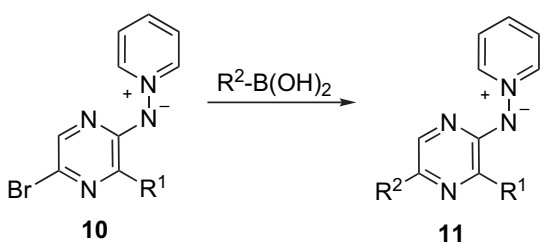

\begin{tabular}{|c|c|c|c|c|}
\hline $\mathrm{R}^{1 \mathrm{a}}$ & $\mathrm{R}^{2 \mathrm{~b}}$ & Compound & Reaction time (h) & Yield $(\%)$ \\
\hline & & $11 a$ & 8 & 99 \\
\hline & & $11 b$ & 8 & 95 \\
\hline & & 11c & 8 & 89 \\
\hline & & 11d & 8 & 84 \\
\hline & & $11 \mathrm{e}$ & 1 & $92^{\mathrm{c}}$ \\
\hline & & $11 f$ & 1 & $91^{\mathrm{c}}$ \\
\hline & & $11 \mathrm{~g}$ & 8 & 90 \\
\hline & & $11 \mathrm{~h}$ & 8 & 82 \\
\hline & & $11 \mathbf{i}$ & 8 & 81 \\
\hline & & $\mathbf{1 1 j}$ & 8 & 65 \\
\hline & & $11 k$ & 8 & 90 \\
\hline & & 111 & 8 & 86 \\
\hline & & $11 \mathrm{~m}$ & 8 & 90 \\
\hline
\end{tabular}

\footnotetext{
a Positions labelled as (") for NMR analysis.

b Positions labelled as ("') for NMR analysis.

c Yield has been improved in comparison to the first published results. ${ }^{10 a}$
}

a Varian Mercury VX-300 system at room temperature. Chemical shifts are given in parts per million $(\delta)$ downfield from TMS. Coupling constants $(J)$ are in hertz $(\mathrm{Hz})$ and signals are described as follows: s, singlet; $\mathrm{d}$, doublet; $\mathrm{t}$, triplet; $\mathrm{m}$, multiplet; br, broad; ap, apparent. The tentative assignment of proton and carbon resonances has been made on the basis of double resonance, NOESY and TOCSY experiments, two- dimensional $\mathrm{H}, \mathrm{H}$ and $\mathrm{H}, \mathrm{C}$-correlation experiments, COSY, HSQC, HMBC or related spectra. In order to identify the signals in the description of each product, positions of the pyridinium ring are not labelled, positions of the azine ring are labelled $\left(^{\prime}\right)$, positions of the 3-aryl substituent are labelled $\left(^{\prime \prime}\right)$, positions of the 5-aryl substituent ('I') and positions of the aryl belonging to the aryl ring of the $N$-benzyl substituent, when present, are labelled $\left(^{\prime \prime \prime \prime}\right)$. Elemental analyses were carried out on a Heraeus Rapid CHN analyzer and were within $0.4 \%$ of the theoretical values for all the new compounds described. Low resolution mass spectra (MS) were taken on a HewlettPackard 5988A (70 eV) spectrometer using chemical ionization (CI) or electrospray (ESI) and high resolution analysis (FAB or TOF) was performed on a VG AutoSPEC (Micromass Instrument) or on an Agilent 6210 Time-of-flight LC/MS. All reagents and solvents were obtained from commercial sources, except 3-pyridineboronic acid, ${ }^{15}$ and were used without further purification. TLC analyses were performed on silica gel (Kieselgel $60 \mathrm{~F}_{254}$, Macherey-Nagel) and spots were visualized under UV light. Column chromatography was carried out with silica gel $60(40-63 \mu \mathrm{m}$, Merck) and/or employing Biotage columns, using the mobile phase reported for each case.

\subsection{General procedures for the preparation of 5-substituted $N$-(azin-2-yl)pyridinium aminides (3-5)}

Method A. Aminides $\mathbf{2 a}-\mathbf{2} \mathbf{c}^{8 \mathrm{c}}$ (1 $\left.\mathrm{mmol}\right)$, the corresponding boronic acid $(1.5 \mathrm{mmol})$ and $\mathrm{Cs}_{2} \mathrm{CO}_{3}(2 \mathrm{mmol})$ were dissolved in a toluene/ethanol mixture $(20: 1,15 \mathrm{~mL}) \cdot \mathrm{Pd}\left(\mathrm{PPh}_{3}\right)_{4}(5 \mathrm{mmol} \%)$ was added and the mixture was stirred under argon and heated under reflux for the reaction time indicated in Table 1.

Method B. Aminides $\mathbf{2 a}-\mathbf{2} \mathbf{c}^{8 \mathrm{c}}$ (1 $\left.\mathrm{mmol}\right)$, the corresponding boronic acid $(1.5 \mathrm{mmol})$ and $\mathrm{K}_{2} \mathrm{CO}_{3}(10 \mathrm{mmol})$ were dissolved in a toluene/ethanol mixture $(20: 1,15 \mathrm{~mL}) . \mathrm{Pd}\left(\mathrm{PPh}_{3}\right)_{4}(5 \mathrm{mmol} \%)$ was added and the mixture was stirred under argon and heated under reflux for the reaction time indicated in Table 1.

In both cases, the course of the reaction was followed by TLC, HPLC and/or ${ }^{1} \mathrm{H}$ NMR spectroscopy. Once the starting material had been consumed, the system was allowed to reach room temperature, the mixture was filtered through Celite or silica gel and washed with acetonitrile until colour was no longer observed in the filtrate. The combined filtrates were evaporated to dryness. The crude product was purified by flash chromatography on a silica gel column, with ethanol as the mobile phase, and recrystallized from a suitable solvent. Compound $\mathbf{3 l}$ was isolated as a yellow oil, while compounds $\mathbf{3 a}, \mathbf{3 c}$, $\mathbf{3 g}, \mathbf{4 c}, \mathbf{4 e}$ and $\mathbf{5 a}-\mathbf{5 e}$ were transformed into the corresponding hydrobromides before purification.

\subsubsection{N-(5-Phenylpyridin-2-yl)pyridinium aminide (3a)}

See Ref. 10a.

\subsubsection{N-[5-(4-Methylphenyl)pyridin-2-yl]pyridinium} aminide $(\mathbf{3 b})$

Orange solid (300 mg, 88\%, ethyl acetate/hexane), mp 141$142{ }^{\circ} \mathrm{C}$; IR $(\mathrm{KBr}) \nu_{\max }\left(\mathrm{cm}^{-1}\right): 1594,1468,1376,1287,1130$, 809, 670; ${ }^{1} \mathrm{H}$ NMR (300 MHz, $\left.\mathrm{CD}_{3} \mathrm{OD}\right): \delta 8.75(2 \mathrm{H}, \mathrm{dd}$, 


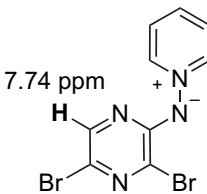

2d

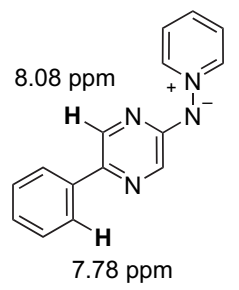

$4 a$
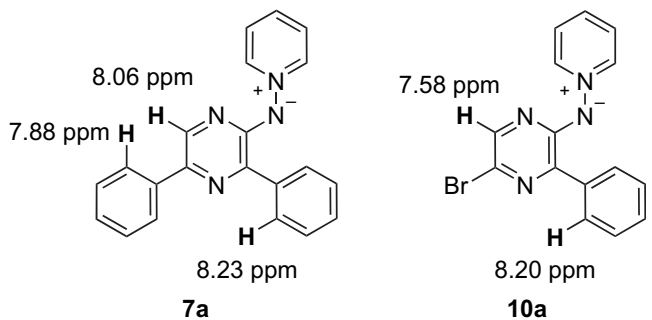

Figure 2.
$J=7.0$ and $1.3 \mathrm{~Hz}, H 2(6)), 7.96(1 \mathrm{H}, \mathrm{dd}, J=2.5$ and $0.7 \mathrm{~Hz}$, $\left.H 6^{\prime}\right), 7.91(1 \mathrm{H}, \mathrm{tt}, J=7.7$ and $1.3 \mathrm{~Hz}, H 4), 7.71(2 \mathrm{H}, \mathrm{dd}$, $J=7.7$ and $7.0 \mathrm{~Hz}, H 3(5)), 7.65(1 \mathrm{H}, \mathrm{dd}, J=8.9$ and $2.5 \mathrm{~Hz}$, $\left.H 4^{\prime}\right), 7.37\left(2 \mathrm{H}, \mathrm{d}, J=8.0 \mathrm{~Hz}, H 2^{\prime \prime \prime}\left(6^{\prime \prime \prime}\right)\right), 7.17(2 \mathrm{H}, \mathrm{d}$, $\left.J=8.0 \mathrm{~Hz}, H 3^{\prime \prime \prime}\left(5^{\prime \prime \prime}\right)\right), 6.61\left(1 \mathrm{H}, \mathrm{dd}, J=8.9\right.$ and $\left.0.7 \mathrm{~Hz}, H 3^{\prime}\right)$,

Table 5

Synthesis of 3,5-disubstituted aminopyrazines $\mathbf{1 3}$
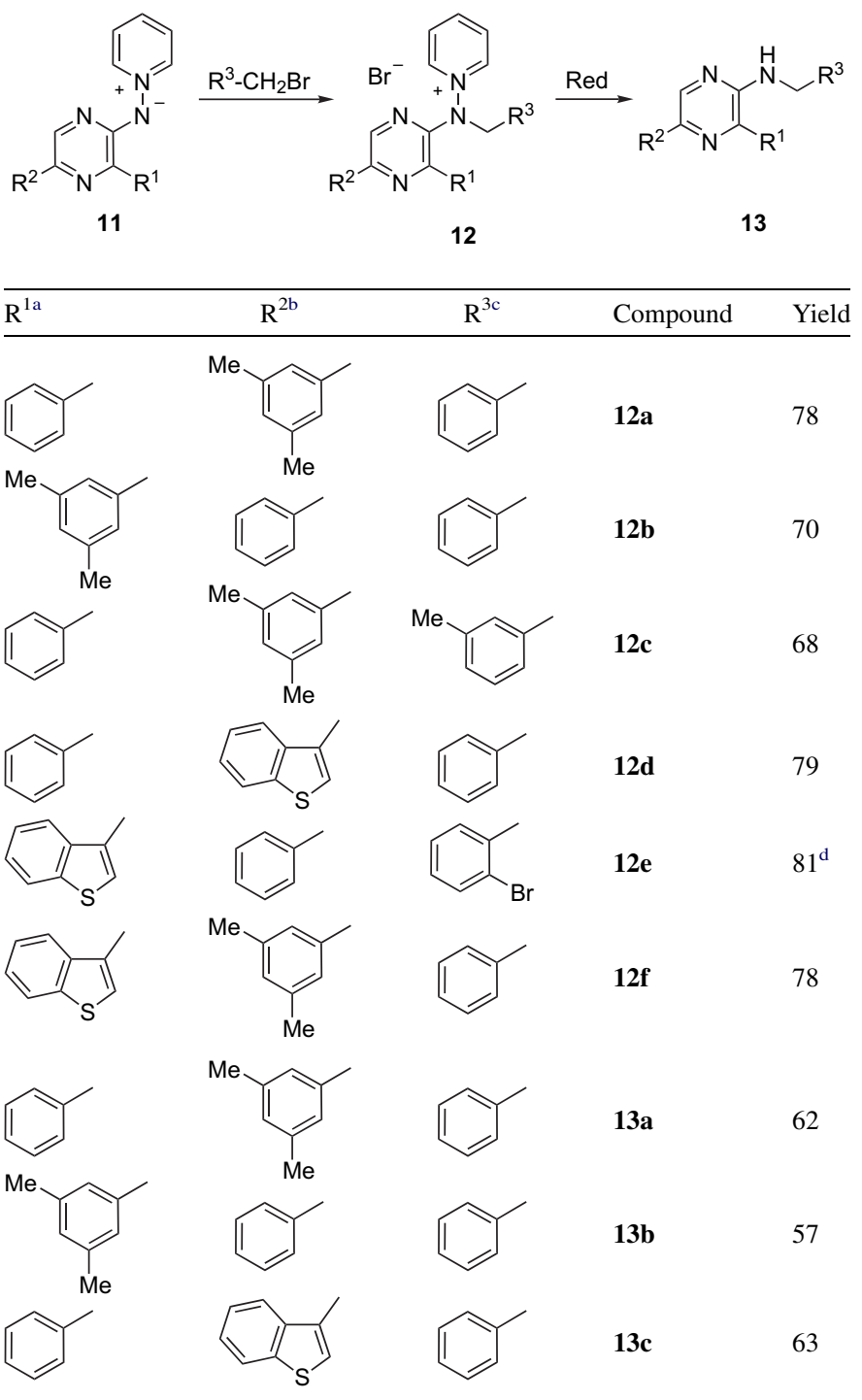

a Positions labelled as (") for NMR analysis.

b Positions labelled as ('"') for NMR analysis.

c Positions labelled as ('"')' for NMR analysis.

${ }^{\mathrm{d}}$ Reaction time: $270 \mathrm{~h}$. $2.34\left(3 \mathrm{H}, \mathrm{s}, \mathrm{CH}_{3}\right) ;{ }^{13} \mathrm{C}$ NMR $\left(75 \mathrm{MHz}, \mathrm{CD}_{3} \mathrm{OD}\right): \delta 164.5$ $\left(C 2^{\prime}\right), 144.5\left(C 6^{\prime}\right), 144.0(C 2(6)), 137.0(C 4), 137.0\left(C 1^{\prime \prime \prime}\right.$ or $\left.C 4^{\prime \prime \prime}\right), 136.9\left(C 1^{\prime \prime \prime}\right.$ or $\left.C 4^{\prime \prime \prime}\right), 136.8\left(C 4^{\prime}\right), 130.5\left(C 3^{\prime \prime \prime}\left(5^{\prime \prime \prime}\right)\right)$, $128.2(C 3(5)), 126.3\left(C 2^{\prime \prime \prime}\left(6^{\prime \prime \prime}\right)\right), 125.5\left(C 5^{\prime}\right), 112.4\left(C 3^{\prime}\right)$, $21.1\left(\mathrm{CH}_{3}\right)$. MS (CI, $\left.\mathrm{m} / \mathrm{z}\right): 262(100, \mathrm{M}+1), 261(40), 185$ (11). Anal. Calcd for $\mathrm{C}_{17} \mathrm{H}_{15} \mathrm{~N}_{3} \cdot 1 / 4 \mathrm{H}_{2} \mathrm{O}: \mathrm{C}, 76.80 ; \mathrm{H}, 5.88$; $\mathrm{N}, 15.81$. Found: C, 76.69; H, 6.15; N, 15.69 .

\subsection{3. $N$-[5'-(3-Hydroxymethylphenyl)pyridin-2-yl]-} pyridinium aminide $(\mathbf{3 c})$

Red oil (263 mg, 95\%), mp hydrobromide $187-188^{\circ} \mathrm{C}$ (yellowish solid, ethanol); IR (KBr) $\nu_{\max }\left(\mathrm{cm}^{-1}\right): 1597,1469,1377$, 1299, 1150, 1000, 790; ${ }^{1} \mathrm{H}$ NMR (300 MHz, $\left.\mathrm{CD}_{3} \mathrm{OD}\right): \delta 8.78$ $(2 \mathrm{H}, \mathrm{dd}, J=7.0$ and $1.2 \mathrm{~Hz}, H 2(6)), 8.03(1 \mathrm{H}, \mathrm{tt}, J=7.7$ and $1.2 \mathrm{~Hz}, H 4), 7.98\left(1 \mathrm{H}\right.$, br d, $\left.J=2.6 \mathrm{~Hz}, H 6^{\prime}\right), 7.82(2 \mathrm{H}, \mathrm{dd}$, $J=7.7$ and $7.0 \mathrm{~Hz}, H 3(5)), 7.72(1 \mathrm{H}, \mathrm{dd}, J=8.9$ and $2.6 \mathrm{~Hz}$, $\left.H 4^{\prime}\right), 7.52\left(1 \mathrm{H}, \mathrm{m}, H 2^{\prime \prime \prime}\right), 7.42(1 \mathrm{H}, \mathrm{dt}, J=7.6$ and $1.7 \mathrm{~Hz}$, $\left.H 6^{\prime \prime \prime}\right), 7.37\left(1 \mathrm{H}\right.$, ap t, $\left.J=7.3 \mathrm{~Hz}, H 5^{\prime \prime \prime}\right), 7.26(1 \mathrm{H}, \mathrm{dt}, J=7.0$ and $\left.1.7 \mathrm{~Hz}, H 4^{\prime \prime \prime}\right), 6.62\left(1 \mathrm{H}, \mathrm{d}, J=8.9 \mathrm{~Hz}, H 3^{\prime}\right), 4.66(2 \mathrm{H}, \mathrm{s}$, $\left.\mathrm{CH}_{2}\right) ;{ }^{13} \mathrm{C}$ NMR $\left(75 \mathrm{MHz}, \mathrm{CD}_{3} \mathrm{OD}\right): \delta 165.0\left(\mathrm{C2}^{\prime}\right), 144.9$ (C2(6)), $144.5\left(C 6^{\prime}\right), 143.3\left(C 3^{\prime \prime \prime}\right), 140.1\left(C 1^{\prime \prime \prime}\right), 137.7(C 4)$, $137.1\left(C 4^{\prime}\right), 129.9\left(C 5^{\prime \prime \prime}\right), 128.5(C 3(5)), 125.9\left(C 4^{\prime \prime \prime}\right), 125.4$ $\left(C 5^{\prime}\right), 125.4\left(C 6^{\prime \prime \prime}\right), 125.0\left(C 2^{\prime \prime \prime}\right), 112.3\left(C 3^{\prime}\right), 65.2\left(C \mathrm{H}_{2}\right)$. MS (CI, m/z): 278 (100, M+1), 277 (47), 260 (25), 201 (65). Anal. Calcd for $\mathrm{C}_{17} \mathrm{H}_{15} \mathrm{~N}_{3} \mathrm{O} \cdot 2 \mathrm{HBr}$ : C, 46.50; H, 3.90; N, 9.57. Found: C, 46.56; H, 3.69; N, 9.38.

\subsubsection{N-[5-(4-Hydroxymethylphenyl)pyridin-2-yl]- pyridinium aminide $(\mathbf{3 d})$ \\ See Ref. 8i.}

\subsubsection{N-[5-(4-Methoxyphenyl)pyridin-2-yl]pyridinium aminide (3e)}

Red solid (325 mg, 91\%, dichloromethane/diethyl ether), mp $151-152^{\circ} \mathrm{C}$; IR $(\mathrm{KBr}) \nu_{\max }\left(\mathrm{cm}^{-1}\right): 1598,1466,1376$, $1279,1248,1133,817,669 ;{ }^{1} \mathrm{H}$ NMR $\left(300 \mathrm{MHz}, \mathrm{CD}_{3} \mathrm{OD}\right)$ :

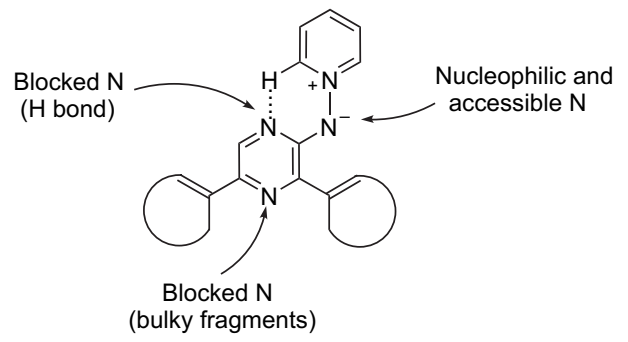

Figure 3. 
$\delta 8.78(2 \mathrm{H}, \mathrm{dd}, J=7.0$ and $1.3 \mathrm{~Hz}, H 2(6)), 7.97(1 \mathrm{H}, \mathrm{tt}, J=7.7$ and $1.3 \mathrm{~Hz}, H 4), 7.92\left(1 \mathrm{H}, \mathrm{dd}, J=2.5\right.$ and $\left.0.6 \mathrm{~Hz}, H 6^{\prime}\right), 7.75$ $(2 \mathrm{H}, \mathrm{dd}, J=7.7$ and $7.0 \mathrm{~Hz}, H 3(5)), 7.64(1 \mathrm{H}, \mathrm{dd}, J=8.8$ and $\left.2.5 \mathrm{~Hz}, H 4^{\prime}\right), 7.41\left(2 \mathrm{H}, \mathrm{d}, J=8.8 \mathrm{~Hz}, H 2^{\prime \prime \prime}\left(6^{\prime \prime \prime}\right)\right), 6.95(2 \mathrm{H}, \mathrm{d}$, $\left.J=8.8 \mathrm{~Hz}, H 3^{\prime \prime \prime}\left(5^{\prime \prime \prime}\right)\right), 6.60\left(1 \mathrm{H}, \mathrm{dd}, J=8.8\right.$ and $\left.0.6 \mathrm{~Hz}, H 3^{\prime}\right)$, $3.81\left(3 \mathrm{H}, \mathrm{s}, \mathrm{CH}_{3}\right) ;{ }^{13} \mathrm{C} \mathrm{NMR}\left(75 \mathrm{MHz}, \mathrm{CD}_{3} \mathrm{OD}\right): \delta 164.4$ $\left(C 2^{\prime}\right), 159.9\left(C 4^{\prime \prime \prime}\right), 144.2\left(C 6^{\prime}\right), 144.2(C 2(6)), 137.2(C 4)$, $136.9\left(C 4^{\prime}\right), 132.5\left(C 1^{\prime \prime \prime}\right), 128.4(C 3(5)), 127.6\left(C 2^{\prime \prime \prime}\left(6^{\prime \prime \prime}\right)\right)$, $125.5\left(C 5^{\prime}\right), 115.3\left(C 3^{\prime \prime \prime}\left(5^{\prime \prime \prime}\right)\right), 112.4\left(C 3^{\prime}\right), 55.7\left(C \mathrm{H}_{3}\right)$. MS (CI, m/z): 278 (100, M+1), 277 (71), 199 (9). Anal. Calcd for $\mathrm{C}_{17} \mathrm{H}_{15} \mathrm{~N}_{3} \mathrm{O} \cdot 1 / 4 \mathrm{H}_{2} \mathrm{O}$ : C, 72.45; H, 5.54; N, 14.91. Found: C, 72.49; H, 5.51; N, 14.98 .

\subsection{6. $N-\{5-[4-(N, N$-Dimethylamino $)$ phenyl]pyridin-2-yl $\}-$ \\ pyridinium aminide (3f)}

Red solid (250 mg, 86\%, ethyl acetate), mp $156-157^{\circ} \mathrm{C}$; IR $(\mathrm{KBr}) \nu_{\max }\left(\mathrm{cm}^{-1}\right): 1612,1598,1471,1381,1302,1138$, 983, 815, 670; ${ }^{1} \mathrm{H}$ NMR (300 MHz, CD $\left.3 \mathrm{OD}\right): \delta 8.79(2 \mathrm{H}$, $\mathrm{dd}, J=7.0$ and $1.3 \mathrm{~Hz}, H 2(6)), 7.96(1 \mathrm{H}, \mathrm{tt}, J=7.7$ and $1.3 \mathrm{~Hz}, H 4), 7.92\left(1 \mathrm{H}, \mathrm{dd}, J=2.6\right.$ and $\left.0.7 \mathrm{~Hz}, H 6^{\prime}\right), 7.77$ $(2 \mathrm{H}, \mathrm{dd}, J=7.7$ and $7.0 \mathrm{~Hz}, H 3(5)), 7.66(1 \mathrm{H}, \mathrm{dd}, J=8.9$ and $\left.2.6 \mathrm{~Hz}, H 4^{\prime}\right), 7.37\left(2 \mathrm{H}, \mathrm{d}, J=9.0 \mathrm{~Hz}, H 2^{\prime \prime \prime}\left(6^{\prime \prime \prime}\right)\right), 6.84(2 \mathrm{H}, \mathrm{d}$, $\left.J=9.0 \mathrm{~Hz}, H 3^{\prime \prime \prime}\left(5^{\prime \prime \prime}\right)\right), 6.61\left(1 \mathrm{H}, \mathrm{dd}, J=8.9\right.$ and $\left.0.7 \mathrm{~Hz}, H 3^{\prime}\right)$, $2.95\left(6 \mathrm{H}, \mathrm{s}, \mathrm{CH}_{3}\right) ;{ }^{13} \mathrm{C} \mathrm{NMR}\left(75 \mathrm{MHz}, \mathrm{CD}_{3} \mathrm{OD}\right): \delta 164.1$ $\left(C 2^{\prime}\right), 151.0\left(C 4^{\prime \prime \prime}\right), 144.0(C 2(6)), 143.7\left(C 6^{\prime}\right), 136.9\left(C 4^{\prime}\right)$, $136.7(C 4), 128.6\left(C 1^{\prime \prime \prime}\right), 128.4(C 3(5)), 127.1\left(C 2^{\prime \prime \prime}\left(6^{\prime \prime \prime}\right)\right)$, $126.2\left(C 5^{\prime}\right), 114.6\left(C 3^{\prime \prime \prime}\left(5^{\prime \prime \prime}\right)\right), 112.5\left(C 3^{\prime}\right), 41.1\left(C \mathrm{H}_{3}\right) . \mathrm{MS}$ (CI, m/z): 291 (100, M+1), 290 (56), 214 (55), 108 (8), 80 (29). Anal. Calcd for $\mathrm{C}_{18} \mathrm{H}_{18} \mathrm{~N}_{4} \cdot 1 / 4 \mathrm{H}_{2} \mathrm{O}$ : C, 73.31; H, 6.33; N, 19.01. Found: C, 73.60; H, 6.35; N, 18.89 .

\subsection{7. $N$-[5-(3-Chlorophenyl)pyridin-2-yl]pyridinium aminide (3g)}

Orange oil (251 mg, 89\%), mp hydrobromide $219-220{ }^{\circ} \mathrm{C}$ (yellowish solid, ethanol); IR (KBr) $\nu_{\max }\left(\mathrm{cm}^{-1}\right): 1590,1464$, 1385, 1121, 694; ${ }^{1} \mathrm{H}$ NMR (500 MHz, CD $\left.3 \mathrm{OD}\right): \delta 8.77$ $(2 \mathrm{H}, \mathrm{dd}, J=6.9$ and $1.4 \mathrm{~Hz}, H 2(6)), 7.99(1 \mathrm{H}, \mathrm{tt}, J=7.7$ and $1.4 \mathrm{~Hz}, H 4), 7.95\left(1 \mathrm{H}, \mathrm{d}, J=2.6 \mathrm{~Hz}, H 6^{\prime}\right), 7.78(2 \mathrm{H}, \mathrm{dd}$, $J=7.7$ and $6.9 \mathrm{~Hz}, H 3(5)), 7.63(1 \mathrm{H}, \mathrm{dd}, J=8.9$ and $2.6 \mathrm{~Hz}$, $\left.H 4^{\prime}\right), 7.48\left(1 \mathrm{H}, \mathrm{t}, J=1.9 \mathrm{~Hz}, H 2^{\prime \prime \prime}\right), 7.39(1 \mathrm{H}$, ap dt, $J=7.8$ and $\left.1.5 \mathrm{~Hz}, H 6^{\prime \prime \prime}\right), 7.31\left(1 \mathrm{H}, \mathrm{t}, J=7.8 \mathrm{~Hz}, H 5^{\prime \prime \prime}\right), 7.21(1 \mathrm{H}$, $\left.\mathrm{m}, H 4^{\prime \prime \prime}\right), 6.59\left(1 \mathrm{H}, \mathrm{d}, J=8.9 \mathrm{~Hz}, H 3^{\prime}\right) ;{ }^{13} \mathrm{C} \mathrm{NMR}(125 \mathrm{MHz}$, $\left.\mathrm{CD}_{3} \mathrm{OD}\right): \delta 165.2\left(C 2^{\prime}\right), 145.2\left(C 6^{\prime}\right), 144.4(C 2(6)), 142.1$ $\left(C 1^{\prime \prime \prime}\right), 137.6(C 4), 136.6\left(C 4^{\prime}\right), 135.7\left(C 3^{\prime \prime \prime}\right), 131.3\left(C 5^{\prime \prime \prime}\right)$, $128.4(C 3(5)), 126.9\left(C 4^{\prime \prime \prime}\right), 126.1\left(C 2^{\prime \prime \prime}\right), 124.6\left(C 6^{\prime \prime \prime}\right)$, $123.6\left(C 5^{\prime}\right), 112.3\left(C 3^{\prime}\right)$. MS (CI, m/z): 284/282 (37/100, M+1), 283/281 (44/63), 246 (24), 205 (10). Anal. Calcd for $\mathrm{C}_{16} \mathrm{H}_{12} \mathrm{ClN}_{3} \cdot \mathrm{HBr} \cdot 1 / 4 \mathrm{H}_{2} \mathrm{O}$ : C, 52.34; H, 3.71; N, 11.44. Found: C, 52.31; H, 3.79; N, 11.46.

\subsubsection{N-[5-(4-Formylphenyl)pyridin-2-yl]pyridinium aminide (3h)}

Orange solid (262 mg, 95\%, ethanol/ethyl acetate), mp 163$164{ }^{\circ} \mathrm{C}$; IR $(\mathrm{KBr}) \nu_{\max }\left(\mathrm{cm}^{-1}\right): 1691,1584,1470,1393,1305$, 1166, 769; ${ }^{1} \mathrm{H}$ NMR (300 MHz, CD $\left.{ }_{3} \mathrm{OD}\right): \delta 9.97(1 \mathrm{H}, \mathrm{s}$, CHO), 8.80 (2H, dd, $J=6.8$ and $1.3 \mathrm{~Hz}, H 2(6)), 8.12(1 \mathrm{H}, \mathrm{m}$, $H 4), 8.10\left(1 \mathrm{H}, \mathrm{m}, H 6^{\prime}\right), 7.93\left(2 \mathrm{H}, \mathrm{d}, J=8.4 \mathrm{~Hz}, H 3^{\prime \prime \prime}\left(5^{\prime \prime \prime}\right)\right)$,
$7.88(2 \mathrm{H}, \mathrm{dd}, J=7.7$ and $6.8 \mathrm{~Hz}, H 3(5)), 7.80(1 \mathrm{H}, \mathrm{dd}, J=8.9$ and $\left.2.6 \mathrm{~Hz}, H 4^{\prime}\right), 7.74\left(2 \mathrm{H}, \mathrm{d}, J=8.4 \mathrm{~Hz}, H 2^{\prime \prime \prime}\left(6^{\prime \prime \prime}\right)\right), 6.63$ $\left(1 \mathrm{H}, \mathrm{d}, J=8.9 \mathrm{~Hz}, H 3^{\prime}\right) ;{ }^{13} \mathrm{C} \mathrm{NMR}\left(75 \mathrm{MHz}, \mathrm{CD}_{3} \mathrm{OD}\right)$ : $\delta 193.6(C \mathrm{O}), 165.7\left(C 2^{\prime}\right), 146.4\left(C 1^{\prime \prime \prime}\right), 146.1\left(C 6^{\prime}\right), 145.0$ $(C 2(6)), \quad 138.6(C 4), \quad 136.7 \quad\left(C 4^{\prime}\right), \quad 135.6 \quad\left(C 4^{\prime \prime \prime}\right), \quad 131.5$ $\left(C 3^{\prime \prime \prime}\left(5^{\prime \prime \prime}\right)\right), 128.7(C 3(5)), 126.3\left(C 2^{\prime \prime \prime}\left(6^{\prime \prime \prime}\right)\right), 123.2\left(C 5^{\prime}\right)$, $112.3\left(C 3^{\prime}\right)$. MS (CI, m/z): $276(100, \mathrm{M}+1), 275$ (29), 199 (17). Anal. Calcd for $\mathrm{C}_{17} \mathrm{H}_{13} \mathrm{~N}_{3} \mathrm{O} \cdot 1 / 4 \mathrm{H}_{2} \mathrm{O}: \mathrm{C}, 72.96 ; \mathrm{H}, 4.87$; N, 15.02. Found: C, 72.96; H, 5.17; N, 15.04.

\subsubsection{N-[5-(4-Acetylphenyl)pyridin-2-yl]pyridinium aminide (3i)}

Orange solid (217 mg, 75\%, dichloromethane), mp 188$189{ }^{\circ} \mathrm{C}$; IR $(\mathrm{KBr}) \nu_{\max }\left(\mathrm{cm}^{-1}\right): 1671,1582,1458,1374,1267$, 1144, 818; ${ }^{1} \mathrm{H}$ NMR $\left(300 \mathrm{MHz}, \mathrm{CD}_{3} \mathrm{OD}\right): \delta 8.80(2 \mathrm{H}, \mathrm{dd}$, $J=7.0$ and $1.2 \mathrm{~Hz}, H 2(6)), 8.11(1 \mathrm{H}, \mathrm{tt}, J=7.7$ and $1.2 \mathrm{~Hz}$, $H 4), 8.10\left(1 \mathrm{H}, \mathrm{dd}, J=2.6\right.$ and $\left.0.8 \mathrm{~Hz}, H 6^{\prime}\right), 8.03(2 \mathrm{H}, \mathrm{d}$, $\left.J=8.6 \mathrm{~Hz}, H 3^{\prime \prime \prime}\left(5^{\prime \prime \prime}\right)\right), 7.87(2 \mathrm{H}, \mathrm{dd}, J=7.7$ and $7.0 \mathrm{~Hz}, H 3(5))$, $7.79\left(1 \mathrm{H}\right.$, dd, $J=8.9$ and $\left.2.6 \mathrm{~Hz}, H 4^{\prime}\right), 7.67(2 \mathrm{H}, \mathrm{d}, J=8.6 \mathrm{~Hz}$, $\left.H 2^{\prime \prime \prime}\left(6^{\prime \prime \prime}\right)\right), 6.63\left(1 \mathrm{H}, \mathrm{dd}, J=8.9\right.$ and $\left.0.8 \mathrm{~Hz}, H 3^{\prime}\right), 2.63(3 \mathrm{H}, \mathrm{s}$, $\left.\mathrm{CH}_{3}\right) ;{ }^{13} \mathrm{C} \mathrm{NMR}\left(75 \mathrm{MHz}, \mathrm{CD}_{3} \mathrm{OD}\right): \delta 200.2$ (CO), 165.7 $\left(C 2^{\prime}\right), 145.8\left(C 6^{\prime}\right), 145.1\left(C 1^{\prime \prime \prime}\right), 145.0(C 2(6)), 138.5(C 4)$, $136.7\left(C 4^{\prime}\right), 135.7\left(C 4^{\prime \prime \prime}\right), 130.3\left(C 3^{\prime \prime \prime}\left(5^{\prime \prime \prime}\right)\right), 128.7(C 3(5))$, $126.0\left(C 2^{\prime \prime \prime}\left(6^{\prime \prime \prime}\right)\right), 123.5\left(C 5^{\prime}\right), 112.3\left(C 3^{\prime}\right), 26.5\left(C \mathrm{H}_{3}\right)$. Anal. Calcd for $\mathrm{C}_{18} \mathrm{H}_{15} \mathrm{~N}_{3} \mathrm{O}$ : C, 74.72; H, 5.23; N, 14.52. Found: C, 74.69; H, 5.42; N, 14.49 .

\subsubsection{N-[5-(trans-2-Phenylvinyl)pyridin-2-yl]pyridinium aminide (3j)}

Red solid (191 mg, 70\%, ethyl acetate/hexane), mp 159$160{ }^{\circ} \mathrm{C}$; IR $(\mathrm{KBr}) \nu_{\max }\left(\mathrm{cm}^{-1}\right): 1589,1483,1468,1386$, 1345, 1156, 958, 755; ${ }^{1} \mathrm{H}$ NMR (300 MHz, $\left.\mathrm{CD}_{3} \mathrm{OD}\right): \delta 8.75$ $(2 \mathrm{H}, \mathrm{dd}, J=7.0$ and $1.2 \mathrm{~Hz}, H 2(6)), 8.03(1 \mathrm{H}, \mathrm{tt}, J=7.8$ and $1.2 \mathrm{~Hz}, H 4), 7.80(2 \mathrm{H}, \mathrm{dd}, J=7.8$ and $7.0 \mathrm{~Hz}, H 3(5)), 7.76$ $\left(1 \mathrm{H}, \mathrm{m}, H 6^{\prime}\right), 7.74\left(1 \mathrm{H}, \mathrm{m}, H 4^{\prime}\right), 7.49\left(2 \mathrm{H}, \mathrm{m}, H 2^{\prime \prime \prime}\left(6^{\prime \prime \prime}\right)\right)$, $7.32\left(2 \mathrm{H}\right.$, ap t, $\left.J=7.6 \mathrm{~Hz}, H 3^{\prime \prime \prime}\left(5^{\prime \prime \prime}\right)\right), 7.19(1 \mathrm{H}, \mathrm{tt}, J=7.3$ and $\left.1.3 \mathrm{~Hz}, H 4^{\prime \prime \prime}\right), 7.01\left(1 \mathrm{H}\right.$, ap d, $\left.J=16.4 \mathrm{~Hz}, H_{\alpha}\right), 6.88(1 \mathrm{H}$, ap $\left.\mathrm{d}, J=16.4 \mathrm{~Hz}, H_{\beta}\right), 6.57\left(1 \mathrm{H}, \mathrm{dd}, J=8.4\right.$ and $\left.1.4 \mathrm{~Hz}, H 3^{\prime}\right)$; ${ }^{13} \mathrm{C}$ NMR (75 MHz, CD $\left.\mathrm{OD}\right): \delta 165.1\left(C 2^{\prime}\right), 147.0\left(C 6^{\prime}\right)$, $144.6(C 2(6)), 139.5\left(C 1^{\prime \prime \prime}\right), 137.9(C 4), 134.8\left(C 4^{\prime}\right), 129.6$ $\left(C 3^{\prime \prime \prime}\left(5^{\prime \prime \prime}\right)\right), 128.5(C 3(5)), 127.7\left(C 4^{\prime \prime \prime}\right), 126.9\left(C 2^{\prime \prime \prime}\left(6^{\prime \prime \prime}\right)\right)$, $126.8\left(C_{\alpha}\right), 124.9\left(C_{\beta}\right), 122.6\left(C 5^{\prime}\right), 112.8\left(C 3^{\prime}\right) . \mathrm{MS}(\mathrm{CI}$, $\mathrm{m} / z): 274$ (100, M+1), 273 (48), 197 (31). Anal. Calcd for $\mathrm{C}_{18} \mathrm{H}_{15} \mathrm{~N}_{3} \cdot 1 / 4 \mathrm{H}_{2} \mathrm{O}$ : C, 78.24; H, 5.59; N, 15.21. Found: C, 78.31; H, 5.72; N, 15.21.

\subsubsection{N-[5-(Benzo[b]furan-2-yl)pyridin-2-yl]pyridinium aminide (3k)}

Orange solid (256 mg, 89\%, ethyl acetate/hexane), mp $141-142^{\circ} \mathrm{C}$; IR $(\mathrm{KBr}) \nu_{\max }\left(\mathrm{cm}^{-1}\right): 1619,1487,1451$, 1395, 1137, 799, 744, 669; ${ }^{1} \mathrm{H}$ NMR (300 MHz, $\left.\mathrm{CD}_{3} \mathrm{OD}\right)$ : $\delta 8.75(2 \mathrm{H}$, dd, $J=6.9$ and $1.3 \mathrm{~Hz}, H 2(6)), 8.22(1 \mathrm{H}$, br d, $\left.J=2.1 \mathrm{~Hz}, H 6^{\prime}\right), 8.08(1 \mathrm{H}, \mathrm{tt}, J=7.7$ and $1.3 \mathrm{~Hz}, H 4), 7.84$ $(2 \mathrm{H}, \mathrm{dd}, J=7.7$ and $6.9 \mathrm{~Hz}, H 3(5)), 7.81(1 \mathrm{H}, \mathrm{dd}, J=8.9$ and $\left.2.4 \mathrm{~Hz}, H 4^{\prime}\right), 7.51\left(1 \mathrm{H}, \mathrm{m}, H 4^{\prime \prime \prime}\right), 7.44\left(1 \mathrm{H}, \mathrm{m}, H 7^{\prime \prime \prime}\right), 7.19$ $\left(2 \mathrm{H}, \mathrm{m}, H 5^{\prime \prime \prime}\right.$ and $\left.H 6^{\prime \prime \prime}\right), 6.84\left(1 \mathrm{H}, \mathrm{d}, J=0.9 \mathrm{~Hz}, H 3^{\prime \prime \prime}\right), 6.59$ $\left(1 \mathrm{H}, \mathrm{dd}, J=8.9\right.$ and $\left.0.6 \mathrm{~Hz}, H 3^{\prime}\right) ;{ }^{13} \mathrm{C} \mathrm{NMR}(75 \mathrm{MHz}$, 
$\left.\mathrm{CD}_{3} \mathrm{OD}\right): \delta 165.1\left(C 2^{\prime}\right), 156.1\left(C 2^{\prime \prime \prime}\right), 155.3\left(C 7^{\prime \prime \prime} \mathrm{a}\right), 144.6$ $(C 2(6)), 143.8\left(C 6^{\prime}\right), 138.2(C 4), 134.5\left(C 4^{\prime}\right), 130.7\left(C 3^{\prime \prime \prime} \mathrm{a}\right)$, $128.3(C 3(5)), 124.1\left(C 5^{\prime \prime \prime}\right.$ or $\left.C 6^{\prime \prime \prime}\right), 123.6\left(C 5^{\prime \prime \prime}\right.$ or $\left.C 6^{\prime \prime \prime}\right)$, 120.9 (C4'"'), $115.1\left(C 5^{\prime}\right), 111.9\left(C 3^{\prime}\right), 111.2\left(C 7^{\prime \prime \prime}\right), 98.6$ $\left(\mathrm{C}^{\prime \prime \prime}\right)$. MS (CI, $\left.\mathrm{m} / \mathrm{z}\right): 288(100, \mathrm{M}+1), 211(38), 108(10)$, 80 (60). Anal. Calcd for $\mathrm{C}_{18} \mathrm{H}_{13} \mathrm{~N}_{3} \mathrm{O} \cdot 1 / 4 \mathrm{H}_{2} \mathrm{O}: \mathrm{C}, 74.01 ; \mathrm{H}$, 4.66; N, 14.41. Found: C, 74.24; H, 4.83; N, 14.43.

\subsubsection{N-[5-(Pyridin-3-yl)pyridin-2-yl]pyridinium}

aminide $(3 \mathrm{l})$

Yellow oil $(236 \mathrm{mg}, 95 \%)$; IR $(\mathrm{KBr}) \nu_{\max }\left(\mathrm{cm}^{-1}\right)$ : 1598 , $1462,1417,1376,1300,1147,1019,804 ;{ }^{1} \mathrm{H}$ NMR $\left(500 \mathrm{MHz}, \mathrm{CD}_{3} \mathrm{OD}\right): \delta 8.80(2 \mathrm{H}, \mathrm{dd}, J=7.0$ and $1.2 \mathrm{~Hz}$, $H 2(6)), 8.70\left(1 \mathrm{H}, \mathrm{dd}, J=2.4\right.$ and $\left.0.7 \mathrm{~Hz}, H 2^{\prime \prime \prime}\right), 8.41(1 \mathrm{H}$, dd, $J=4.8$ and $\left.1.5 \mathrm{~Hz}, H 6^{\prime \prime \prime}\right), 8.09(1 \mathrm{H}, \mathrm{tt}, J=7.7$ and $1.2 \mathrm{~Hz}$, $H 4), 8.00\left(1 \mathrm{H}, \mathrm{dd}, J=2.5\right.$ and $\left.0.7 \mathrm{~Hz}, H 6^{\prime}\right), 7.98(1 \mathrm{H}$, ddd, $J=8.0,2.4$ and $\left.1.5 \mathrm{~Hz}, H 4^{\prime \prime \prime}\right), 7.86(2 \mathrm{H}, \mathrm{dd}, J=7.7$ and $7.0 \mathrm{~Hz}, H 3(5)), 7.73\left(1 \mathrm{H}, \mathrm{dd}, J=8.9\right.$ and $\left.2.5 \mathrm{~Hz}, H 4^{\prime}\right), 7.45$ $\left(1 \mathrm{H}\right.$, ddd, $J=8.0,4.8$ and $\left.0.7 \mathrm{~Hz}, H 5^{\prime \prime \prime}\right), 6.64(1 \mathrm{H}, \mathrm{dd}, J=8.9$ and $\left.0.7 \mathrm{~Hz}, \mathrm{H3}^{\prime}\right) ;{ }^{13} \mathrm{C}$ NMR $\left(125 \mathrm{MHz}, \mathrm{CD}_{3} \mathrm{OD}\right): \delta 165.7$ $\left(C 2^{\prime}\right), 147.4\left(C 6^{\prime \prime \prime}\right), 146.8\left(C 2^{\prime \prime \prime}\right), 145.5\left(C 6^{\prime}\right), 144.9(C 2(6))$, $138.3(C 4), 136.6\left(C 3^{\prime \prime \prime}\right), 136.6\left(C 4^{\prime}\right), 134.6\left(C 4^{\prime \prime \prime}\right), 128.6$ $(C 3(5)), 125.4\left(C 5^{\prime \prime \prime}\right), 121.1\left(C 5^{\prime}\right), 112.4\left(C 3^{\prime}\right)$. HRMS (FAB) calcd for $\mathrm{C}_{15} \mathrm{H}_{13} \mathrm{~N}_{4}[\mathrm{M}+\mathrm{H}]^{+}$249.1140, found 249.1149 .

\subsubsection{N-(5-Phenylpyrazin-2-yl)pyridinium aminide (4a)}

Yellow solid $(228 \mathrm{mg}, 90 \%$, dichloromethane/diethyl ether), mp $171-172{ }^{\circ} \mathrm{C}$; IR $(\mathrm{KBr}) \nu_{\max }\left(\mathrm{cm}^{-1}\right): 1514,1488$, 1466, 1385, 1301, 1147, 992, 474; ${ }^{1} \mathrm{H}$ NMR $(300 \mathrm{MHz}$, $\left.\mathrm{CD}_{3} \mathrm{OD}\right): \delta 8.87(2 \mathrm{H}, \mathrm{dd}, J=7.0$ and $1.3 \mathrm{~Hz}, H 2(6)), 8.14$ $(1 \mathrm{H}, \mathrm{tt}, J=7.7$ and $1.3 \mathrm{~Hz}, H 4), 8.08\left(1 \mathrm{H}, \mathrm{d}, J=1.5 \mathrm{~Hz}, H 6^{\prime}\right)$, $7.96\left(1 \mathrm{H}, \mathrm{d}, J=1.5 \mathrm{~Hz}, H 3^{\prime}\right), 7.89(2 \mathrm{H}, \mathrm{dd}, J=7.7$ and $7.0 \mathrm{~Hz}$, $H 3(5)), 7.78\left(2 \mathrm{H}\right.$, ap dd, $J=8.5$ and $\left.1.3 \mathrm{~Hz}, H 2^{\prime \prime \prime}\left(6^{\prime \prime \prime}\right)\right), 7.42$ $\left(2 \mathrm{H}\right.$, ap dd, $J=8.5$ and $\left.7.4 \mathrm{~Hz}, H 3^{\prime \prime \prime}\left(5^{\prime \prime \prime}\right)\right), 7.30(1 \mathrm{H}, \mathrm{tt}, J=7.4$ and $\left.1.3 \mathrm{~Hz}, H 4^{\prime \prime \prime}\right) ;{ }^{13} \mathrm{C}$ NMR $\left(75 \mathrm{MHz}, \mathrm{CD}_{3} \mathrm{OD}\right): \delta 160.9$ $\left(C 2^{\prime}\right), 144.8(C 2(6)), 139.4\left(C 5^{\prime}\right), 139.2\left(C 1^{\prime \prime \prime}\right), 139.0(C 4)$, $138.7\left(C 6^{\prime}\right), 136.5\left(C 3^{\prime}\right), 129.7\left(C 3^{\prime \prime \prime}\left(5^{\prime \prime \prime}\right)\right), 128.6(C 3(5))$, $128.2\left(C 4^{\prime \prime \prime}\right), 125.9\left(C 2^{\prime \prime \prime}\left(6^{\prime \prime \prime}\right)\right)$. Anal. Calcd for $\mathrm{C}_{15} \mathrm{H}_{12} \mathrm{~N}_{4}$ : C, 72.56; H, 4.87; N, 22.57. Found: C, 72.27; H, 5.03; N, 22.47 .

\subsubsection{N-[5-(4-Methoxyphenyl)pyrazin-2-yl]pyridinium aminide $(4 \mathrm{~b})$}

Orange solid ( $242 \mathrm{mg}, 87 \%$, ethanol), mp $145-146{ }^{\circ} \mathrm{C}$; IR $(\mathrm{KBr}) \nu_{\max }\left(\mathrm{cm}^{-1}\right): 1606,1568,1491,1374,1236,1147,992$, $669 ;{ }^{1} \mathrm{H}$ NMR $\left(300 \mathrm{MHz}, \mathrm{CD}_{3} \mathrm{OD}\right): \delta 8.86(2 \mathrm{H}, \mathrm{dd}, J=7.0$ and $1.3 \mathrm{~Hz}, H 2(6)), 8.10(1 \mathrm{H}, \mathrm{tt}, J=7.6$ and $1.3 \mathrm{~Hz}, H 4), 8.01(1 \mathrm{H}$, d, $\left.J=1.6 \mathrm{~Hz}, H 6^{\prime}\right), 7.92\left(1 \mathrm{H}, \mathrm{d}, J=1.6 \mathrm{~Hz}, H 3^{\prime}\right), 7.86(2 \mathrm{H}, \mathrm{dd}$, $J=7.6$ and $7.0 \mathrm{~Hz}, H 3(5)), 7.69\left(2 \mathrm{H}, \mathrm{d}, J=8.8 \mathrm{~Hz}, H 2^{\prime \prime \prime}\left(6^{\prime \prime \prime}\right)\right)$, $6.97\left(2 \mathrm{H}, \mathrm{d}, J=8.8 \mathrm{~Hz}, \mathrm{H3}^{\prime \prime \prime}\left(5^{\prime \prime \prime}\right)\right), 3.84\left(3 \mathrm{H}, \mathrm{s}, \mathrm{CH}_{3}\right) ;{ }^{13} \mathrm{C}$ NMR (75 MHz, $\left.\mathrm{CD}_{3} \mathrm{OD}\right): \delta 160.2\left(C 4^{\prime \prime \prime}\right), 159.9\left(C 2^{\prime}\right), 144.1$ $(C 2(6)), 139.1\left(C 5^{\prime}\right), 138.2(C 4), 137.6\left(C 6^{\prime}\right), 135.9\left(C 3^{\prime}\right)$, $131.2\left(C 1^{\prime \prime \prime}\right), \quad 128.2 \quad(C 3(5)), \quad 126.9 \quad\left(C 2^{\prime \prime \prime}\left(6^{\prime \prime \prime}\right)\right), \quad 114.9$ $\left(C 3^{\prime \prime \prime}\left(5^{\prime \prime \prime}\right)\right)$, $55.7\left(\mathrm{CH}_{3}\right)$. Anal. Calcd for $\mathrm{C}_{16} \mathrm{H}_{14} \mathrm{~N}_{4} \mathrm{O}: \mathrm{C}$, 69.05; H, 5.07; N, 20.13. Found: C, 68.69; H, 5.09; N, 20.00.
5.2.15. N-[5-(4-Acetylphenyl)pyrazin-2-yl]pyridinium aminide $(4 \mathrm{c})$

Orange solid (244 mg, 84\%), mp hydrobromide $210-211^{\circ} \mathrm{C}$ (yellowish solid, ethanol/dichloromethane); IR (KBr) $\nu_{\max }$ $\left(\mathrm{cm}^{-1}\right): 1672,1601,1578,1521,1495,1467,1400,1263$, 1146, 994, 828, 666; ${ }^{1} \mathrm{H}$ NMR (300 MHz, $\left.\mathrm{CD}_{3} \mathrm{OD}\right): \delta 8.86$ $(2 \mathrm{H}, \mathrm{dd}, J=6.9$ and $1.3 \mathrm{~Hz}, \mathrm{H} 2(6)), 8.20(1 \mathrm{H}, \mathrm{d}, J=$ $\left.1.5 \mathrm{~Hz}, \mathrm{H6}^{\prime}\right), 8.19(1 \mathrm{H}, \mathrm{tt}, J=8.0$ and $1.3 \mathrm{~Hz}, \mathrm{H} 4), 8.05(2 \mathrm{H}$, d, $\left.J=8.5 \mathrm{~Hz}, \mathrm{H} 2^{\prime \prime \prime}\left(6^{\prime \prime \prime}\right)\right), 7.99\left(1 \mathrm{H}, \mathrm{d}, J=1.5 \mathrm{~Hz}, H 3^{\prime}\right), 7.96$ $\left(2 \mathrm{H}, \mathrm{d}, J=8.5 \mathrm{~Hz}, H 3^{\prime \prime \prime}\left(5^{\prime \prime \prime}\right)\right), 7.93(2 \mathrm{H}, \mathrm{dd}, J=8.0$ and $6.9 \mathrm{~Hz}$, H3(5)), $2.65\left(3 \mathrm{H}, \mathrm{s}, \mathrm{CH}_{3}\right) ;{ }^{13} \mathrm{C}$ NMR $\left(75 \mathrm{MHz}, \mathrm{CD}_{3} \mathrm{OD}\right)$ : $\delta 199.5(C O), 160.8\left(C 2^{\prime}\right), 144.6(C 2(6)), 143.5\left(C 1^{\prime \prime \prime}\right), 139.4$ (C4), $139.2\left(C 6^{\prime}\right), 136.9\left(C 5^{\prime}\right), 136.6\left(C 3^{\prime}\right), 136.1\left(C 4^{\prime \prime \prime}\right), 129.8$ $\left(C 3^{\prime \prime \prime}\left(5^{\prime \prime \prime}\right)\right), 128.4(C 3(5)), 125.1\left(C 2^{\prime \prime \prime}\left(6^{\prime \prime \prime}\right)\right), 26.7\left(C_{3}\right) . M S$ (CI, m/z): 291(100, M+1), 214(89), 108 (20), 80 (97). Anal. Calcd for $\mathrm{C}_{17} \mathrm{H}_{14} \mathrm{~N}_{4} \mathrm{O} \cdot 1 / 4 \mathrm{H}_{2} \mathrm{O}: \mathrm{C}, 69.24 ; \mathrm{H}, 4.96 ; \mathrm{N}, 19.01$. Found: C, 69.44; H, 4.80; N, 19.17 .

\subsubsection{6. $N$-[5-(trans-2-Phenylvinyl)pyrazin-2-yl]pyridinium aminide (4d)}

Red solid (250 mg, 91\%, ethanol), mp $180-181{ }^{\circ} \mathrm{C}$; IR (KBr) $\nu_{\max }\left(\mathrm{cm}^{-1}\right): 1568,1501,1396,1146,1004,748 ;{ }^{1} \mathrm{H}$ NMR (300 MHz, $\left.\mathrm{CD}_{3} \mathrm{OD}\right): \delta 8.84(2 \mathrm{H}, \mathrm{dd}, J=7.0$ and $1.3 \mathrm{~Hz}$, $H 2(6)), 8.13(1 \mathrm{H}, \mathrm{tt}, J=7.7$ and $1.3 \mathrm{~Hz}, H 4), 7.90(1 \mathrm{H}, \mathrm{d}$, $\left.J=1.4 \mathrm{~Hz}, H 6^{\prime}\right), 7.88(2 \mathrm{H}, \mathrm{dd}, J=7.7$ and $7.0 \mathrm{~Hz}, H 3(5)), 7.72$ $\left(1 \mathrm{H}, \mathrm{d}, J=1.4 \mathrm{~Hz}, H 3^{\prime}\right), 7.52(2 \mathrm{H}$, ap dd, $J=8.6$ and $1.3 \mathrm{~Hz}$, $\left.H 2^{\prime \prime \prime}\left(6^{\prime \prime \prime}\right)\right), 7.34\left(2 \mathrm{H}\right.$, ap dd, $J=8.6$ and $\left.7.3 \mathrm{~Hz}, H 3^{\prime \prime \prime}\left(5^{\prime \prime \prime}\right)\right), 7.23$ $\left(1 \mathrm{H}, \mathrm{d}, J=16.1 \mathrm{~Hz}, H_{\beta}\right), 7.22\left(1 \mathrm{H}, \mathrm{tt}, J=7.3\right.$ and $\left.1.3 \mathrm{~Hz}, H 4^{\prime \prime \prime}\right)$, $7.04\left(1 \mathrm{H}, \mathrm{d}, J=16.1 \mathrm{~Hz}, H_{\alpha}\right) ;{ }^{13} \mathrm{C}$ NMR $\left(75 \mathrm{MHz}, \mathrm{CD}_{3} \mathrm{OD}\right)$ : $\delta 160.3\left(C 2^{\prime}\right), 144.3(C 2(6)), 140.6\left(C 3^{\prime}\right), 139.1\left(C 5^{\prime}\right), 138.7$ (C4), $137.5\left(C 1^{\prime \prime \prime}\right), 137.0\left(C 6^{\prime}\right), 129.3\left(C 3^{\prime \prime \prime}\left(5^{\prime \prime \prime}\right)\right), 128.3$ (C3(5)), $127.9\left(C 4^{\prime \prime \prime}\right), 127.0\left(C 2^{\prime \prime \prime}\left(6^{\prime \prime \prime}\right)\right), 127.0\left(C_{\beta}\right), 125.4$ $\left(C_{\alpha}\right)$. Anal. Calcd for $\mathrm{C}_{17} \mathrm{H}_{14} \mathrm{~N}_{4}: \mathrm{C}, 74.43 ; \mathrm{H}, 5.14 ; \mathrm{N}, 20.42$. Found: C, 74.16; H, 5.28; N, 20.21.

\subsubsection{N-[5-(Pyridin-3-yl)pyrazin-2-yl]pyridinium aminide $(\mathbf{4 e})$}

Orange solid (217 mg, 89\%, ethanol), mp $142-143{ }^{\circ} \mathrm{C}$; IR (KBr) $\nu_{\max }\left(\mathrm{cm}^{-1}\right): 1579,1510,1481,1463,1414,1385$, 1147,$996 ;{ }^{1} \mathrm{H}$ NMR $\left(300 \mathrm{MHz}, \mathrm{CD}_{3} \mathrm{OD}\right): \delta 8.98(1 \mathrm{H}, \mathrm{dd}, J=$ 2.3 and $\left.0.7 \mathrm{~Hz}, H 2^{\prime \prime \prime}\right), 8.85(2 \mathrm{H}, \mathrm{dd}, J=7.0$ and $1.3 \mathrm{~Hz}, H 2(6))$, $8.43\left(1 \mathrm{H}, \mathrm{dd}, J=4.8\right.$ and $\left.1.6 \mathrm{~Hz}, H 6^{\prime \prime \prime}\right), 8.23(1 \mathrm{H}$, ddd, $J=8.1$, 2.3 and $\left.1.6 \mathrm{~Hz}, H 4^{\prime \prime \prime}\right), 8.17(1 \mathrm{H}, \mathrm{tt}, J=7.8$ and $1.3 \mathrm{~Hz}, H 4)$, $8.13\left(1 \mathrm{H}, \mathrm{d}, J=1.6 \mathrm{~Hz}, H 6^{\prime}\right), 7.97\left(1 \mathrm{H}, \mathrm{d}, J=1.6 \mathrm{~Hz}, H 3^{\prime}\right)$, $7.91(2 \mathrm{H}, \mathrm{dd}, J=7.8$ and $7.0 \mathrm{~Hz}, H 3(5)), 7.46(1 \mathrm{H}, \mathrm{ddd}, J=8.1$, 4.8 and $\left.0.7 \mathrm{~Hz}, H 5^{\prime \prime \prime}\right) ;{ }^{13} \mathrm{C}$ NMR (75 MHz, $\left.\mathrm{CD}_{3} \mathrm{OD}\right): \delta 161.0$ $\left(C 2^{\prime}\right), 147.7\left(C 6^{\prime \prime \prime}\right), 146.1\left(C 2^{\prime \prime \prime}\right), 144.7(C 2(6)), 139.2(C 4)$, $138.9\left(C 6^{\prime}\right), 137.0\left(C 3^{\prime}\right), 135.3\left(C 3^{\prime \prime \prime}\right), 135.2\left(C 5^{\prime}\right), 133.6$ $\left(C 4^{\prime \prime \prime}\right), 128.5(C 3(5)), 125.1\left(C 5^{\prime \prime \prime}\right)$. MS (CI, $\left.\mathrm{m} / \mathrm{z}\right): 250$ (100, $\mathrm{M}+1$ ). Anal. Calcd for $\mathrm{C}_{14} \mathrm{H}_{11} \mathrm{~N}_{5} \cdot 1.4 \mathrm{H}_{2} \mathrm{O}: \mathrm{C}, 66.26 ; \mathrm{H}, 4.57$; N, 27.60. Found: C, 66.58; H, 4.43; N, 27.31.

\subsubsection{8. $N$-(5-Phenylpyrimidin-2-yl)pyridinium aminide (5a)}

Yellow solid $(186 \mathrm{mg}, 75 \%)$, mp hydrobromide 222$223{ }^{\circ} \mathrm{C}$ (yellowish solid, ethanol/ethyl acetate); IR (KBr) $\nu_{\max }\left(\mathrm{cm}^{-1}\right)$ : 1600,$1438 ;{ }^{1} \mathrm{H}$ NMR $\left(300 \mathrm{MHz}, \mathrm{CD}_{3} \mathrm{OD}\right)$ : $\delta 8.75(2 \mathrm{H}, \mathrm{dd}, J=6.9$ and $1.3 \mathrm{~Hz}, H 2(6)), 8.40(2 \mathrm{H}, \mathrm{s}$, 
$\left.H 4^{\prime}\left(6^{\prime}\right)\right), 8.19(1 \mathrm{H}, \mathrm{tt}, J=7.8$ and $1.3 \mathrm{~Hz}, H 4), 7.91(2 \mathrm{H}, \mathrm{dd}$, $J=7.8$ and $6.9 \mathrm{~Hz}, H 3(5)), 7.49(2 \mathrm{H}, \mathrm{dd}, J=8.4$ and $1.4 \mathrm{~Hz}$, $\left.H 2^{\prime \prime \prime}\left(6^{\prime \prime \prime}\right)\right), 7.41\left(2 \mathrm{H}\right.$, dd, $J=8.4$ and $\left.7.2 \mathrm{~Hz}, H 3^{\prime \prime \prime}\left(5^{\prime \prime \prime}\right)\right), 7.29$ $\left(1 \mathrm{H}, \mathrm{tt}, J=7.2\right.$ and $\left.1.4 \mathrm{~Hz}, H 4^{\prime \prime \prime}\right) ;{ }^{13} \mathrm{C}$ NMR $(75 \mathrm{MHz}$, $\left.\mathrm{CD}_{3} \mathrm{OD}\right): \delta 168.8\left(C 2^{\prime}\right), 157.2\left(C 4^{\prime}\left(6^{\prime}\right)\right), 145.7(C 2(6))$, $139.8(C 4), 137.0\left(C 1^{\prime \prime \prime}\right), 130.1\left(C 3^{\prime \prime \prime}\left(5^{\prime \prime \prime}\right)\right), 128.7(C 3(5))$, $127.8\left(C 4^{\prime \prime \prime}\right), 126.1\left(C 2^{\prime \prime \prime}\left(6^{\prime \prime \prime}\right)\right), 122.4\left(C 5^{\prime}\right)$. MS (CI, $\left.m / z\right): 249$ $(100, \quad M+1), \quad 200$ (56), 172 (24). Anal. Calcd for $\mathrm{C}_{15} \mathrm{H}_{12} \mathrm{~N}_{4} \cdot \mathrm{HBr} \cdot \mathrm{H}_{2} \mathrm{O}: \mathrm{C}, 52.02 ; \mathrm{H}, 4.37 ; \mathrm{N}, 16.19$. Found: $\mathrm{C}$, 51.96; H, 3.98; N, 16.03 .

\subsubsection{N-[5-(4-Methylphenyl)pyrimidin-2-yl]pyridinium aminide $(\mathbf{5 b})$}

Yellow solid (184 mg, 70\%), mp hydrobromide $242-244{ }^{\circ} \mathrm{C}$ (yellowish solid, ethanol/ethyl acetate); IR $(\mathrm{KBr}) \nu_{\max }\left(\mathrm{cm}^{-1}\right)$ : 1600,$1437 ; \nu_{\max }(\mathrm{KBr} /$ hydrobromide) $2813,1619,1594$, 1468, 1431, 818, 773, 669; ${ }^{1} \mathrm{H}$ NMR (300 MHz, $\left.\mathrm{CD}_{3} \mathrm{OD}\right)$ : $\delta 8.74(2 \mathrm{H}, \mathrm{dd}, J=6.9$ and $1.3 \mathrm{~Hz}, H 2(6)), 8.36(2 \mathrm{H}, \mathrm{s}$, $\left.H 4^{\prime}\left(6^{\prime}\right)\right), 8.17(1 \mathrm{H}, \mathrm{tt}, J=7.8$ and $1.3 \mathrm{~Hz}, H 4), 7.89(2 \mathrm{H}, \mathrm{dd}, J=$ 7.8 and $6.9 \mathrm{~Hz}, H 3(5)), 7.36\left(2 \mathrm{H}, \mathrm{d}, J=8.2 \mathrm{~Hz}, H 2^{\prime \prime \prime}\left(6^{\prime \prime \prime}\right)\right), 7.22$ $\left(2 \mathrm{H}, \mathrm{d}, J=8.2 \mathrm{~Hz}, \mathrm{H3}^{\prime \prime \prime}\left(5^{\prime \prime \prime}\right)\right), 2.36\left(3 \mathrm{H}, \mathrm{s}, \mathrm{CH}_{3}\right) ;{ }^{13} \mathrm{C}$ NMR $\left(75 \mathrm{MHz}, \mathrm{CD}_{3} \mathrm{OD}\right): \delta 168.6\left(C 2^{\prime}\right), 157.0\left(C 4^{\prime}\left(6^{\prime}\right)\right), 145.6$ $(C 2(6)), 139.6(C 4), 137.7\left(C 4^{\prime \prime \prime}\right), 134.1 \quad\left(C 1^{\prime \prime \prime}\right), 130.7$ $\left(C 3^{\prime \prime \prime}\left(5^{\prime \prime \prime}\right)\right), 128.7(C 3(5)), 126.0\left(C 2^{\prime \prime \prime}\left(6^{\prime \prime \prime}\right)\right), 122.5\left(C 5^{\prime}\right), 21.1$ $\left(\mathrm{CH}_{3}\right)$. MS (CI, m/z): $263(35, \mathrm{M}+1), 186(89), 80$ (100). Anal. Calcd for $\mathrm{C}_{16} \mathrm{H}_{14} \mathrm{~N}_{4} \cdot \mathrm{HBr} \cdot 1 / 2 \mathrm{H}_{2} \mathrm{O}: \mathrm{C}, 54.69 ; \mathrm{H}, 4.31 ; \mathrm{N}$, 15.96. Found: C, 54.66; H, 4.41; N, 15.74 .

\subsubsection{N-[5-(4-Methoxyphenyl)pyrimidin-2-yl]pyridinium aminide $(5 \boldsymbol{c})$}

Yellow solid (225 mg, 81\%), mp hydrobromide 235$236{ }^{\circ} \mathrm{C}$ (yellowish solid, ethanol); IR $(\mathrm{KBr}) \nu_{\max }\left(\mathrm{cm}^{-1}\right)$ : $1592,1420,1246,820,776 ;{ }^{1} \mathrm{H}$ NMR (300 MHz, $\left.\mathrm{CD}_{3} \mathrm{OD}\right)$ : $\delta 8.74(2 \mathrm{H}, \mathrm{dd}, J=7.0$ and $1.3 \mathrm{~Hz}, H 2(6)), 8.33(1 \mathrm{H}, \mathrm{s}$, $\left.H 4^{\prime}\left(6^{\prime}\right)\right), 8.16(1 \mathrm{H}, \mathrm{tt}, J=7.7$ and $1.3 \mathrm{~Hz}, H 4), 7.89(2 \mathrm{H}$, dd, $J=7.7$ and $7.0 \mathrm{~Hz}, H 3(5)), 7.39\left(2 \mathrm{H}, \mathrm{d}, J=8.9 \mathrm{~Hz}, H 2^{\prime \prime \prime}\left(6^{\prime \prime \prime}\right)\right)$, $6.97\left(2 \mathrm{H}, \mathrm{d}, J=8.9 \mathrm{~Hz}, H 3^{\prime \prime \prime}\left(5^{\prime \prime \prime}\right)\right), 3.82\left(3 \mathrm{H}, \mathrm{s}, \mathrm{CH}_{3}\right) ;{ }^{13} \mathrm{C}$ NMR (75 MHz, $\left.\mathrm{CD}_{3} \mathrm{OD}\right): \delta 168.2\left(C 2^{\prime}\right), 160.0\left(C 4^{\prime \prime \prime}\right), 156.6$ $\left(C 4^{\prime}\left(6^{\prime}\right)\right), 145.4(C 2(6)), 139.4(C 4), 129.3\left(C 1^{\prime \prime \prime}\right), 128.5$ $(C 3(5)), 127.2\left(C 2^{\prime \prime \prime}\left(6^{\prime \prime \prime}\right)\right), 122.3\left(C 5^{\prime}\right), 115.4\left(C 3^{\prime \prime \prime}\left(5^{\prime \prime \prime}\right)\right), 55.7$ $\left(\mathrm{CH}_{3}\right)$. MS (CI, m/z): 279 (100, M+1), 278 (32), 253 (33), 251 (34), 202 (57), 172 (20), 80 (34). Anal. Calcd for $\mathrm{C}_{16} \mathrm{H}_{14} \mathrm{~N}_{4} \mathrm{O} \cdot \mathrm{HBr} \cdot 1 / 4 \mathrm{H}_{2} \mathrm{O}: \mathrm{C}, 52.83 ; \mathrm{H}, 4.30 ; \mathrm{N}, 15.40$. Found: C, 52.65; H, 4.08; N, 15.21.

\subsubsection{1. $\mathrm{N}$-[5-(trans-2-Phenylvinyl)pyrimidin-2-yl]- pyridinium aminide $(\mathbf{5} \boldsymbol{d})$}

Yellow solid (200 mg, 73\%), mp hydrobromide $227-229^{\circ} \mathrm{C}$ (yellowish solid, ethanol/ethyl acetate); IR $(\mathrm{KBr}) \nu_{\max }\left(\mathrm{cm}^{-1}\right)$ : 1591,$1438 ;{ }^{1} \mathrm{H}$ NMR $\left(300 \mathrm{MHz}, \mathrm{CD}_{3} \mathrm{OD}\right): \delta 8.71(2 \mathrm{H}, \mathrm{dd}, J=$ 6.8 and $1.2 \mathrm{~Hz}, H 2(6)), 8.32\left(2 \mathrm{H}, \mathrm{s}, H 4^{\prime}\left(6^{\prime}\right)\right), 8.16(1 \mathrm{H}, \mathrm{tt}, J=7.8$ and $1.2 \mathrm{~Hz}, H 4), 7.87(2 \mathrm{H}, \mathrm{dd}, J=7.8$ and $6.8 \mathrm{~Hz}, H 3(5)), 7.47$ $\left(2 \mathrm{H}, \mathrm{m}, H 2^{\prime \prime \prime}\left(6^{\prime \prime \prime}\right)\right), 7.31\left(2 \mathrm{H}\right.$, ap t, $\left.J=7.4 \mathrm{~Hz}, H 3^{\prime \prime \prime}\left(5^{\prime \prime \prime}\right)\right), 7.20$ $\left(1 \mathrm{H}, \mathrm{tt}, J=7.4\right.$ and $\left.1.3 \mathrm{~Hz}, H 4^{\prime \prime \prime}\right), 6.92\left(2 \mathrm{H}, \mathrm{m}, H_{\alpha}\right.$ and $\left.H_{\beta}\right)$; ${ }^{13} \mathrm{C}$ NMR (75 MHz, $\left.\mathrm{CD}_{3} \mathrm{OD}\right): \delta 168.1\left(C 2^{\prime}\right), 156.8\left(C 4^{\prime}\left(6^{\prime}\right)\right)$, 145.1 (C2(6)), $139.4(C 4), 138.6\left(C 1^{\prime \prime \prime}\right), 129.3\left(C 3^{\prime \prime \prime}\left(5^{\prime \prime \prime}\right)\right)$, $128.3(C 3(5)), 127.8\left(C 4^{\prime \prime \prime}\right), 126.8\left(C 2^{\prime \prime \prime}\left(6^{\prime \prime \prime}\right)\right), 125.5\left(C_{\beta}\right)$,
$123.3\left(C_{\alpha}\right), 119.4\left(C 5^{\prime}\right)$. MS (CI, m/z): $275(100, \mathrm{M}+1)$, 274 (26), 198 (84), 80 (79). Anal. Calcd for $\mathrm{C}_{17} \mathrm{H}_{14} \mathrm{~N}_{4} \cdot \mathrm{HBr}$. $1 / 4 \mathrm{H}_{2} \mathrm{O}: \mathrm{C}, 56.59 ; \mathrm{H}, 4.63 ; \mathrm{N}, 15.53$. Found: $\mathrm{C}, 56.93 ; \mathrm{H}$, $4.36 ; \mathrm{N}, 15.43$.

\subsubsection{2. $N$-[5-(Pyridin-3-yl)pyrimidin-2-yl]pyridinium aminide $(5 \boldsymbol{e})$}

Yellow oil $(236 \mathrm{mg}, 95 \%), \mathrm{mp}$ hydrobromide $>300{ }^{\circ} \mathrm{C}$ (yellowish solid, ethanol); IR $(\mathrm{KBr}) \nu_{\max }\left(\mathrm{cm}^{-1}\right)$ : 1602, 1446, 1307, 668; ${ }^{1} \mathrm{H}$ NMR (300 MHz, CD $\left.\mathrm{CD}_{3} \mathrm{OD}\right): \delta 8.77(2 \mathrm{H}$, dd, $J=6.9$ and $1.3 \mathrm{~Hz}, H 2(6)), 8.69(1 \mathrm{H}, \mathrm{dd}, J=2.4$ and $\left.0.9 \mathrm{~Hz}, H 2^{\prime \prime \prime}\right), 8.45\left(1 \mathrm{H}, \mathrm{dd}, J=4.8\right.$ and $\left.1.6 \mathrm{~Hz}, H 6^{\prime \prime \prime}\right), 8.44$ $\left(1 \mathrm{H}, \mathrm{s}, H 4^{\prime}\left(6^{\prime}\right)\right), 8.23(1 \mathrm{H}, \mathrm{tt}, J=7.7$ and $1.3 \mathrm{~Hz}, H 4), 7.98$ $\left(1 \mathrm{H}\right.$, ddd, $J=8.1,2.4$ and $\left.1.6 \mathrm{~Hz}, H 4^{\prime \prime \prime}\right), 7.94(2 \mathrm{H}, \mathrm{dd}, J=7.7$ and $6.9 \mathrm{~Hz}, H 3(5)), 7.47(1 \mathrm{H}$, ddd, $J=8.1,4.8$ and $0.9 \mathrm{~Hz}$, $\left.H 5^{\prime \prime \prime}\right) ;{ }^{13} \mathrm{C}$ NMR (75 MHz, $\left.\mathrm{CD}_{3} \mathrm{OD}\right): \delta 168.6\left(C 2^{\prime}\right), 156.9$ $\left(C 4^{\prime}\left(6^{\prime}\right)\right), 147.6\left(C 6^{\prime \prime \prime}\right), 146.1\left(C 2^{\prime \prime \prime}\right), 145.3(C 2(6)), 139.8(C 4)$, $134.1\left(C 4^{\prime \prime \prime}\right), 133.5\left(C 3^{\prime \prime \prime}\right), 128.4(C 3(5)), 125.2\left(C 5^{\prime \prime \prime}\right), 118.1$ $\left(C 5^{\prime}\right)$. MS (CI, m/z): $250(100, \mathrm{M}+1), 249$ (19), 201 (70), 173 (34), 80 (34). Anal. Calcd for $\mathrm{C}_{14} \mathrm{H}_{11} \mathrm{~N}_{5} \cdot 2 \mathrm{HBr} \cdot \mathrm{H}_{2} \mathrm{O}$ : C, 39.19; H, 3.52; N, 16.32. Found: C, 39.74; H, 3.41; N, 15.99.

\subsection{Synthesis of symmetrical 3,5-disubstituted aminides 6 and 7: general procedure}

Method A. Dibromoaminides $\mathbf{2 d}, \mathbf{e}^{8 \mathrm{c}}(1 \mathrm{mmol})$ and the corresponding boronic acid ( $3 \mathrm{mmol}$ ) were dissolved in a toluene/ethanol mixture $(20: 1,15 \mathrm{~mL}) . \mathrm{Cs}_{2} \mathrm{CO}_{3}(4 \mathrm{mmol})$ was added, followed by $\mathrm{Pd}\left(\mathrm{PPh}_{3}\right)_{4}(5 \mathrm{~mol} \%)$. After the addition, the mixture was kept under argon with vigorous stirring for $5 \mathrm{~min}$, and then heated under reflux for the reaction time indicated in Table 2.

Method B. Dibromoaminides $2 \mathbf{d}, \mathbf{e}^{8 \mathrm{c}}(1 \mathrm{mmol})$ and the corresponding boronic acid ( $3 \mathrm{mmol}$ ) were dissolved in a toluene/ethanol mixture $(4: 1,10 \mathrm{~mL}) . \mathrm{K}_{2} \mathrm{CO}_{3}(20 \mathrm{mmol})$ was added followed by $\mathrm{Pd}\left(\mathrm{PPh}_{3}\right)_{4}(5 \mathrm{~mol} \%)$. After the addition, the mixture was kept under argon with vigorous stirring for $5 \mathrm{~min}$, and then heated under reflux for the reaction time indicated in Table 2.

In both methods, as soon as the starting material has been consumed, the system was allowed to reach room temperature, the mixture was filtered through Celite or silica gel and the residue washed with acetonitrile until colour was no longer observed in the filtrate. The filtrates were combined, the solvent evaporated to dryness and the residue purified by flash chromatography through a silica gel column, using ethanol as the mobile phase. Finally, the compounds were, if possible, recrystallized from a suitable solvent. Compounds $\mathbf{6 b}$ and $\mathbf{6 h}$ were isolated as yellow oils. Compounds $6 \mathbf{6}, \mathbf{6 e}, \mathbf{6 f}, \mathbf{6 g}, \mathbf{7 b}$, $\mathbf{7 d}, 7 \mathbf{e}$ and $7 \mathbf{f}$ were transformed into the corresponding hydrobromides by adding aqueous hydrogen bromide (48\%, 3 equiv) and the salts were isolated after removal of excess acid. Ethyl acetate was then added and the aminide hydrobromide was treated in an ultrasonic bath. The remaining solid was filtered off and dried. Hydrobromides of compounds 6a, $\mathbf{6 g}, 7 \mathbf{b}$ and $7 \mathbf{d}$ were purified by recrystallization from ethanol. To recover the free aminides $6 \mathbf{e}, \mathbf{6 f}, \mathbf{7 e}$ and $\mathbf{7 f}$, the 
corresponding hydrobromide was poured into acetone and ion exchange resin Amberlite IRA-67 ( 3 equiv $C_{\mathrm{E}}=1.6$ equiv/g) was added. When equilibration was complete, the resin was removed by filtration and the solvent evaporated to give the desired aminide, which was used without further purification.

\subsubsection{N-(3,5-Diphenylpyridin-2-yl)pyridinium aminide (6a)}

See Ref. 10a.

\subsubsection{N-[3,5-Bis(4-methylphenyl)pyridin-2-yl]pyridinium aminide $(\boldsymbol{6} \boldsymbol{b})$}

Yellow oil $(197 \mathrm{mg}, 56 \%)$; IR $(\mathrm{KBr}) \nu_{\max }\left(\mathrm{cm}^{-1}\right): 1595$, 1435, 1380, 1308, 1165, 815; ${ }^{1} \mathrm{H}$ NMR (300 MHz, $\left.\mathrm{CD}_{3} \mathrm{OD}\right)$ : $\delta 8.62(2 \mathrm{H}, \mathrm{dd}, J=7.0$ and $1.2 \mathrm{~Hz}, H 2(6)), 7.92(1 \mathrm{H}, \mathrm{tt}, J=7.8$ and $1.2 \mathrm{~Hz}, H 4), 7.90\left(1 \mathrm{H}, \mathrm{d}, J=2.5 \mathrm{~Hz}, H 6^{\prime}\right), 7.70(2 \mathrm{H}, \mathrm{dd}$, $J=7.8$ and $7.0 \mathrm{~Hz}, H 3(5)), 7.60\left(2 \mathrm{H}, \mathrm{d}, J=8.1 \mathrm{~Hz}, H 2^{\prime \prime}\left(6^{\prime \prime}\right)\right)$, $7.54\left(1 \mathrm{H}, \mathrm{d}, J=2.5 \mathrm{~Hz}, H 4^{\prime}\right), 7.39(2 \mathrm{H}, \mathrm{d}, J=8.2 \mathrm{~Hz}$, $\left.H 2^{\prime \prime \prime}\left(6^{\prime \prime \prime}\right)\right), 7.22\left(2 \mathrm{H}\right.$, br d, $\left.J=7.8 \mathrm{~Hz}, H 3^{\prime \prime}\left(5^{\prime \prime}\right)\right), 7.18(2 \mathrm{H}, \mathrm{br} \mathrm{d}$, $\left.J=7.9 \mathrm{~Hz}, \mathrm{H}^{\prime \prime \prime}\left(5^{\prime \prime \prime}\right)\right), 2.37\left(3 \mathrm{H}, \mathrm{s}, \mathrm{CH}_{3}\right), 2.33\left(3 \mathrm{H}, \mathrm{s}, \mathrm{CH}_{3}\right)$; ${ }^{13} \mathrm{C}$ NMR (125 MHz, CD $\left.{ }_{3} \mathrm{OD}\right): \delta 162.9\left(C 2^{\prime}\right), 144.8(C 2(6))$, $143.6\left(C 6^{\prime}\right), 138.1\left(C 1^{\prime \prime}\right), 137.6\left(C 4^{\prime \prime}\right), 137.3\left(C 4^{\prime}\right), 137.1\left(C 1^{\prime \prime \prime}\right)$, $136.9(C 4), 136.9\left(C 4^{\prime \prime \prime}\right), 130.5\left(C 3^{\prime \prime \prime}\left(5^{\prime \prime \prime}\right)\right), 130.4\left(C 2^{\prime \prime}\left(6^{\prime \prime}\right)\right)$, $129.8\left(C 3^{\prime \prime}\left(5^{\prime \prime}\right)\right), 128.2(C 3(5)), 126.3\left(C 2^{\prime \prime \prime}\left(6^{\prime \prime \prime}\right)\right), 125.6\left(C 5^{\prime}\right)$, $124.9\left(\mathrm{C3}^{\prime}\right), 21.3\left(\mathrm{CH}_{3}\right), 21.1\left(\mathrm{CH}_{3}\right)$. HRMS (FAB) calcd for $\mathrm{C}_{24} \mathrm{H}_{22} \mathrm{~N}_{3}:[\mathrm{M}+\mathrm{H}]^{+}$352.1814, found: 352.1829 .

\subsubsection{N-[3,5-Bis(4-hydroxymethylphenyl)pyridin-2-yl]- pyridinium aminide $(\boldsymbol{6} \boldsymbol{c})$}

Red solid (329 mg, 86\%, ethanol/toluene); mp 205-206 ${ }^{\circ} \mathrm{C}$; IR $(\mathrm{KBr}) \nu_{\max }\left(\mathrm{cm}^{-1}\right): 3169,3112,1599,1442,1419,1384$, 1321， 1041，824， 678，594， 517; ${ }^{1} \mathrm{H}$ NMR $(500 \mathrm{MHz}$, $\left.\mathrm{CD}_{3} \mathrm{OD}\right): \delta 8.72(2 \mathrm{H}, \mathrm{dd}, J=7.0$ and $1.2 \mathrm{~Hz}, H 2(6)), 8.06(1 \mathrm{H}$, tt, $J=7.8$ and $1.2 \mathrm{~Hz}, H 4), 7.96\left(1 \mathrm{H}, \mathrm{d}, J=2.4 \mathrm{~Hz}, H 6^{\prime}\right), 7.82$ $(2 \mathrm{H}, \mathrm{dd}, J=7.8$ and $7.0 \mathrm{~Hz}, H 3(5)), 7.73(2 \mathrm{H}, \mathrm{d}, J=8.3 \mathrm{~Hz}$, $\left.H 2^{\prime \prime}\left(6^{\prime \prime}\right)\right), 7.62\left(1 \mathrm{H}, \mathrm{d}, J=2.4 \mathrm{~Hz}, H 4^{\prime}\right), 7.53(2 \mathrm{H}, \mathrm{d}, J=8.5 \mathrm{~Hz}$, $\left.H 2^{\prime \prime \prime \prime}\left(6^{\prime \prime \prime \prime}\right)\right), 7.44\left(2 \mathrm{H}, \mathrm{d}, J=8.4 \mathrm{~Hz}, H 3^{\prime \prime}\left(5^{\prime \prime}\right)\right), 7.40(2 \mathrm{H}, \mathrm{d}, J=$ $\left.8.6 \mathrm{~Hz}, \mathrm{H3}^{\prime \prime \prime}\left(5^{\prime \prime \prime}\right)\right), 4.68\left(2 \mathrm{H}, \mathrm{s}, \mathrm{CH}_{2} \mathrm{O}\right), 4.64\left(2 \mathrm{H}, \mathrm{s}, \mathrm{CH}_{2} \mathrm{O}\right)$; ${ }^{13} \mathrm{C}$ NMR (125 MHz, CD $\left.3 \mathrm{OD}\right): \delta 161.3\left(C 2^{\prime}\right), 145.4(C 2(6))$, $144.1\left(C 6^{\prime}\right), 141.5\left(C 4^{\prime \prime}\right), 140.8\left(C 4^{\prime \prime \prime}\right), 139.8\left(C 1^{\prime \prime}\right), 139.0$ $\left(C 1^{\prime \prime \prime}\right), 138.2(C 4), 137.5\left(C 4^{\prime}\right), 130.4\left(C 2^{\prime \prime}\left(6^{\prime \prime}\right)\right), 128.7$ $\left(C 3^{\prime \prime \prime}\left(5^{\prime \prime \prime}\right)\right), 128.5(C 3(5)), 127.9\left(C 3^{\prime \prime}\left(5^{\prime \prime}\right)\right), 126.4\left(C 2^{\prime \prime \prime}\left(6^{\prime \prime \prime}\right)\right)$, $125.8\left(C 5^{\prime}\right), 124.8\left(C 3^{\prime}\right), 65.1\left(C_{2} \mathrm{O}\right), 65.0\left(C \mathrm{H}_{2} \mathrm{O}\right) . \mathrm{MS}(\mathrm{CI}$, $\mathrm{m} / \mathrm{z}): 384$ (4, M+1), 287 (27), 276 (25), 275 (100). Anal. Calcd for $\mathrm{C}_{24} \mathrm{H}_{21} \mathrm{~N}_{3} \mathrm{O}_{2} \cdot 1 / 2 \mathrm{H}_{2} \mathrm{O}$ : C, 73.45; H, 5.65; N, 10.71. Found: C, 73.34; H, 5.47; N, 10.60 .

\subsubsection{N-[3,5-Bis(4-methoxyphenyl)pyridin-2-yl]pyridinium aminide $(\boldsymbol{6} \boldsymbol{d})$}

Red solid (345 mg, 90\%, ethanol), mp 94-96 ${ }^{\circ} \mathrm{C}$; IR ( $\left.\mathrm{KBr}\right)$ $\nu_{\max }\left(\mathrm{cm}^{-1}\right): 1594,1510,1436,1418,1403,1241 ;{ }^{1} \mathrm{H} \mathrm{NMR}$ $\left(300 \mathrm{MHz}, \mathrm{CD}_{3} \mathrm{OD}\right): \delta 8.68(2 \mathrm{H}, \mathrm{dd}, J=7.0$ and $1.3 \mathrm{~Hz}$, $H 2(6)), 7.98(1 \mathrm{H}, \mathrm{tt}, J=7.6$ and $1.3 \mathrm{~Hz}, H 4), 7.85(1 \mathrm{H}, \mathrm{d}, J=$ $\left.2.3 \mathrm{~Hz}, H 6^{\prime}\right), 7.70(2 \mathrm{H}, \mathrm{dd}, J=7.6$ and $7.0 \mathrm{~Hz}, H 3(5)), 7.66$ $\left(2 \mathrm{H}, \mathrm{d}, J=8.8 \mathrm{~Hz}, H 2^{\prime \prime}\left(6^{\prime \prime}\right)\right), 7.53\left(1 \mathrm{H}, \mathrm{d}, J=2.3 \mathrm{~Hz}, H 4^{\prime}\right)$, $7.44\left(2 \mathrm{H}, \mathrm{d}, J=8.8 \mathrm{~Hz}, H 2^{\prime \prime \prime}\left(6^{\prime \prime \prime}\right)\right), 6.98(2 \mathrm{H}, \mathrm{d}, J=8.8 \mathrm{~Hz}$, $\left.H 3^{\prime \prime}\left(5^{\prime \prime}\right)\right), 6.96\left(2 \mathrm{H}, \mathrm{d}, J=8.8 \mathrm{~Hz}, H 3^{\prime \prime \prime}\left(5^{\prime \prime \prime}\right)\right), 3.85(3 \mathrm{H}, \mathrm{s}$, $\left.\mathrm{OCH}_{3}\right), 3.83\left(3 \mathrm{H}, \mathrm{s}, \mathrm{OCH}_{3}\right) ;{ }^{13} \mathrm{C} \mathrm{NMR}\left(75 \mathrm{MHz}, \mathrm{CD}_{3} \mathrm{OD}\right)$ : $\delta 162.3\left(C 2^{\prime}\right), 159.8\left(C 4^{\prime \prime \prime}\right), 159.6\left(C 4^{\prime \prime}\right), 144.5(C 2(6))$, $142.6\left(C 6^{\prime}\right), 136.8\left(C 4^{\prime}\right), 136.7(C 4), 133.0\left(C 1^{\prime \prime}\right), 132.3$ $\left(C 1^{\prime \prime \prime}\right), 131.2\left(C 2^{\prime \prime}\left(6^{\prime \prime}\right)\right), 128.0(C 3(5)), 127.2\left(C 2^{\prime \prime \prime}\left(6^{\prime \prime \prime}\right)\right)$, $125.4\left(C 5^{\prime}\right), 124.5\left(C 3^{\prime}\right), 115.1\left(C 3^{\prime \prime \prime}\left(5^{\prime \prime \prime}\right)\right), 114.3\left(C 3^{\prime \prime}\left(5^{\prime \prime}\right)\right)$, $55.7\left(\mathrm{OCH}_{3}\right)$. MS (CI, m/z): 384 (100, M+1), 383 (65), 307 (54), 305 (46), 108 (17), 80 (63). Anal. Calcd for $\mathrm{C}_{24} \mathrm{H}_{21} \mathrm{~N}_{3} \mathrm{O}_{2} \cdot \mathrm{H}_{2} \mathrm{O}: \mathrm{C}, 71.80 ; \mathrm{H}, 5.77 ; \mathrm{N}, 10.47$. Found: $\mathrm{C}$, 72.13; H, 5.51; N, 10.31 .

\subsubsection{N-[3,5-Bis(3,5-dimethylphenyl)pyridin-2-yl]- pyridinium aminide $(\boldsymbol{6} \boldsymbol{e})$}

Red solid (265 mg, 70\%); mp 77-79 ${ }^{\circ} \mathrm{C}$; IR (KBr) $\nu_{\max }$ $\left(\mathrm{cm}^{-1}\right)$ : 2913, 1591, 1402, 1374, 1320, 1143, 1008, 845, 757, 698, 669; ${ }^{1} \mathrm{H}$ NMR (500 MHz, CD $\left.3 \mathrm{OD}\right): \delta 8.69(2 \mathrm{H}$, $\mathrm{dd}, J=7.0$ and $1.2 \mathrm{~Hz}, H 2(6)), 8.04(1 \mathrm{H}, \mathrm{tt}, J=7.7$ and $1.2 \mathrm{~Hz}, H 4), 7.92\left(1 \mathrm{H}, \mathrm{d}, J=2.4 \mathrm{~Hz}, H 6^{\prime}\right), 7.81(2 \mathrm{H}, \mathrm{dd}$, $J=7.7$ and $7.0 \mathrm{~Hz}, H 3(5)), 7.55\left(1 \mathrm{H}, \mathrm{d}, J=2.4 \mathrm{~Hz}, H 4^{\prime}\right), 7.32$ $\left(2 \mathrm{H}, \mathrm{d}, J=1.6 \mathrm{~Hz}, H 2^{\prime \prime}\left(6^{\prime \prime}\right)\right), 7.14\left(2 \mathrm{H}, \mathrm{d}, J=1.6 \mathrm{~Hz}, H 2^{\prime \prime \prime}\left(6^{\prime \prime \prime}\right)\right)$, $6.99\left(1 \mathrm{H}, \mathrm{t}, J=1.6 \mathrm{~Hz}, H 4^{\prime \prime}\right), 6.92\left(1 \mathrm{H}, \mathrm{t}, J=1.6 \mathrm{~Hz}, H 4^{\prime \prime \prime}\right), 2.38$ $\left(6 \mathrm{H}, \mathrm{s}, \mathrm{CH}_{3}\right), 2.35\left(6 \mathrm{H}, \mathrm{s}, \mathrm{CH}_{3}\right) ;{ }^{13} \mathrm{C} \mathrm{NMR}(125 \mathrm{MHz}$, $\left.\mathrm{CD}_{3} \mathrm{OD}\right): \delta 162.8\left(C 2^{\prime}\right), 145.4(C 2(6)), 144.0\left(C 6^{\prime}\right), 140.9$ $\left(C 1^{\prime \prime}\right), 140.0\left(C 1^{\prime \prime \prime}\right), 139.6(C 4), 138.9\left(C 4^{\prime}\right), 138.0\left(C 3^{\prime \prime \prime}\left(5^{\prime \prime \prime}\right)\right)$, $137.8\left(C 3^{\prime \prime}\left(5^{\prime \prime}\right)\right), 129.7\left(C 4^{\prime \prime}\right), 129.0\left(C 4^{\prime \prime \prime}\right), 128.6(C 3(5))$, $128.4\left(C 2^{\prime \prime}\left(6^{\prime \prime}\right)\right), 126.4\left(C 5^{\prime}\right), 125.5\left(C 3^{\prime}\right), 124.5\left(C 2^{\prime \prime \prime}\left(6^{\prime \prime \prime}\right)\right)$, $21.7\left(\mathrm{CH}_{3}\right), 21.6\left(\mathrm{CH}_{3}\right)$. MS(CI, $\left.\mathrm{m} / \mathrm{z}\right): 380(7, \mathrm{M}+1), 303$ (40), 301 (100), 146 (23). Anal. Calcd for $\mathrm{C}_{26} \mathrm{H}_{25} \mathrm{~N}_{3} \cdot 1 / 2 \mathrm{H}_{2} \mathrm{O}$ : C, 80.38; H, 6.75; N, 10.82. Found: C, 80.47; H, 6.51; N, 10.52.

\subsection{6. $N$-[3,5-Bis(3-chlorophenyl)pyridin-2-yl]pyridinium aminide $(\boldsymbol{6} \boldsymbol{f})$}

Red solid (305 mg, 78\%); mp $71-73{ }^{\circ} \mathrm{C}$; IR (KBr) $\nu_{\max }$ $\left(\mathrm{cm}^{-1}\right)$ : 1590, 1473, 1398, 1314, 1145, 1017, 785, 762, 695, 668; ${ }^{1} \mathrm{H}$ NMR (500 MHz, $\left.\mathrm{CD}_{3} \mathrm{OD}\right): \delta 8.68(2 \mathrm{H}, \mathrm{dd}, J=7.0$ and $1.3 \mathrm{~Hz}, H 2(6)), 8.05(1 \mathrm{H}, \mathrm{tt}, J=7.7$ and $1.3 \mathrm{~Hz}, H 4), 7.93$ $\left(1 \mathrm{H}, \mathrm{d}, J=2.6 \mathrm{~Hz}, H 6^{\prime}\right), 7.81\left(3 \mathrm{H}, \mathrm{m}, H 3(5)\right.$ and $\left.H 2^{\prime \prime}\right), 7.62$ $\left(1 \mathrm{H}\right.$, ap dt, $J=7.7$ and $\left.1.6 \mathrm{~Hz}, H 6^{\prime \prime}\right), 7.55(1 \mathrm{H}, \mathrm{d}, J=2.6 \mathrm{~Hz}$, $\left.H 4^{\prime}\right), 7.52\left(1 \mathrm{H}\right.$, ap t, $\left.J=1.9 \mathrm{~Hz}, H 2^{\prime \prime \prime}\right), 7.43(1 \mathrm{H}$, ddd, $J=7.7$, 1.6 and $\left.1.0 \mathrm{~Hz}, H 6^{\prime \prime \prime}\right), 7.40\left(1 \mathrm{H}\right.$, ap t, $\left.J=7.7 \mathrm{~Hz}, H 5^{\prime \prime}\right), 7.34$ $\left(1 \mathrm{H}\right.$, ap t, $\left.J=7.7 \mathrm{~Hz}, H 5^{\prime \prime \prime}\right), 7.31(1 \mathrm{H}$, ddd, $J=7.9,1.9$ and $\left.1.0 \mathrm{~Hz}, H 4^{\prime \prime}\right), 7.23\left(1 \mathrm{H}\right.$, ddd, $J=7.9,1.9$ and $\left.1.0 \mathrm{~Hz}, H 4^{\prime \prime \prime}\right)$; ${ }^{13} \mathrm{C}$ NMR (75 MHz, $\left.\left(\mathrm{CD}_{3}\right)_{2} \mathrm{CO}\right): \delta 161.8\left(C 2^{\prime}\right), 145.3(C 2(6))$, $143.2\left(C 6^{\prime}\right), 143.2\left(C 1^{\prime \prime}\right), 142.2\left(C 1^{\prime \prime \prime}\right), 135.8(C 4), 135.1$ $\left(C 3^{\prime \prime \prime}\right), 134.5\left(C 4^{\prime}\right), 133.6\left(C 3^{\prime \prime}\right), 131.1\left(C 5^{\prime \prime \prime}\right), 130.3\left(C 5^{\prime \prime}\right)$, $129.9\left(C 2^{\prime \prime}\right), 128.7\left(C 6^{\prime \prime}\right), 127.0(C 3(5)), 126.9\left(C 4^{\prime \prime}\right), 126.2$ $\left(C 4^{\prime \prime \prime}\right), 125.5\left(C 2^{\prime \prime \prime}\right), 124.2\left(C 6^{\prime \prime \prime}\right), 122.4\left(C 5^{\prime}\right), 121.6\left(C 3^{\prime}\right)$. MS (CI, m/z): 396/394/392 (1/8/11, M+1), 317/315/313 (30/ 86/100), 278 (9), 146 (9). Anal. Calcd for $\mathrm{C}_{22} \mathrm{H}_{15} \mathrm{Cl}_{2} \mathrm{~N}_{3}$. $1 / 2 \mathrm{H}_{2} \mathrm{O}$ : C, 65.85; H, 4.02; N, 10.47. Found: C, 65.66; H, 3.87; N, 10.12 .

\subsection{7. $\mathrm{N}$-[3,5-Bis(trans-2-phenylvinyl)pyridin-2-yl]- pyridinium aminide $(\mathbf{6 g})$}

Yellow oil (319 mg, 85\%), mp hydrobromide $187-189^{\circ} \mathrm{C}$ (yellowish solid, ethanol); IR $(\mathrm{KBr}) \nu_{\max }\left(\mathrm{cm}^{-1}\right): 1619$, 1584, 1451, 1388, 1148, 957, 741, 691; ${ }^{1} \mathrm{H}$ NMR (300 MHz, $\left.\mathrm{CD}_{3} \mathrm{OD}\right): \delta 8.81(2 \mathrm{H}, \mathrm{dd}, J=6.9$ and $1.3 \mathrm{~Hz}, H 2(6)), 8.27$ $(1 \mathrm{H}, \mathrm{tt}, J=7.7$ and $1.3 \mathrm{~Hz}, H 4), 8.10\left(1 \mathrm{H}, \mathrm{d}, J=2.1 \mathrm{~Hz}, H 6^{\prime}\right)$, 
$7.96(2 \mathrm{H}, \mathrm{dd}, J=7.7$ and $6.9 \mathrm{~Hz}, H 3(5)), 7.75(1 \mathrm{H}, \mathrm{d}, J=$ $\left.2.1 \mathrm{~Hz}, H 4^{\prime}\right), 7.62\left(2 \mathrm{H}, \mathrm{m}, H 2^{\prime \prime}\left(6^{\prime \prime}\right)\right), 7.50\left(2 \mathrm{H}, \mathrm{m}, H 2^{\prime \prime \prime}\left(6^{\prime \prime \prime}\right)\right)$, $7.49\left(1 \mathrm{H}, \mathrm{d}, J=16.3 \mathrm{~Hz}, H_{\alpha^{\prime \prime}}\right), 7.33\left(1 \mathrm{H}, \mathrm{d}, J=16.3 \mathrm{~Hz}, H_{\beta^{\prime \prime}}\right)$, $7.34\left(4 \mathrm{H}, \mathrm{m}, H 3^{\prime \prime}\left(5^{\prime \prime}\right)\right.$ and $\left.H 3^{\prime \prime \prime}\left(5^{\prime \prime \prime}\right)\right), 7.25(1 \mathrm{H}, \mathrm{tt}, J=7.3$ and $\left.1.3 \mathrm{~Hz}, H 4^{\prime \prime}\right), 7.20\left(1 \mathrm{H}, \mathrm{tt}, J=7.3\right.$ and $\left.1.3 \mathrm{~Hz}, H 4^{\prime \prime \prime}\right), 7.05(2 \mathrm{H}$, m, $H_{\beta^{\prime \prime \prime}}$ and $\left.H_{\alpha^{\prime \prime \prime}}\right) ;{ }^{13} \mathrm{C}$ NMR $\left(75 \mathrm{MHz}, \mathrm{CD}_{3} \mathrm{OD}\right): \delta 159.8\left(C 2^{\prime}\right)$, 145.9 (C2(6)), $144.4\left(C 4^{\prime}\right), 140.6(C 4), 131.5\left(C_{\beta^{\prime \prime}}\right), 131.3$ $\left(C 1^{\prime \prime}\right), 131.1\left(C 6^{\prime}\right), 131.0\left(C 1^{\prime \prime \prime}\right), 129.4\left(C 3^{\prime \prime \prime}\left(5^{\prime \prime \prime}\right)\right.$ or $\left.C 3^{\prime \prime}\left(5^{\prime \prime}\right)\right)$, $129.3\left(C 3^{\prime \prime \prime}\left(5^{\prime \prime \prime}\right)\right.$ or $\left.C 3^{\prime \prime}\left(5^{\prime \prime}\right)\right)$, $128.8(C 3(5)), 128.4\left(C 4^{\prime \prime}\right), 127.8$ $\left(C 4^{\prime \prime \prime}\right), 127.4\left(C 2^{\prime \prime}\left(6^{\prime \prime}\right)\right), 126.9\left(C 2^{\prime \prime \prime}\left(6^{\prime \prime \prime}\right)\right), 126.5\left(C_{\alpha^{\prime \prime \prime}}\right.$ or $\left.C_{\beta^{\prime \prime \prime}}\right)$, $125.8\left(C_{\beta^{\prime \prime \prime}}\right.$ or $\left.C_{\alpha^{\prime \prime \prime}}\right), 124.2\left(C 5^{\prime}\right), 124.0\left(C_{\alpha^{\prime \prime}}\right), 121.4\left(C 3^{\prime}\right)$. MS (CI, $m / z): 376$ (5, M+1), 375 (9), 374 (12), 299 (59), 279 (9), 101 (33), 80 (100). Anal. Calcd for $\mathrm{C}_{26} \mathrm{H}_{21} \mathrm{~N}_{3} \cdot 2 \mathrm{HBr}$ : C, 58.12; $\mathrm{H}, 4.31$; N, 7.82. Found: C, 57.75; H, 4.32; N, 7.88.

\subsubsection{N-[3,5-Bis(pyridin-3-yl)pyridin-2-yl]pyridinium aminide (6h) and $N$-[5-bromo-3-(pyridin-3-yl)-pyridin- 2-yl]pyridinium aminide $(\mathbf{8})^{10 b}$}

Starting from dibromoaminide 2d and following general procedure A, two main products were obtained and separated by flash chromatography:

$\mathrm{N}$-[3,5-Bis(pyridin-3-yl)pyridin-2-yl]pyridinium aminide $(\boldsymbol{6 h})$ : orange oil $(59 \mathrm{mg}, 18 \%)$; IR $(\mathrm{KBr}) \nu_{\max }\left(\mathrm{cm}^{-1}\right): 1598$, 1446, 1402, 1314, 1146, 806, 712; ${ }^{1} \mathrm{H}$ NMR $(300 \mathrm{MHz}$, $\left.\mathrm{CD}_{3} \mathrm{OD}\right): \delta 8.95\left(1 \mathrm{H}\right.$, br d, $\left.J=2.2 \mathrm{~Hz}, H 2^{\prime \prime}\right), 8.75(3 \mathrm{H}, \mathrm{m}$, $H 2^{\prime \prime \prime}$ and $\left.H 2(6)\right), 8.49\left(1 \mathrm{H}, \mathrm{dd}, J=5.0\right.$ and $\left.1.6 \mathrm{~Hz}, H 6^{\prime \prime}\right)$, $8.42\left(1 \mathrm{H}, \mathrm{dd}, J=4.9\right.$ and $\left.1.6 \mathrm{~Hz}, H 6^{\prime \prime \prime}\right), 8.27(1 \mathrm{H}, \mathrm{dd}, J=8.0$, 2.2 and $\left.1.6 \mathrm{~Hz}, H 4^{\prime \prime}\right), 8.13(1 \mathrm{H}, \mathrm{tt}, J=7.6$ and $1.3 \mathrm{~Hz}, H 4)$, $8.04\left(1 \mathrm{H}, \mathrm{dd}, J=7.9,2.3\right.$ and $\left.1.6 \mathrm{~Hz}, H 4^{\prime \prime \prime}\right), 8.03(1 \mathrm{H}, \mathrm{d}$, $\left.J=2.5 \mathrm{~Hz}, H 6^{\prime}\right), 7.86(2 \mathrm{H}, \mathrm{dd}, J=7.6$ and $7.0 \mathrm{~Hz}, H 3(5))$, $7.72\left(1 \mathrm{H}, \mathrm{d}, J=2.5 \mathrm{~Hz}, H 4^{\prime}\right), 7.52(1 \mathrm{H}, \mathrm{dd}, J=8.0,5.0$ and $\left.0.7 \mathrm{~Hz}, H 5^{\prime \prime}\right), 7.47\left(1 \mathrm{H}, \mathrm{dd}, J=7.9,4.9\right.$ and $\left.0.7 \mathrm{~Hz}, H 5^{\prime \prime \prime}\right)$; ${ }^{13} \mathrm{C}$ NMR (75 MHz, CD $\left.{ }_{3} \mathrm{OD}\right): \delta 163.2\left(C 2^{\prime}\right), 150.1\left(C 2^{\prime \prime}\right)$, $147.7\left(C 6^{\prime \prime}\right), 147.0\left(C 6^{\prime \prime \prime}\right), 146.4\left(C 2^{\prime \prime \prime}\right), 145.5\left(C 6^{\prime}\right), 145.3$ $(C 2(6)), 138.8\left(C 4^{\prime \prime}\right), 138.5(C 4), 137.1\left(C 3^{\prime \prime}\right), 136.8\left(C 4^{\prime}\right)$, $136.0\left(C 3^{\prime \prime \prime}\right), 134.4\left(C 4^{\prime \prime \prime}\right), 128.3(C 3(5)), 125.2\left(C 5^{\prime \prime \prime}\right), 124.6$ $\left(C 5^{\prime \prime}\right), 120.8\left(C 5^{\prime}\right), 120.2\left(C 3^{\prime}\right)$. HRMS (FAB) calcd for $\mathrm{C}_{20} \mathrm{H}_{16} \mathrm{~N}_{5}:[\mathrm{M}+\mathrm{H}]^{+}$326.1406, found: 326.1402 .

$\mathrm{N}$-[5-bromo-3-(pyridin-3-yl)pyridin-2-yl] pyridinium aminide (8): orange oil $(157 \mathrm{mg}, 48 \%)$; IR $(\mathrm{KBr}) \nu_{\max }\left(\mathrm{cm}^{-1}\right)$ : $1705,1570,1524,1472,1385,1331,1259,1191,1146$, $1008,900,805,712 ;{ }^{1} \mathrm{H}$ NMR $\left(300 \mathrm{MHz}, \mathrm{CD}_{3} \mathrm{OD}\right): \delta 8.86$ $\left(1 \mathrm{H}, \mathrm{dd}, J=2.0\right.$ and $\left.0.7 \mathrm{~Hz}, H 2^{\prime \prime}\right), 8.70(2 \mathrm{H}, \mathrm{dd}, J=7.0$ and $1.3 \mathrm{~Hz}, H 2(6)), 8.48\left(1 \mathrm{H}, \mathrm{dd}, J=4.9\right.$ and $\left.1.6 \mathrm{~Hz}, H 6^{\prime \prime}\right), 8.17$ $\left(1 \mathrm{H}, \mathrm{dd}, J=8.0,2.0\right.$ and $\left.1.6 \mathrm{~Hz}, H 4^{\prime \prime}\right), 8.12(1 \mathrm{H}, \mathrm{tt}, J=7.8$ and $1.3 \mathrm{~Hz}, H 4), 7.84(2 \mathrm{H}$, dd, $J=7.8$ and $7.0 \mathrm{~Hz}, H 3(5))$, $7.71\left(1 \mathrm{H}, \mathrm{d}, J=2.4 \mathrm{~Hz}, H 6^{\prime}\right), 7.49(1 \mathrm{H}, \mathrm{dd}, J=8.0,4.9$ and $\left.0.7 \mathrm{~Hz}, H 5^{\prime \prime}\right), 7.46\left(1 \mathrm{H}, \mathrm{d}, J=2.4 \mathrm{~Hz}, H 4^{\prime}\right) ;{ }^{13} \mathrm{C}$ NMR $\left(75 \mathrm{MHz}, \mathrm{CD}_{3} \mathrm{OD}\right): \delta 161.7\left(C 2^{\prime}\right), 150.1\left(C 2^{\prime \prime}\right), 148.2\left(C 6^{\prime \prime}\right)$, $147.6\left(C 6^{\prime}\right), 145.5(C 2(6)), 140.5\left(C 4^{\prime}\right), 139.0(C 4), 138.7$ $\left(C 4^{\prime \prime}\right), 136.1\left(C 3^{\prime \prime}\right), 128.4(C 3(5)), 124.8\left(C 5^{\prime \prime}\right), 121.6\left(C 3^{\prime}\right)$, 105.2 $\left(C 5^{\prime}\right)$. HRMS (FAB) calcd for $\mathrm{C}_{15} \mathrm{H}_{12}^{79} \mathrm{BrN}_{4}$ : $[\mathrm{M}+\mathrm{H}]^{+}$ 327.0245, found: 327.0238 .

\subsection{9. $N$-(3,5-Diphenylpyrazin-2-yl)pyridinium aminide (7a)}

Red solid $\left(240 \mathrm{mg}, 74 \%\right.$, ethanol), mp $186-188^{\circ} \mathrm{C}$; IR $(\mathrm{KBr}) \nu_{\max }\left(\mathrm{cm}^{-1}\right): 1597,1577,1552,1508,1481,1414$,
1378, 1304， 1247，748，693，663; ${ }^{1} \mathrm{H}$ NMR $(300 \mathrm{MHz}$, $\left.\mathrm{CD}_{3} \mathrm{OD}\right): \delta 8.76(2 \mathrm{H}, \mathrm{dd}, J=6.9$ and $1.3 \mathrm{~Hz}, H 2(6)), 8.23$ $\left(2 \mathrm{H}, \mathrm{dd}, J=8.4\right.$ and $\left.1.5 \mathrm{~Hz}, H 2^{\prime \prime}\left(6^{\prime \prime}\right)\right), 8.14(1 \mathrm{H}, \mathrm{tt}, J=7.7$ and $1.3 \mathrm{~Hz}, H 4), 8.03\left(1 \mathrm{H}, \mathrm{s}, H 6^{\prime}\right), 7.88(4 \mathrm{H}, \mathrm{m}, H 3(5)$ and $\left.H 2^{\prime \prime \prime}\left(6^{\prime \prime \prime}\right)\right), 7.48\left(2 \mathrm{H}, \mathrm{tt}, J=7.2\right.$ and $\left.1.6 \mathrm{~Hz}, H 3^{\prime \prime}\left(5^{\prime \prime}\right)\right), 7.41(3 \mathrm{H}$, m, $H 3^{\prime \prime \prime}\left(5^{\prime \prime \prime}\right)$ and $\left.H 4^{\prime \prime}\right), 7.28\left(1 \mathrm{H}, \mathrm{tt}, J=7.3\right.$ and $\left.1.3 \mathrm{~Hz}, H 4^{\prime \prime \prime}\right)$; ${ }^{13} \mathrm{C}$ NMR (75 MHz, $\left.\mathrm{CD}_{3} \mathrm{OD}\right): \delta 158.8\left(C 2^{\prime}\right), 145.3(C 2(6))$, $141.9\left(C 3^{\prime}\right), 139.8\left(C 1^{\prime \prime}\right), 139.0\left(C 1^{\prime \prime \prime}\right), 138.7(C 4), 138.4$ $\left(C 5^{\prime}\right), 136.7\left(C 6^{\prime}\right), 130.0\left(C 2^{\prime \prime}\left(6^{\prime \prime}\right)\right), 129.3\left(C 3^{\prime \prime \prime}\left(5^{\prime \prime \prime}\right)\right), 129.0$ $\left(C 4^{\prime \prime}\right), 128.5\left(C 3^{\prime \prime}\left(5^{\prime \prime}\right)\right), 128.3(C 3(5)), 127.6\left(C 4^{\prime \prime \prime}\right), 125.5$ $\left(C 2^{\prime \prime \prime}\left(6^{\prime \prime \prime}\right)\right.$. MS (CI, m/z): 325 (100, M+1), 324 (40), 248 (51), 246 (30), 80 (63). Anal. Calcd for $\mathrm{C}_{21} \mathrm{H}_{16} \mathrm{~N}_{4} \cdot 1 / 4 \mathrm{H}_{2} \mathrm{O}$ : C, 76.68; H, 5.06; N, 17.04. Found: C, 76.97; H, 4.84; N, 17.46.

\subsubsection{N-[3,5-Bis(4-methoxyphenyl)pyrazin-2-yl]-} pyridinium aminide $(\mathbf{7 b})$

Red solid (331 mg, 86\%), mp hydrobromide $170-172{ }^{\circ} \mathrm{C}$ (yellowish solid, ethanol); IR $(\mathrm{KBr}) \nu_{\max }\left(\mathrm{cm}^{-1}\right)$ : 1606, 1509, 1489, 1435, 1397, 1371, 1244, 1176, 1144, 1031, 836; ${ }^{1} \mathrm{H}$ NMR $\left(300 \mathrm{MHz}, \mathrm{CD}_{3} \mathrm{OD}\right): \delta 8.66(2 \mathrm{H}, \mathrm{dd}, J=6.9$ and $1.2 \mathrm{~Hz}, H 2(6)), 8.18\left(2 \mathrm{H}, \mathrm{d}, J=8.9 \mathrm{~Hz}, H 2^{\prime \prime}\left(6^{\prime \prime}\right)\right), 8.01(1 \mathrm{H}$, tt, $J=7.8$ and $1.2 \mathrm{~Hz}, H 4), 7.86\left(1 \mathrm{H}, \mathrm{s}, H 6^{\prime}\right), 7.77(2 \mathrm{H}, \mathrm{d}, J=$ $\left.8.9 \mathrm{~Hz}, H 2^{\prime \prime \prime}\left(6^{\prime \prime \prime}\right)\right), 7.76(2 \mathrm{H}, \mathrm{dd}, J=7.8$ and $6.9 \mathrm{~Hz}, H 3(5))$, $6.97\left(2 \mathrm{H}, \mathrm{d}, J=8.9 \mathrm{~Hz}, H 3^{\prime \prime}\left(5^{\prime \prime}\right)\right), 6.93(2 \mathrm{H}, \mathrm{d}, J=8.9 \mathrm{~Hz}$, $\left.\mathrm{H}^{\prime \prime \prime}\left(5^{\prime \prime \prime}\right)\right), 3.83\left(3 \mathrm{H}, \mathrm{s}, \mathrm{OCH}_{3}\right), 3.79\left(3 \mathrm{H}, \mathrm{s}, \mathrm{OCH}_{3}\right) ;{ }^{13} \mathrm{C}$ NMR (75 MHz, $\left.\mathrm{CDCl}_{3}\right): \delta 160.8\left(C 4^{\prime \prime}\right), 160.0\left(C 4^{\prime \prime \prime}\right), 158.2$ $\left(C 2^{\prime}\right), 144.9(C 2(6)), 141.5\left(C 3^{\prime}\right), 138.5\left(C 5^{\prime}\right), 138.0(C 4)$, $135.2\left(C 6^{\prime}\right), 132.2\left(C 1^{\prime \prime}\right), 131.8\left(C 1^{\prime \prime \prime}\right), 131.5\left(C 2^{\prime \prime}\left(6^{\prime \prime}\right)\right)$, $128.1(C 3(5)), 126.8\left(C 2^{\prime \prime \prime}\left(6^{\prime \prime \prime}\right)\right), 114.7\left(C 3^{\prime \prime \prime}\left(5^{\prime \prime \prime}\right)\right), 113.9$ $\left(\mathrm{C}^{\prime \prime}\left(5^{\prime \prime}\right)\right), 55.7\left(\mathrm{OCH}_{3}\right)$. MS (CI, m/z): $385(43, \mathrm{M}+1), 384$ (24), 308 (100), 307 (26), 306 (24), 80 (64). Anal. Calcd for $\mathrm{C}_{23} \mathrm{H}_{20} \mathrm{~N}_{4} \mathrm{O}_{2} \cdot \mathrm{HBr} \cdot \mathrm{H}_{2} \mathrm{O}: \mathrm{C}, 57.15 ; \mathrm{H}, 4.80 ; \mathrm{N}, 11.59$. Found: C, 57.04; H, 4.80; N, 11.45.

\subsubsection{N-[3,5-Bis(4-acetylphenyl)pyrazin-2-yl]pyridinium aminide $(7 \mathrm{c})$}

Red solid (274 mg, 95\%, ethanol), mp 196-197 ${ }^{\circ} \mathrm{C}$; IR $(\mathrm{KBr}) \nu_{\max }\left(\mathrm{cm}^{-1}\right): 1666,1598,1489,1398,1269,1147$, 841; ${ }^{1} \mathrm{H}$ NMR $\left(300 \mathrm{MHz}, \mathrm{CD}_{3} \mathrm{OD}\right): \delta 8.79(2 \mathrm{H}, \mathrm{dd}, J=6.9$ and $1.3 \mathrm{~Hz}, H 2(6)), 8.52\left(2 \mathrm{H}, \mathrm{d}, J=8.6 \mathrm{~Hz}, H 2^{\prime \prime}\left(6^{\prime \prime}\right)\right), 8.23(1 \mathrm{H}$, s, $\left.H 6^{\prime}\right), 8.21(1 \mathrm{H}, \mathrm{tt}, J=7.6$ and $1.3 \mathrm{~Hz}, H 4), 8.12(2 \mathrm{H}, \mathrm{d}$, $\left.J=8.6 \mathrm{~Hz}, H 3^{\prime \prime}\left(5^{\prime \prime}\right)\right), 8.10\left(2 \mathrm{H}, \mathrm{d}, J=8.8 \mathrm{~Hz}, H 2^{\prime \prime \prime}\left(6^{\prime \prime \prime}\right)\right), 8.06$ $\left(2 \mathrm{H}, \mathrm{d}, J=8.8 \mathrm{~Hz}, H 3^{\prime \prime \prime}\left(5^{\prime \prime \prime}\right)\right), 7.93(2 \mathrm{H}, \mathrm{dd}, J=7.6$ and $6.9 \mathrm{~Hz}, \mathrm{H3}(5)), 2.70\left(3 \mathrm{H}, \mathrm{s}, \mathrm{COCH}_{3}\right), 2.65\left(3 \mathrm{H}, \mathrm{s}, \mathrm{COCH}_{3}\right)$; ${ }^{13} \mathrm{C}$ NMR $\left(75 \mathrm{MHz}, \mathrm{CDCl}_{3}\right)$ : $\delta 198.0(\mathrm{CO}), 197.7(\mathrm{CO})$, $157.3\left(C 2^{\prime}\right), 143.6\left(C 3^{\prime}\right), 142.9(C 2(6)), 142.7\left(C 1^{\prime \prime}\right), 139.3$ $\left(C 5^{\prime}\right), 137.9\left(C 6^{\prime}\right), 136.5\left(C 1^{\prime \prime \prime}\right), 136.4\left(C 4^{\prime \prime \prime}\right), 135.3\left(C 4^{\prime \prime}\right)$, $135.0(C 4), 129.7\left(C 2^{\prime \prime \prime}\left(6^{\prime \prime \prime}\right)\right), 129.1\left(C 3^{\prime \prime}\left(5^{\prime \prime}\right)\right), 128.0\left(C 3^{\prime \prime \prime}\left(5^{\prime \prime \prime}\right)\right.$ or $C 3(5)), 126.4\left(C 3(5)\right.$ or $\left.C 3^{\prime \prime \prime}\left(5^{\prime \prime \prime}\right)\right), 124.4\left(C 2^{\prime \prime}\left(6^{\prime \prime}\right)\right), 27.4$ $\left(\mathrm{CH}_{3}\right), 27.2\left(\mathrm{CH}_{3}\right)$. MS (CI, m/z): $409(7, \mathrm{M}+1), 316(17), 333$ (39), 332 (100), 80 (86). Anal. Calcd for $\mathrm{C}_{25} \mathrm{H}_{20} \mathrm{~N}_{4} \mathrm{O}_{2} \cdot 1 / 4 \mathrm{H}_{2} \mathrm{O}$ : C, 72.70; H, 5.01; N, 13.57. Found: C, 72.61; H, 4.95; N, 13.67.

\subsubsection{N-[3,5-Bis(trans-2-phenylvinyl)pyrazin-2-yl]-} pyridinium aminide $(\mathbf{7 d})$

Red solid (331 mg, 88\%), mp hydrobromide $207-209^{\circ} \mathrm{C}$ (yellowish solid, ethanol); IR (KBr) $\nu_{\max }\left(\mathrm{cm}^{-1}\right)$ : 1733, 
1595, 1547, 1489, 1372, 1145, 965, 744, 691; ${ }^{1} \mathrm{H}$ NMR $\left(500 \mathrm{MHz}, \mathrm{CD}_{3} \mathrm{OD}\right): \delta 8.80(2 \mathrm{H}, \mathrm{dd}, J=6.9$ and $1.3 \mathrm{~Hz}$, $H 2(6)), 8.15(1 \mathrm{H}, \mathrm{tt}, J=7.7$ and $1.3 \mathrm{~Hz}, H 4), 7.92(1 \mathrm{H}, \mathrm{d}$, $\left.J=16.1 \mathrm{~Hz}, H_{\beta^{\prime \prime}}\right), 7.89(2 \mathrm{H}, \mathrm{dd}, J=7.7$ and $6.9 \mathrm{~Hz}, H 3(5))$, $7.71\left(1 \mathrm{H}, \mathrm{d}, J=16.1 \mathrm{~Hz}, H_{\alpha^{\prime \prime}}\right), 7.69(2 \mathrm{H}, \mathrm{dd}, J=8.5$ and $\left.1.3 \mathrm{~Hz}, H 2^{\prime \prime}\left(6^{\prime \prime}\right)\right), 7.64\left(1 \mathrm{H}, \mathrm{s}, H 6^{\prime}\right), 7.56(2 \mathrm{H}, \mathrm{dd}, J=8.6$ and $\left.1.3 \mathrm{~Hz}, \quad H 2^{\prime \prime \prime}\left(6^{\prime \prime \prime}\right)\right), \quad 7.41 \quad(2 \mathrm{H}, \quad \mathrm{dd}, \quad J=8.5$ and $7.3 \mathrm{~Hz}$, $\left.H 3^{\prime \prime}\left(5^{\prime \prime}\right)\right), 7.39\left(1 \mathrm{H}, \mathrm{d}, J=16.1 \mathrm{~Hz}, H_{\beta^{\prime \prime \prime}}\right), 7.35(2 \mathrm{H}, \mathrm{dd}$, $J=8.6$ and $\left.7.3 \mathrm{~Hz}, H 3^{\prime \prime \prime}\left(5^{\prime \prime \prime}\right)\right), 7.31 \quad(1 \mathrm{H}, \mathrm{tt}, J=7.3$ and $\left.1.3 \mathrm{~Hz}, H 4^{\prime \prime}\right), 7.24\left(1 \mathrm{H}, \mathrm{tt}, J=7.3\right.$ and $\left.1.3 \mathrm{~Hz}, H 4^{\prime \prime \prime}\right), 7.09$ $\left(1 \mathrm{H}, \mathrm{d}, J=16.1 \mathrm{~Hz}, H_{\alpha^{\prime \prime \prime}}\right) ;{ }^{13} \mathrm{C}$ NMR $\left(125 \mathrm{MHz}, \mathrm{CD}_{3} \mathrm{OD}\right)$ : o $159.4\left(C 2^{\prime}\right), 145.7(C 2(6)), 140.1\left(C 3^{\prime}\right), 140.0\left(C 6^{\prime}\right), 139.5$ $\left(C 1^{\prime \prime \prime}\right), 139.3(C 4), 139.1\left(C 1^{\prime \prime}\right), 137.9\left(C 5^{\prime}\right), 134.9\left(C_{\beta^{\prime \prime}}\right)$, $129.9\left(C 3^{\prime \prime}\left(5^{\prime \prime}\right)\right), \quad 129.8 \quad\left(C 3^{\prime \prime \prime}\left(5^{\prime \prime \prime}\right)\right), \quad 129.3 \quad\left(C 4^{\prime \prime}\right), \quad 128.8$ $(C 3(5)), 128.3\left(C 2^{\prime \prime}\left(6^{\prime \prime}\right)\right), 128.2\left(C 4^{\prime \prime \prime}\right), 127.5\left(C 2^{\prime \prime \prime}\left(6^{\prime \prime \prime}\right)\right.$ and $\left.C_{\beta^{\prime \prime \prime}}\right), 126.6\left(C_{\alpha^{\prime \prime \prime}}\right), 124.7\left(C_{\alpha^{\prime \prime}}\right) . \mathrm{MS}(\mathrm{CI}, \mathrm{m} / \mathrm{z}): 377(50$, $\mathrm{M}+1$ ), 376 (21), 300 (100), 299 (29), 80 (20). Anal. Calcd for $\mathrm{C}_{25} \mathrm{H}_{20} \mathrm{~N}_{4} \cdot \mathrm{HBr}$ : $\mathrm{C}, 65.65 ; \mathrm{H}, 4.63 ; \mathrm{N}, 12.25$. Found: C, $65.26 ; \mathrm{H}, 4.79 ; \mathrm{N}, 12.17$.

\subsubsection{N-[3,5-Bis(thiophen-3-yl)pyrazin-2-yl]pyridinium aminide (7e)}

Red solid (328 mg, 98\%), mp 82-84 ${ }^{\circ} \mathrm{C}, \mathrm{mp}$ hydrobromide 134-137 ${ }^{\circ} \mathrm{C}$ (yellow solid); IR $(\mathrm{KBr}) \nu_{\max }\left(\mathrm{cm}^{-1}\right)$ : 3108 , $1529,1488,1426,1383,1367,1244,1178,1145,768 ;{ }^{1} \mathrm{H}$ NMR (500 MHz, $\left.\left(\mathrm{CD}_{3}\right)_{2} \mathrm{CO}\right): \delta 8.94\left(3 \mathrm{H}, \mathrm{m}, H 2(6)\right.$ and $\left.H 2^{\prime \prime}\right)$, $8.25\left(1 \mathrm{H}, \mathrm{dd}, J=5.1\right.$ and $\left.1.1 \mathrm{~Hz}, H 4^{\prime \prime}\right), 8.04\left(1 \mathrm{H}, \mathrm{s}, H 6^{\prime}\right), 7.97$ $(1 \mathrm{H}, \quad \mathrm{tt}, \quad J=7.7$ and $1.2 \mathrm{~Hz}, H 4), \quad 7.80 \quad(2 \mathrm{H}, \quad \mathrm{dd}, \quad J=$ 7.7 and $7.0 \mathrm{~Hz}, H 3(5)), 7.76\left(1 \mathrm{H}, \mathrm{dd}, J=3.0\right.$ and $\left.1.2 \mathrm{~Hz}, H 2^{\prime \prime \prime}\right)$, $7.66\left(1 \mathrm{H}, \mathrm{dd}, J=5.0\right.$ and $\left.1.2 \mathrm{~Hz}, H 4^{\prime \prime \prime}\right), 7.46(1 \mathrm{H}, \mathrm{dd}, J=5.0$ and $\left.3.0 \mathrm{~Hz}, H 5^{\prime \prime \prime}\right), 7.41\left(1 \mathrm{H}, \mathrm{dd}, J=5.1\right.$ and $\left.3.0 \mathrm{~Hz}, H 5^{\prime \prime}\right) ;{ }^{13} \mathrm{C}$ NMR (75 MHz, $\left.\left(\mathrm{CD}_{3}\right)_{2} \mathrm{CO}\right): \delta 157.1\left(C 2^{\prime}\right), 143.9(C 2(6))$, $142.3\left(C 5^{\prime}\right), 141.4\left(C 3^{\prime}\right), 136.0\left(C 6^{\prime}\right), 135.9(C 4), 135.4\left(C 3^{\prime \prime}\right)$, $134.6\left(C 3^{\prime \prime \prime}\right), 129.8\left(C 4^{\prime \prime}\right), 127.3\left(C 2^{\prime \prime}\right), 127.2(C 3(5)), 126.5$ $\left(C 5^{\prime \prime \prime}\right), 126.0\left(C 4^{\prime \prime \prime}\right), 123.8\left(C 5^{\prime \prime}\right), 118.5\left(C 5^{\prime \prime \prime}\right)$. MS (EI, $\left.\mathrm{m} / \mathrm{z}\right)$ : $336\left(64, \mathrm{M}^{+}\right), 257$ (84), 121 (100), 79 (58). HRMS (ESI-TOF, $\left.\mathrm{CH}_{3} \mathrm{OH}\right)$ calcd for $\mathrm{C}_{17} \mathrm{H}_{13} \mathrm{~N}_{4} \mathrm{~S}_{2}:[\mathrm{M}+\mathrm{H}]^{+} 337.05761$, found: 337.05976 .

\subsubsection{N-[3,5-Bis(benzo[b]thiophen-3-yl)pyraz-2-yl]- pyridinium aminide $(\mathbf{7 f})$}

Orange solid $(387 \mathrm{mg}, 89 \%), \mathrm{mp} 104-106{ }^{\circ} \mathrm{C}$; IR (KBr) $\nu_{\max }\left(\mathrm{cm}^{-1}\right): 1555,1515,1486,1408,1208,1144,862,784$, 758, 732, 700; ${ }^{1} \mathrm{H}$ NMR $\left(500 \mathrm{MHz},\left(\mathrm{CD}_{3}\right)_{2} \mathrm{CO}\right): \delta 9.28(1 \mathrm{H}$, s, $\left.H 2^{\prime \prime}\right), 9.08\left(1 \mathrm{H}\right.$, ddd, $J=8.2,1.3$ and $\left.0.7 \mathrm{~Hz}, H 4^{\prime \prime}\right), 8.94$ $(2 \mathrm{H}, \mathrm{dd}, J=7.0$ and $1.3 \mathrm{~Hz}, H 2(6)), 8.56(1 \mathrm{H}, \mathrm{ddd}, J=7.4$, 1.4 and $\left.0.7 \mathrm{~Hz}, H 4^{\prime \prime \prime}\right), 8.10\left(1 \mathrm{H}, \mathrm{s}, H 6^{\prime}\right), 8.06(1 \mathrm{H}, \mathrm{tt}, J=7.7$ and $1.3 \mathrm{~Hz}, H 4), 7.97\left(2 \mathrm{H}, \mathrm{m}, H 7^{\prime \prime \prime}\right.$ and $\left.H 7^{\prime \prime}\right), 7.87(2 \mathrm{H}, \mathrm{dd}$, $J=7.7$ and $7.0 \mathrm{~Hz}, H 3(5)), 7.82\left(1 \mathrm{H}, \mathrm{s}, H 2^{\prime \prime \prime}\right), 7.34(4 \mathrm{H}, \mathrm{m}$, $H 5^{\prime \prime \prime}, H 5^{\prime \prime}, H 6^{\prime \prime \prime}$ and $\left.H 6^{\prime \prime}\right) ;{ }^{13} \mathrm{C}$ NMR $\left(125 \mathrm{MHz},\left(\mathrm{CD}_{3}\right)_{2} \mathrm{CO}\right)$ : $\delta 158.0\left(C 2^{\prime}\right), 144.4\left(C 2(6), 141.7\left(C 7 \mathrm{a}^{\prime \prime \prime}\right), 140.5\left(C 7 \mathrm{a}^{\prime \prime}\right)\right.$, $140.1\left(C 3 \mathrm{a}^{\prime \prime}\right), 138.7\left(C 3 \mathrm{a}^{\prime \prime \prime}\right), 138.2\left(C 6^{\prime}\right), 136.8(C 4), 136.3$ $\left(C 3^{\prime}\right)$, $134.6\left(C 3^{\prime \prime \prime}\right), 133.8\left(C 5^{\prime}\right), 132.0\left(C 3^{\prime \prime}\right), 130.5\left(C 2^{\prime \prime}\right)$, $127.6(C 3(5)), 127.3\left(C 4^{\prime \prime}\right), 125.6\left(C 4^{\prime \prime \prime}\right), 125.2\left(C 6^{\prime \prime \prime}\right), 124.9$ $\left(C 5^{\prime \prime \prime}\right.$ and $\left.C 5^{\prime \prime}\right), 124.8\left(C 6^{\prime \prime}\right), 123.5\left(C 7^{\prime \prime}\right), 123.1\left(C 7^{\prime \prime \prime}\right)$, $122.0\left(C 2^{\prime \prime \prime}\right)$. MS (ESI, $\left.m / z\right): 437$ (100, M+1), 358 (80), 277
(41), 245 (56). HRMS (ESI-TOF, $\mathrm{CH}_{3} \mathrm{OH}$ ) calcd for $\mathrm{C}_{25} \mathrm{H}_{17} \mathrm{~N}_{4} \mathrm{~S}_{2}:[\mathrm{M}+\mathrm{H}]^{+}$437.08891, found: 437.09109 .

\subsubsection{N-[3,5-Bis(pyridin-3-yl)pyrazin-2-yl]pyridinium aminide $(7 \mathrm{~g})$}

Orange solid $(212 \mathrm{mg}, 65 \%$, ethanol/diethyl ether), $\mathrm{mp}$ 220-221 ${ }^{\circ} \mathrm{C}$; IR $(\mathrm{KBr}) \nu_{\max }\left(\mathrm{cm}^{-1}\right): 1555,1496,1445$, $1407,1365,1309,1270,1147,709 ;{ }^{1} \mathrm{H}$ NMR $(300 \mathrm{MHz}$, $\left.\mathrm{CD}_{3} \mathrm{OD}\right): \delta 9.55\left(1 \mathrm{H}, \mathrm{d}, J=2.1 \mathrm{~Hz}, H 2^{\prime \prime}\right), 9.09(1 \mathrm{H}, \mathrm{d}, J=$ $\left.2.3 \mathrm{~Hz}, H 2^{\prime \prime \prime}\right), 8.85\left(1 \mathrm{H}\right.$, ap dt, $J=8.1$ and $\left.2.1 \mathrm{~Hz}, H 4^{\prime \prime}\right), 8.80$ $(2 \mathrm{H}, \mathrm{dd}, J=6.9$ and $1.3 \mathrm{~Hz}, H 2(6)), 8.55(1 \mathrm{H}, \mathrm{dd}, J=4.9$ and $\left.1.8 \mathrm{~Hz}, H 6^{\prime \prime}\right), 8.43\left(1 \mathrm{H}, \mathrm{dd}, J=4.9\right.$ and $\left.1.8 \mathrm{~Hz}, H 6^{\prime \prime \prime}\right), 8.37$ $\left(1 \mathrm{H}\right.$, ap dt, $J=8.1$ and $\left.2.3 \mathrm{~Hz}, H 4^{\prime \prime \prime}\right), 8.19(1 \mathrm{H}, \mathrm{tt}, J=7.5$ and $1.3 \mathrm{~Hz}, H 4), 8.18\left(1 \mathrm{H}, \mathrm{s}, H 6^{\prime}\right), 7.92(2 \mathrm{H}, \mathrm{dd}, J=7.5$ and $6.9 \mathrm{~Hz}, H 3(5)), 7.55\left(1 \mathrm{H}, \mathrm{dd}, J=8.1\right.$ and $\left.4.9 \mathrm{~Hz}, H 5^{\prime \prime}\right), 7.48$ $\left(1 \mathrm{H}, \mathrm{dd}, J=8.1\right.$ and $\left.4.9 \mathrm{~Hz}, H 5^{\prime \prime \prime}\right) ;{ }^{13} \mathrm{C}$ NMR $(75 \mathrm{MHz}$, $\left.\mathrm{CD}_{3} \mathrm{OD}\right): \delta 159.4\left(C 2^{\prime}\right), 150.2\left(C 2^{\prime \prime}\right), 148.5\left(C 6^{\prime \prime \prime}\right), 147.4$ $\left(C 6^{\prime \prime}\right), 145.8\left(C 2^{\prime \prime \prime}\right), 145.2(C 2(6)), 139.2(C 4), 138.5\left(C 6^{\prime}\right)$, $138.4\left(C 4^{\prime \prime}\right), 137.7\left(C 3^{\prime}\right), 136.1\left(C 3^{\prime \prime}\right), 135.3\left(C 3^{\prime \prime \prime}\right), 134.6$ (C5'), 133.3 (C4'"'), $128.3(C 3(5)), 125.0\left(C 5^{\prime \prime \prime}\right), 124.2\left(C 5^{\prime \prime}\right)$. MS (CI, m/z): 327 (7, M+1), 278 (10), 250 (60), 173 (10), 124 (10), 108 (17), 80 (100). Anal. Calcd for $\mathrm{C}_{19} \mathrm{H}_{14} \mathrm{~N}_{6}$. $1 / 2 \mathrm{H}_{2} \mathrm{O}: \mathrm{C}, 68.05 ; \mathrm{H}, 4.51 ; \mathrm{N}, 25.06$. Found: $\mathrm{C}, 67.78 ; \mathrm{H}$, 4.42; N, 24.77.

5.4. Synthesis of aminides 10: general procedure for the selective coupling over $N$-(3,5-dibromopyrazin-2-yl)pyridinium aminide $(\mathbf{2 e})$

Dibromoaminide $2 \mathrm{e}^{8 \mathrm{~b}, \mathrm{c}, \mathrm{h}}(330 \mathrm{mg}, 1 \mathrm{mmol})$ and the corresponding boronic acid $(1.1 \mathrm{mmol})$ were dissolved in a toluene/ethanol mixture $(4: 1,15 \mathrm{~mL}) . \mathrm{K}_{2} \mathrm{CO}_{3}(10 \mathrm{mmol})$ was added followed by $\mathrm{Pd}\left(\mathrm{PPh}_{3}\right)_{4}(5 \mathrm{~mol} \%)$. After the addition, the mixture was kept under argon with vigorous stirring for $5 \mathrm{~min}$, and then heated under reflux for the reaction time indicated in Table 3 (i.e., until starting material was not detected by TLC). The system was allowed to reach room temperature and the mixture was filtered through Celite or silica gel. The residue was washed with acetonitrile until colour was no longer observed in the filtrates, which were combined and evaporated to dryness. The residue was purified by flash chromatography on a silica gel column, using ethanol as eluent. Finally, the bromo compounds $\mathbf{1 0}$ were, if possible, recrystallized from a suitable solvent.

\subsubsection{N-(5-Bromo-3-phenylpyrazin-2-yl)pyridinium aminide (10a)}

See Ref. 10b.

\subsubsection{N-[5-Bromo-3-(4-methoxyphenyl)pyrazin- \\ 2-yl]pyridinium aminide $(\mathbf{1 0 b})$}

Orange solid (254 mg, 71\%, ethyl acetate), mp 182$183{ }^{\circ} \mathrm{C}$; IR (KBr) $\nu_{\max }\left(\mathrm{cm}^{-1}\right): 1483,1417,1375,1270$, $1214,969,744,671 ;{ }^{1} \mathrm{H}$ NMR $\left(300 \mathrm{MHz}, \mathrm{CD}_{3} \mathrm{OD}\right): \delta 8.79$ $(2 \mathrm{H}, \mathrm{dd}, J=6.9$ and $1.3 \mathrm{~Hz}, H 2(6)), 8.28(1 \mathrm{H}, \mathrm{tt}, J=7.8$ and $1.3 \mathrm{~Hz}, H 4), 8.12\left(2 \mathrm{H}, \mathrm{d}, J=8.9 \mathrm{~Hz}, H 2^{\prime \prime}\left(6^{\prime \prime}\right)\right), 7.96(2 \mathrm{H}$, dd, $J=7.8$ and $6.9 \mathrm{~Hz}, H 3(5)), 7.64\left(1 \mathrm{H}, \mathrm{s}, H 6^{\prime}\right), 7.03(2 \mathrm{H}$, 
$\left.\mathrm{d}, J=8.9 \mathrm{~Hz}, \mathrm{H3}^{\prime \prime}\left(5^{\prime \prime}\right)\right), 3.89\left(3 \mathrm{H}, \mathrm{s}, \mathrm{CH}_{3}\right) ;{ }^{13} \mathrm{C} \mathrm{NMR}(75 \mathrm{MHz}$, $\left.\mathrm{CDCl}_{3}\right): \delta 160.0\left(C 4^{\prime \prime}\right), 156.6\left(C 2^{\prime}\right), 142.6(C 2(6)), 142.1$ $\left(C 3^{\prime}\right), 139.5\left(C 6^{\prime}\right), 134.2(C 4), 131.0\left(C 2^{\prime \prime}\left(6^{\prime \prime}\right)\right), 130.2\left(C 1^{\prime \prime}\right)$, $126.3(C 3(5)), 122.0\left(C 5^{\prime}\right), 113.4\left(C 3^{\prime \prime}\left(5^{\prime \prime}\right)\right), 55.7\left(C \mathrm{H}_{3}\right)$. Anal. Calcd for $\mathrm{C}_{16} \mathrm{H}_{13} \mathrm{BrN}_{4} \mathrm{O}$ : C, 53.80; H, 3.67; N, 15.68. Found: C, 53.56; H, 3.84; N, 15.56.

\subsubsection{N-[5-Bromo-3-(3,5-dimethylphenyl)pyrazin-2-yl]- pyridinium aminide $(\mathbf{1 0 c})$}

Brown solid (301 mg, 85\%), mp 82-84 ${ }^{\circ} \mathrm{C}$ (mp hydrobromide $130-132{ }^{\circ} \mathrm{C}$, yellowish solid, ethanol); IR (KBr) $\nu_{\max }$ $\left(\mathrm{cm}^{-1}\right)$ : 2913, 1600, 1521, 1487, 1398, 1212, 1142, 852, 765, 697, 671; ${ }^{1} \mathrm{H}$ NMR $\left(300 \mathrm{MHz},\left(\mathrm{CD}_{3}\right)_{2} \mathrm{CO}\right): \delta 8.80(2 \mathrm{H}$, $\mathrm{dd}, J=7.0$ and $1.3 \mathrm{~Hz}, H 2(6)), 8.07(1 \mathrm{H}, \mathrm{tt}, J=7.7$ and $1.3 \mathrm{~Hz}, H 4), 8.02\left(2 \mathrm{H}\right.$, br s, $\left.H 2^{\prime \prime}\left(6^{\prime \prime}\right)\right), 7.86(2 \mathrm{H}$, dd, $J=7.7$ and $7.0 \mathrm{~Hz}, H 3(5)), 7.51\left(1 \mathrm{H}, \mathrm{s}, H 6^{\prime}\right), 6.97\left(1 \mathrm{H}\right.$, br s, $\left.H 4^{\prime \prime}\right)$, $2.30\left(6 \mathrm{H}, \mathrm{s}, \mathrm{CH}_{3}\right) ;{ }^{13} \mathrm{C} \mathrm{NMR}\left(75 \mathrm{MHz},\left(\mathrm{CD}_{3}\right)_{2} \mathrm{CO}\right): \delta 158.6$ $\left(C 2^{\prime}\right), 144.4(C 2(6)), 141.0\left(C 6^{\prime}\right), 138.6\left(C 3^{\prime}\right), 137.2(C 4)$, 137,1 $\left(C 1^{\prime \prime}\right), 130.4\left(C 3^{\prime \prime}\left(5^{\prime \prime}\right)\right), 127.8(C 3(5)), 127.8\left(C 2^{\prime \prime}\left(6^{\prime \prime}\right)\right)$, $123.3\left(\mathrm{C4}^{\prime \prime}\right), 120.5\left(\mathrm{C5}^{\prime}\right), 21.5\left(\mathrm{CH}_{3}\right)$. MS (CI, $\left.\mathrm{m} / \mathrm{z}\right): 357 / 355$ (99/100, M+1), 278/276 (41/41). Anal. Calcd for $\mathrm{C}_{17} \mathrm{H}_{15} \mathrm{BrN}_{4}$ : C, 57.48; H, 4.26; N, 15.77. Found: C, 57.80; $\mathrm{H}, 4.46 ; \mathrm{N}, 15.50$.

\subsubsection{N-[3-(4-Acetylphenyl)-5-bromopyrazin-2-yl]- pyridinium aminide (10d)}

Orange solid (240 mg, 65\%, ethyl acetate), mp 190$192{ }^{\circ} \mathrm{C}$; IR (KBr) $\nu_{\max }\left(\mathrm{cm}^{-1}\right): 1664,1602,1473,1428$, 1399, 1359, 1274, 1162, 840, 759, 677; ${ }^{1} \mathrm{H}$ NMR $(300 \mathrm{MHz}$, $\left.\mathrm{CD}_{3} \mathrm{OD}\right): \delta 8.73(2 \mathrm{H}, \mathrm{dd}, J=6.9$ and $1.3 \mathrm{~Hz}, H 2(6)), 8.38$ $\left(2 \mathrm{H}, \mathrm{d}, J=8.6 \mathrm{~Hz}, H 2^{\prime \prime}\left(6^{\prime \prime}\right)\right), 8.17(1 \mathrm{H}, \mathrm{tt}, J=7.7$ and $1.3 \mathrm{~Hz}$, $H 4), 8.06\left(2 \mathrm{H}, \mathrm{d}, J=8.6 \mathrm{~Hz}, H 3^{\prime \prime}\left(5^{\prime \prime}\right)\right), 7.88(2 \mathrm{H}, \mathrm{dd}, J=7.7$ and $6.9 \mathrm{~Hz}, H 3(5)), 7.63\left(1 \mathrm{H}, \mathrm{s}, \mathrm{H6}^{\prime}\right), 2.67\left(3 \mathrm{H}, \mathrm{s}, \mathrm{CH}_{3}\right)$; ${ }^{13} \mathrm{C}$ NMR $\left(75 \mathrm{MHz}, \mathrm{CDCl}_{3}\right): \delta 198.1(C \mathrm{O}), 157.1\left(C 2^{\prime}\right)$, $142.8(C 2(6)), 142.3\left(C 1^{\prime \prime}\right), 141.6\left(C 6^{\prime}\right), 139.8\left(C 3^{\prime}\right), 136.5$ $\left(C 4^{\prime \prime}\right), 135.0(C 4), 129.5\left(C 2^{\prime \prime}\left(6^{\prime \prime}\right)\right), 128.0\left(C 3^{\prime \prime}\left(5^{\prime \prime}\right)\right), 126.5$ $(C 3(5)), \quad 121.8 \quad\left(C 5^{\prime}\right), 27.2 \quad\left(C_{3}\right)$. Anal. Calcd for $\mathrm{C}_{17} \mathrm{H}_{13} \mathrm{BrN}_{4} \mathrm{O}$ : C, 55.30; H, 3.55; N, 15.17. Found: C, 54.99; H, 3.71; N, 14.83 .

\subsubsection{N-[5-Bromo-3-(trans-2-phenylvinyl)pyrazin-2-yl]- pyridinium aminide $(\mathbf{1 0 e})$}

Orange solid (177 mg, 50\%, ethyl acetate), mp 200$202^{\circ} \mathrm{C}$; IR (KBr) $\nu_{\max }\left(\mathrm{cm}^{-1}\right): 1602,1507,1471,1398$, $1365,1243,1172,1015,842,814,654 ;{ }^{1} \mathrm{H}$ NMR $(300 \mathrm{MHz}$, $\left.\mathrm{CD}_{3} \mathrm{OD}\right): \delta 8.77(2 \mathrm{H}$, dd, $J=6.9$ and $1.3 \mathrm{~Hz}, H 2(6)), 8.18$ $(1 \mathrm{H}, \mathrm{tt}, J=7.7$ and $1.3 \mathrm{~Hz}, H 4), 7.90(2 \mathrm{H}, \mathrm{dd}, J=7.7$ and $6.9 \mathrm{~Hz}, H 3(5)), 7.80\left(1 \mathrm{H}, \mathrm{d}, J=16.0 \mathrm{~Hz}, H_{\beta}\right), 7.65(2 \mathrm{H}, \mathrm{m}$, $\left.H 2^{\prime \prime}\left(6^{\prime \prime}\right)\right), 7.55\left(1 \mathrm{H}, \mathrm{d}, J=16.0 \mathrm{~Hz}, H_{\alpha}\right), 7.53\left(1 \mathrm{H}, \mathrm{s}, H 6^{\prime}\right)$, $7.39\left(2 \mathrm{H}, \mathrm{m}, H 3^{\prime \prime}\left(5^{\prime \prime}\right)\right), 7.30(1 \mathrm{H}, \mathrm{tt}, J=7.2$ and $1.3 \mathrm{~Hz}$, $\left.H 4^{\prime \prime}\right) ;{ }^{13} \mathrm{C}$ NMR (75 MHz, CD $\left.3 \mathrm{OD}\right): \delta 158.7\left(C 2^{\prime}\right), 145.4$ $(C 2(6)), 141.1\left(C 6^{\prime}\right), 140.0\left(C 3^{\prime}\right), 139.1(C 4), 138.3\left(C 1^{\prime \prime}\right), 135.0$ $\left(C_{\beta}\right), 129.5\left(C 3^{\prime \prime}\left(5^{\prime \prime}\right)\right), 129.0\left(C 4^{\prime \prime}\right), 128.3(C 3(5)), 127.9$ $\left(C 2^{\prime \prime}\left(6^{\prime \prime}\right)\right), 123.0\left(C_{\alpha}\right), 121.7\left(C 5^{\prime}\right)$. MS (CI, $\left.m / z\right): 355 / 353(39 /$ 39, M+1), 278 (41), 276 (91), 274 (61), 196 (22), 108 (26), 80 (100). Anal. Calcd for $\mathrm{C}_{17} \mathrm{H}_{13} \mathrm{BrN}_{4} \cdot 1 / 4 \mathrm{H}_{2} \mathrm{O}: \mathrm{C}, 57.08 ; \mathrm{H}$, 3.80; N, 15.66. Found: C, 57.09; H, 3.81; N, 15.46 .
5.4.6. N-[5-Bromo-3-(thiophen-3-yl)pyrazin-2-yl]pyridinium aminide (10f)

Red solid (279 mg, 84\%, ethyl acetate/diethyl ether), mp $162-165^{\circ} \mathrm{C}$; IR (KBr) $\nu_{\max }\left(\mathrm{cm}^{-1}\right): 1526,1484,1409,1369$, 1349, 1242, 1190, 1146, 1106, 807, 768; ${ }^{1} \mathrm{H}$ NMR $(300 \mathrm{MHz}$, $\left.\left(\mathrm{CD}_{3}\right)_{2} \mathrm{CO}\right): \delta 8.91\left(1 \mathrm{H}, \mathrm{dd}, J=3.1\right.$ and $\left.1.3 \mathrm{~Hz}, H 2^{\prime \prime}\right), 8.86$ $(2 \mathrm{H}, \mathrm{dd}, J=7.0$ and $1.3 \mathrm{~Hz}, H 2(6)), 8.12(1 \mathrm{H}, \mathrm{tt}, J=7.7$ and $1.3 \mathrm{~Hz}, H 4), 7.98\left(1 \mathrm{H}, \mathrm{dd}, J=5.1\right.$ and $\left.1.2 \mathrm{~Hz}, H 4^{\prime \prime}\right), 7.90(2 \mathrm{H}$, $\mathrm{dd}, J=7.7$ and $7.0 \mathrm{~Hz}, H 3(5)), 7.53\left(1 \mathrm{H}, \mathrm{s}, H 6^{\prime}\right), 7.41(1 \mathrm{H}$, $\mathrm{dd}, J=5.1$ and $\left.3.1 \mathrm{~Hz}, H 5^{\prime \prime}\right) ;{ }^{13} \mathrm{C} \mathrm{NMR}\left(75 \mathrm{MHz},\left(\mathrm{CD}_{3}\right)_{2} \mathrm{CO}\right)$ : $\delta 157.7\left(C 2^{\prime}\right), 144.4(C 2(6)), 140.3\left(C 6^{\prime}\right), 139.9\left(C 3^{\prime}\right), 137.4$ $(C 4), 136.2\left(C 3^{\prime \prime}\right), 129.2\left(C 4^{\prime \prime}\right), 127.8\left(C 2^{\prime \prime}\right), 127.6(C 3(5))$, $124.3\left(C 5^{\prime \prime}\right), 119.6\left(C 5^{\prime}\right)$. MS (CI, m/z): 335/333 (35/35, $\mathrm{M}+1), 256 / 254(67 / 64), 80$ (100). Anal. Calcd for $\mathrm{C}_{13} \mathrm{H}_{9} \mathrm{BrN}_{4} \mathrm{~S}$ : C, 46.86; H, 2.72; N, 16.81, S, 9.62. Found: C, 46.90; H, 2.84; $\mathrm{N}, 16.49 ; \mathrm{S}, 9.71$.

\subsubsection{N-[3-(Benzo[b]thiophen-3-yl)-5-bromopyrazin-2-yl]- pyridinium aminide $(\mathbf{1 0 g})$}

Orange solid (309 mg, 84\%, ethyl acetate), mp 199-202 ${ }^{\circ} \mathrm{C}$; IR $(\mathrm{KBr}) \nu_{\max }\left(\mathrm{cm}^{-1}\right): 1513,1481,1405,1215,1145,855,811$, 782, 754, 730, 660, 514, 472; ${ }^{1} \mathrm{H}$ NMR (300 MHz, $\left.\left(\mathrm{CD}_{3}\right)_{2} \mathrm{CO}\right)$ : $\delta 9.39\left(1 \mathrm{H}, \mathrm{s}, H 2^{\prime \prime}\right), 9.07\left(1 \mathrm{H}, \mathrm{m}, H 4^{\prime \prime}\right), 8.86(2 \mathrm{H}, \mathrm{dd}, J=7.0$ and $1.3 \mathrm{~Hz}, H 2(6)), 8.15(1 \mathrm{H}, \mathrm{tt}, J=7.8$ and $1.3 \mathrm{~Hz}, H 4), 7.97(1 \mathrm{H}$, $\left.\mathrm{m}, \mathrm{H}^{\prime \prime}\right), 7.92(2 \mathrm{H}, \mathrm{dd}, J=7.8$ and $7.0 \mathrm{~Hz}, H 3(5)), 7.57(1 \mathrm{H}, \mathrm{s}$, $\left.H 6^{\prime}\right), 7.46\left(1 \mathrm{H}\right.$, ddd, $J=8.0,7.0$ and $1.3 \mathrm{~Hz}, H 5^{\prime \prime}$ or $\left.H 6^{\prime \prime}\right), 7.39$ (1H, ddd, $J=7.7,7.4$ and $1.5 \mathrm{~Hz}, H 6^{\prime \prime}$ or $\left.H 5^{\prime \prime}\right) ;{ }^{13} \mathrm{C} \mathrm{NMR}$ $\left(75 \mathrm{MHz},\left(\mathrm{CD}_{3}\right)_{2} \mathrm{CO}\right): \delta 158.4\left(C 2^{\prime}\right), 144.6(C 2(6)), 140.4$ $\left(C 7 \mathrm{a}^{\prime \prime}\right), 140.1\left(C 6^{\prime}\right), 139.4\left(C 3 \mathrm{a}^{\prime \prime}\right), 137.8(C 4), 136.6\left(C 3^{\prime}\right)$, $132.0\left(\mathrm{C}^{\prime \prime}\right), 131.4\left(\mathrm{C} 2^{\prime \prime}\right), 127.7(C 3(5)), 126.8\left(\mathrm{C}^{\prime \prime}\right), 125.0$ $\left(C 5^{\prime \prime}\right.$ or $\left.C 6^{\prime \prime}\right), 125.0\left(C 6^{\prime \prime}\right.$ or $\left.C 5^{\prime \prime}\right), 123.1\left(\mathrm{C}^{\prime \prime}\right), 118.8\left(C 5^{\prime}\right)$. MS (CI, m/z): 385/383 (94/100, M+1), 306/304 (39/38), 113 (11). Anal. Calcd for $\mathrm{C}_{17} \mathrm{H}_{11} \mathrm{BrN}_{4} \mathrm{~S}$ : C, 53.28; H, 2.89; N, 14.62; S, 8.37. Found: C, 53.55; H, 2.91; N, 14.45; S, 8.17.

\subsubsection{N-[5-Bromo-3-(pyridin-3-yl)pyrazin-2-yl]pyridinium aminide (10h)}

Orange solid (204 mg, 65\%, ethyl acetate/diethyl ether), $\operatorname{mp~} 170-172{ }^{\circ} \mathrm{C}$; IR $(\mathrm{KBr}) \nu_{\max }\left(\mathrm{cm}^{-1}\right): 1524,1489,1475$, 1430, 1401, 1369, 812, 670; ${ }^{1} \mathrm{H}$ NMR (300 MHz, $\left.\mathrm{CD}_{3} \mathrm{OD}\right)$ : $\delta 9.44\left(1 \mathrm{H}, \mathrm{dd}, J=2.3\right.$ and $\left.0.8 \mathrm{~Hz}, H 2^{\prime \prime}\right), 8.74(2 \mathrm{H}, \mathrm{dd}, J=$ 6.9 and $1.3 \mathrm{~Hz}, H 2(6)), 8.73(1 \mathrm{H}, \mathrm{ddd}, J=8.1,2.3$ and $\left.1.7 \mathrm{~Hz}, H 4^{\prime \prime}\right), 8.51\left(1 \mathrm{H}, \mathrm{dd}, J=4.9\right.$ and $\left.1.7 \mathrm{~Hz}, H 6^{\prime \prime}\right), 8.15$ $(1 \mathrm{H}, \mathrm{tt}, J=7.8$ and $1.3 \mathrm{~Hz}, H 4), 7.87(2 \mathrm{H}, \mathrm{dd}, J=7.8$ and $6.9 \mathrm{~Hz}, H 3(5)), 7.63\left(1 \mathrm{H}, \mathrm{s}, H 6^{\prime}\right), 7.51(1 \mathrm{H}, \mathrm{ddd}, J=8.1,4.9$ and $\left.0.8 \mathrm{~Hz}, H 5^{\prime \prime}\right) ;{ }^{13} \mathrm{C} \mathrm{NMR}\left(75 \mathrm{MHz}, \mathrm{CD}_{3} \mathrm{OD}\right): \delta 159.1$ $\left(C 2^{\prime}\right), 150.0\left(C 2^{\prime \prime}\right), 148.6\left(C 6^{\prime \prime}\right), 145.1(C 2(6)), 142.8\left(C 6^{\prime}\right)$, 139.0 (C4), $138.0\left(C 4^{\prime \prime}\right), 137.6\left(C 3^{\prime}\right), 135.0\left(C 3^{\prime \prime}\right), 128.2$ $(C 3(5)), 124.2\left(C 5^{\prime \prime}\right), 120.9\left(C 5^{\prime}\right)$. HRMS (FAB) calcd for $\mathrm{C}_{14} \mathrm{H}_{11}^{79} \mathrm{BrN}_{5}:[\mathrm{M}+\mathrm{H}]^{+}$328.0198, found: 328.0205.

\subsubsection{N-[5-Bromo-3-(pyridin-4-yl)pyrazin-2-yl]pyridinium aminide (10i)}

Green solid (252 mg, 77\%, toluene), mp 229-231 ${ }^{\circ} \mathrm{C}$; IR $(\mathrm{KBr}) \nu_{\max }\left(\mathrm{cm}^{-1}\right): 1593,1546,1520,1484,1427,1366$, 1255, 1174, 1144, 823, 753, 658, 538; ${ }^{1} \mathrm{H}$ NMR $(300 \mathrm{MHz}$, $\left.\left(\mathrm{CD}_{3}\right)_{2} \mathrm{CO}\right): \delta 8.82(2 \mathrm{H}, \mathrm{dd}, J=7.0$ and $1.3 \mathrm{~Hz}, H 2(6)), 8.59$ 
$\left(2 \mathrm{H}, \mathrm{m}, H 2^{\prime \prime}\left(6^{\prime \prime}\right)\right), 8.44\left(2 \mathrm{H}, \mathrm{m}, H 3^{\prime \prime}\left(5^{\prime \prime}\right)\right), 8.18(1 \mathrm{H}, \mathrm{tt}, J=$ 7.7 and $1.3 \mathrm{~Hz}, H 4), 7.94(2 \mathrm{H}, \mathrm{dd}, J=7.7$ and $7.0 \mathrm{~Hz}$, $H 3(5)), 7.65\left(1 \mathrm{H}, \mathrm{s}, H 6^{\prime}\right) ;{ }^{13} \mathrm{C}$ NMR $\left(75 \mathrm{MHz},\left(\mathrm{CD}_{3}\right)_{2} \mathrm{CO}\right)$ : $\delta 159.3\left(C 2^{\prime}\right), 150.1\left(C 2^{\prime \prime}\left(6^{\prime \prime}\right), 145.5\left(C 4^{\prime \prime}\right), 144.7(C 2(6))\right.$, $143.8\left(C 6^{\prime}\right), 140.4\left(C 3^{\prime}\right), 138.3(C 4), 128.0(C 3(5)), 123.5$ $\left(C 3^{\prime \prime}\left(5^{\prime \prime}\right), 120.1\left(C 5^{\prime}\right)\right.$. MS (CI, $\left.m / z\right): 330 / 328(95 / 100, \mathrm{M}+1)$, 249 (12), 248 (22). Anal. Calcd for $\mathrm{C}_{14} \mathrm{H}_{10} \mathrm{BrN}_{5} \cdot 1 / 2 \mathrm{H}_{2} \mathrm{O}$ : C, 49.87; H, 3.29; N, 20.77. Found: C, 49.50; H, 3.54; N, 20.70.

\subsubsection{N-[5-Bromo-3-(pyrimidin-5-yl)pyrazin-2-yl]-} pyridinium aminide $(\mathbf{1 0 j})$

Orange solid (197 mg, 60\%, toluene), mp 234-236 ${ }^{\circ} \mathrm{C}$; IR $(\mathrm{KBr}) \nu_{\max }\left(\mathrm{cm}^{-1}\right): 1614,1527,1476,1463,1414,1204,1042$, 788, 749; ${ }^{1} \mathrm{H}$ NMR $\left(300 \mathrm{MHz},\left(\mathrm{CD}_{3}\right)_{2} \mathrm{CO}\right): \delta 9.74(2 \mathrm{H}, \mathrm{s}$, $\left.\mathrm{H} 4^{\prime \prime \prime}\left(6^{\prime \prime \prime}\right)\right), 9.07\left(1 \mathrm{H}, \mathrm{s}, \mathrm{H} 2^{\prime \prime \prime}\right), 8.86(2 \mathrm{H}, \mathrm{dd}, J=6.9$ and $1.3 \mathrm{~Hz}, H 2(6)), 8.19(1 \mathrm{H}, \mathrm{tt}, J=7.7$ and $1.3 \mathrm{~Hz}, H 4), 7.95$ $(2 \mathrm{H}, \mathrm{dd}, J=7.7$ and $6.9 \mathrm{~Hz}, H 3(5)), 7.65\left(1 \mathrm{H}, \mathrm{s}, H 6^{\prime}\right) ;{ }^{13} \mathrm{C}$ NMR $\left(75 \mathrm{MHz}\right.$, DMSO- $\left.d_{6}\right): \delta 157.5\left(C 2^{\prime}\right), 156.6\left(C 2^{\prime \prime}\right)$, $155.5\left(C 4^{\prime \prime}\left(6^{\prime \prime}\right)\right), 143.4(C 2(6)), 142.4\left(C 6^{\prime}\right), 137.9(C 4)$, $132.2\left(C 5^{\prime \prime}\right), 130.3\left(C 3^{\prime}\right), 127.0(C 3(5)), 118.3\left(C 5^{\prime}\right) . \mathrm{MS}$ (EI, $m / z): 330 / 328(6 / 6, \mathrm{M}+1), 202$ (31), 160 (100). Anal. Calcd for $\mathrm{C}_{13} \mathrm{H}_{9} \mathrm{BrN}_{6}$ : C, 47.44; $\mathrm{H}, 2.76 ; \mathrm{N}, 25.53$. Found: C, 47.20; H, 2.94; N, 25.80.

\subsection{Synthesis of unsymmetrical disubstituted aminides 11: general procedure}

$\operatorname{Pd}\left(\mathrm{PPh}_{3}\right)_{4}(5 \mathrm{~mol} \%)$, boronic acid $(1.5 \mathrm{mmol})$, the corresponding aminide $10(1 \mathrm{mmol})$ and $\mathrm{K}_{2} \mathrm{CO}_{3}(10 \mathrm{mmol})$ were stirred and heated under reflux under argon in a mixture of toluene/ethanol $(4: 1,15 \mathrm{~mL})$ until starting material was no longer detected by TLC (see Table 4). The system was then allowed to reach room temperature, the mixture was filtered through Celite or silica gel and the residue washed well with acetonitrile. The filtrates were combined and evaporated to dryness. Aminide $\mathbf{1 1}$ was purified by flash chromatography on a silica gel column, using ethanol as eluent, and recrystallized from the appropriate solvent where possible. Compound $\mathbf{1 1} \mathbf{j}$ was obtained as an oil. Compounds 11a, 11b, 11f and 11g were treated with aqueous hydrobromic acid (48\%) (3 equiv) and the corresponding salt was isolated after removal of excess acid. Ethyl acetate was then added and the aminide hydrobromide was triturated in an ultrasonic bath. The remaining solid was filtered off and dried. To recover the free aminides 11a, $11 \mathrm{~b}$ and $11 \mathrm{~g}$, hydrobromide was poured into acetone and ion exchange resin Amberlite IRA-67 ( 3 equiv $C_{\mathrm{E}}=1.6$ equiv/g) was added. When deprotonation was complete, the resin was removed by filtration and the solvent evaporated to give the desired aminide, which was used without further purification for other synthetic steps. Compound 11f was purified as the hydrobromide.

\subsubsection{N-[5-(3,5-Dimethylphenyl)-3-phenylpyrazin-2-yl]- pyridinium aminide (11a)}

Red solid (348 mg, 99\%), mp $72-74{ }^{\circ} \mathrm{C}$; IR (KBr) $\nu_{\max }$ $\left(\mathrm{cm}^{-1}\right): 3051,2915,1599,1500,1404,1240,1143,1028,848$, $758,695,666,542 ;{ }^{1} \mathrm{H}$ NMR $\left(300 \mathrm{MHz},\left(\mathrm{CD}_{3}\right)_{2} \mathrm{CO}\right): \delta 8.95$
$(2 \mathrm{H}, \mathrm{dd}, J=7.0$ and $1.3 \mathrm{~Hz}, H 2(6)), 8.50(2 \mathrm{H}, \mathrm{dd}, J=8.4$ and $\left.1.4 \mathrm{~Hz}, H 2^{\prime \prime}\left(6^{\prime \prime}\right)\right), 8.13\left(1 \mathrm{H}, \mathrm{s}, H 6^{\prime}\right), 8.04(1 \mathrm{H}, \mathrm{tt}, J=7.7$ and $1.3 \mathrm{~Hz}, H 4), 7.86(2 \mathrm{H}, \mathrm{dd}, J=7.7$ and $7.0 \mathrm{~Hz}, H 3(5)), 7.61$ $\left(2 \mathrm{H}\right.$, br s, $\left.H 2^{\prime \prime \prime}\left(6^{\prime \prime \prime}\right)\right), 7.38\left(3 \mathrm{H}, \mathrm{m}, H 3^{\prime \prime}, 4^{\prime \prime}\right.$ and $\left.5^{\prime \prime}\right), 6.89$ $\left(1 \mathrm{H}\right.$, br s, $\left.\mathrm{H}^{\prime \prime \prime}\right), 2.32\left(6 \mathrm{H}, \mathrm{s}, \mathrm{CH}_{3}\right) ;{ }^{13} \mathrm{C}$ NMR $(75 \mathrm{MHz}$, $\left.\left(\mathrm{CD}_{3}\right)_{2} \mathrm{CO}\right): \delta 158.1\left(C 2^{\prime}\right), 144.0(C 2(6)), 140.4\left(C 3^{\prime \prime \prime}\left(5^{\prime \prime \prime}\right)\right)$, $139.3\left(C 1^{\prime \prime}\right), 138.5\left(C 3^{\prime}\right), 138.1\left(C 1^{\prime \prime \prime}\right), 136.8\left(C 6^{\prime}\right), 136.3$ $(C 4), 132.7\left(C 5^{\prime}\right), 130.3\left(C 2^{\prime \prime \prime}\left(6^{\prime \prime \prime}\right)\right), 128.9\left(C 4^{\prime \prime}\right), 128.4$ $\left(C 3^{\prime \prime}\left(5^{\prime \prime}\right)\right), 128.0(C 3(5)), 127.5\left(C 4^{\prime \prime \prime}\right), 123.1\left(C 2^{\prime \prime \prime}\left(6^{\prime \prime \prime}\right)\right), 21.5$ $\left(\mathrm{CH}_{3}\right)$. MS (ESI, $\left.\mathrm{m} / \mathrm{z}\right): 353$ (66, M+1), 274 (100), 245 (16). HRMS (ESI-TOF, $\mathrm{CH}_{3} \mathrm{OH}$ ) calcd for $\mathrm{C}_{23} \mathrm{H}_{21} \mathrm{~N}_{4}:[\mathrm{M}+\mathrm{H}]^{+}$ 353.17607, found: 353.17960 .

\subsubsection{N-[3-(3,5-Dimethylphenyl)-5-phenylpyrazin-2-yl]- pyridinium aminide $(\mathbf{1 1 b})$}

Dark red solid (334 mg, 95\%), mp hydrobromide 173$175^{\circ} \mathrm{C}$; IR $(\mathrm{KBr}) \nu_{\max }\left(\mathrm{cm}^{-1}\right): 3106,1662,1595,1555$, $1507,1485,1465,1401,1219,855,769,760,689,667,591$; ${ }^{1} \mathrm{H}$ NMR $\left(300 \mathrm{MHz},\left(\mathrm{CD}_{3}\right)_{2} \mathrm{CO}\right): \delta 8.94(2 \mathrm{H}, \mathrm{dd}, J=7.0$ and $1.3 \mathrm{~Hz}, H 2(6)), 8.14\left(1 \mathrm{H}, \mathrm{s}, H 6^{\prime}\right), 8.07\left(2 \mathrm{H}, \mathrm{br} \mathrm{s}, H 2^{\prime \prime}\left(6^{\prime \prime}\right)\right)$, $8.05(1 \mathrm{H}, \mathrm{tt}, J=7.7$ and $1.3 \mathrm{~Hz}, H 4), 7.98(2 \mathrm{H}, \mathrm{dd}, J=8.4$ and $\left.1.3 \mathrm{~Hz}, H 2^{\prime \prime \prime}\left(6^{\prime \prime \prime}\right)\right), 7.86(2 \mathrm{H}$, dd, $J=7.7$ and $7.0 \mathrm{~Hz}$, $H 3(5)), 7.38\left(2 \mathrm{H}\right.$, ap t, $J=7.7 \mathrm{~Hz} H 3^{\prime \prime \prime}\left(5^{\prime \prime \prime}\right), 7.23(1 \mathrm{H}, \mathrm{tt}, J=$ 7.3 and $\left.1.3 \mathrm{~Hz}, H 4^{\prime \prime \prime}\right), 6.98\left(1 \mathrm{H}\right.$, br s, $\left.H 4^{\prime \prime}\right), 2.35(6 \mathrm{H}, \mathrm{s}$ $\left.\mathrm{CH}_{3}\right) ;{ }^{13} \mathrm{C}$ NMR $\left(75 \mathrm{MHz},\left(\mathrm{CD}_{3}\right)_{2} \mathrm{CO}\right): \delta 157.8\left(\mathrm{C}^{\prime}\right), 144.1$ $(C 2(6)), 140.4\left(C 3^{\prime \prime}\left(5^{\prime \prime}\right)\right), 140.1\left(C 1^{\prime \prime}\right.$ or $\left.C 1^{\prime \prime \prime}\right), 139.5\left(C 1^{\prime \prime \prime}\right.$ or $\left.C 1^{\prime \prime}\right), 137.7\left(C 3^{\prime}\right), 137.1\left(C 6^{\prime}\right), 136.6(C 4), 136.5\left(C 5^{\prime}\right)$, $130.0\left(C 4^{\prime \prime}\right), 129.3\left(C 3^{\prime \prime \prime}\left(5^{\prime \prime \prime}\right)\right), 128.2(C 3(5)), 127.5\left(C 2^{\prime \prime}\left(6^{\prime \prime}\right)\right)$, $127.2\left(C 4^{\prime \prime \prime}\right), 125.1\left(C 2^{\prime \prime \prime}\left(6^{\prime \prime \prime}\right)\right)$, $21.6\left(\mathrm{CH}_{3}\right)$. MS (ESI, $\left.\mathrm{m} / z\right)$ : 353(52, M+1), 302(10), 274(100), 245(8). HRMS (ESI-TOF, $\left.\mathrm{CH}_{3} \mathrm{OH}\right)$ calcd for $\mathrm{C}_{23} \mathrm{H}_{21} \mathrm{~N}_{4}:[\mathrm{M}+\mathrm{H}]^{+} 353.17607$, found: 353.17911 .

\subsubsection{N-[5-(Benzo[b]thiophen-3-yl)-3-phenylpyrazin-2-yl]- pyridinium aminide (11c)}

Red solid (346 mg, 89\%, ethyl acetate), mp 82-84 ${ }^{\circ} \mathrm{C}$; IR (KBr) $\nu_{\max }\left(\mathrm{cm}^{-1}\right): 3053,1667,1515,1485,1405,1141,760$, 735, 696, 665, 535; ${ }^{1} \mathrm{H}$ NMR $\left(300 \mathrm{MHz},\left(\mathrm{CD}_{3}\right)_{2} \mathrm{CO}\right): \delta 9.05$ $(2 \mathrm{H}, \mathrm{dd}, J=7.0$ and, $1.3 \mathrm{~Hz}, H 2(6)), 8.68\left(1 \mathrm{H}, \mathrm{m}, H 4^{\prime \prime \prime}\right), 8.50$ $\left(2 \mathrm{H}, \mathrm{m}, H 2^{\prime \prime}\left(6^{\prime \prime}\right)\right), 8.20(1 \mathrm{H}, \mathrm{tt}, J=7.7$ and $1.3 \mathrm{~Hz}, H 4), 8.19$ $\left(1 \mathrm{H}, \mathrm{s}, H 6^{\prime}\right), 7.97\left(3 \mathrm{H}, \mathrm{m}, H 3(5)\right.$ and $\left.7^{\prime \prime \prime}\right), 7.89\left(1 \mathrm{H}, \mathrm{s}, H 2^{\prime \prime \prime}\right)$, $7.43\left(5 \mathrm{H}, \mathrm{m}, H 3^{\prime \prime}\left(5^{\prime \prime}\right), H 4^{\prime \prime}, H 5^{\prime \prime \prime}\right.$ and $\left.H 6^{\prime \prime \prime}\right) ;{ }^{13} \mathrm{C}$ NMR $\left(75 \mathrm{MHz},\left(\mathrm{CD}_{3}\right)_{2} \mathrm{CO}\right): \delta 155.9\left(C 2^{\prime}\right), 145.0(C 2(6)), 141.6$ $\left(C 7 \mathrm{a}^{\prime \prime \prime}\right), 140.1\left(C 3 \mathrm{a}^{\prime \prime \prime}\right), 139.4\left(C 1^{\prime \prime}\right), 138.7(C 4), 138.6\left(C 6^{\prime}\right)$, $137.8\left(C 3^{\prime}\right), 137.8\left(C 5^{\prime}\right), 135.4\left(C 3^{\prime \prime \prime}\right), 130.3\left(C 2^{\prime \prime}\left(6^{\prime \prime}\right)\right), 128.8$ $\left(C 4^{\prime \prime}\right), 128.4\left(C 3^{\prime \prime}\left(5^{\prime \prime}\right)\right), 127.9(C 3(5)), 125.5\left(C 4^{\prime \prime \prime}\right), 125.2$ $\left(C 6^{\prime \prime \prime}\right), 125.1\left(C 5^{\prime \prime \prime}\right), 123.5\left(C 7^{\prime \prime \prime}\right), 123.1\left(C 2^{\prime \prime \prime}\right)$. HRMS (ESITOF, $\left.\mathrm{CH}_{3} \mathrm{OH}\right)$ calcd for $\mathrm{C}_{23} \mathrm{H}_{17} \mathrm{~N}_{4} \mathrm{~S}$ : $[\mathrm{M}+\mathrm{H}]^{+} 381.11684$, found: 381.12026 .

\subsubsection{N-[3-(Benzo[b]thiophen-3-yl)-5-phenylpyrazin-2-yl]- pyridinium aminide $(\mathbf{1 1 d})$}

Red solid (338 mg, 89\%, ethyl acetate), mp $90-92^{\circ} \mathrm{C}$; IR $(\mathrm{KBr}) \nu_{\max }\left(\mathrm{cm}^{-1}\right): 3055,1483,1448,1412,1368,1209$, $1145,1019,763,734,694,665 ;{ }^{1} \mathrm{H}$ NMR $(500 \mathrm{MHz}$, $\left.\left(\mathrm{CD}_{3}\right)_{2} \mathrm{CO}\right): \delta 9.26\left(1 \mathrm{H}, \mathrm{s}, H 2^{\prime \prime}\right), 9.20(1 \mathrm{H}, \mathrm{ddd}, J=8.2,1.3$ and $\left.0.7 \mathrm{~Hz}, H 4^{\prime \prime}\right), 8.99(2 \mathrm{H}, \mathrm{dd}, J=7.0$ and $1.3 \mathrm{~Hz}, H 2(6))$, 
$8.23\left(1 \mathrm{H}, \mathrm{s}, H 6^{\prime}\right), 8.11(1 \mathrm{H}, \mathrm{tt}, J=7.7$ and $1.3 \mathrm{~Hz}, H 4), 8.07$ $\left(2 \mathrm{H}, \mathrm{m}, H 2^{\prime \prime \prime}\left(6^{\prime \prime \prime}\right)\right), 8.03(1 \mathrm{H}$, ddd, $J=8.1,1.3$ and $0.7 \mathrm{~Hz}$, $\left.H 7^{\prime \prime}\right), 7.92(2 \mathrm{H}, \mathrm{dd}, J=7.7$ and $7.0 \mathrm{~Hz}, H 3(5)), 7.51(1 \mathrm{H}$, ddd, $J=7.6,7.0$ and $1.2 \mathrm{~Hz}, H 5^{\prime \prime}$ or $\left.H 6^{\prime \prime}\right), 7.47(2 \mathrm{H}, \mathrm{dd}, J=$ 8.2 and $7.4 \mathrm{~Hz}, H 3^{\prime \prime \prime}\left(5^{\prime \prime \prime}\right), 7.46\left(1 \mathrm{H}, \mathrm{m}, H 6^{\prime \prime}\right.$ or $\left.H 5^{\prime \prime}\right), 7.31$ $\left(1 \mathrm{H}, \mathrm{tt}, J=7.4\right.$ and $\left.1.2 \mathrm{~Hz}, H 4^{\prime \prime \prime}\right) ;{ }^{13} \mathrm{C}$ NMR $(75 \mathrm{MHz}$, $\left.\left(\mathrm{CD}_{3}\right)_{2} \mathrm{CO}\right): \delta 158.2\left(C 2^{\prime}\right), 144.4(C 2(6)), 140.4\left(C 7 \mathrm{a}^{\prime \prime}\right)$, $140.1\left(C 3 \mathrm{a}^{\prime \prime}\right), 139.7\left(C 1^{\prime \prime \prime}\right), 136.8(C 4), 136.6\left(\mathrm{C}^{\prime}\right.$ or $\left.C 5^{\prime}\right)$, $136.5\left(C 5^{\prime}\right.$ or $\left.C 3^{\prime}\right), 136.2\left(C 6^{\prime}\right), 133.7\left(C 3^{\prime \prime}\right) 130.5\left(C 2^{\prime \prime}\right)$, $129.4\left(C 3^{\prime \prime \prime}\left(5^{\prime \prime \prime}\right)\right) 127.5(C 3(5)), 127.1\left(C 4^{\prime \prime \prime}\right.$ or $\left.C 4^{\prime \prime}\right), 126.9$ $\left(C 4^{\prime \prime}\right.$ or $\left.C 4^{\prime \prime \prime}\right), 125.1\left(C 2^{\prime \prime \prime}\left(6^{\prime \prime \prime}\right)\right), 125.0\left(C 5^{\prime \prime}\right.$ or $\left.C 6^{\prime \prime}\right), 124.9$, $\left(C 6^{\prime \prime}\right.$ or $\left.C 5^{\prime \prime}\right), 123.1\left(C 7^{\prime \prime}\right)$. MS (ESI, $\left.m / z\right): 381(59, \mathrm{M}+1), 303$ (30), 302 (100). Anal. Calcd for $\mathrm{C}_{23} \mathrm{H}_{16} \mathrm{~N}_{4} \mathrm{~S}: \mathrm{C}, 72.61 ; \mathrm{H}, 4.24$; N, 14.73; S, 8.43. Found: C, 72.29; H, 4.41; N, 14.36; S, 8.08.

\subsubsection{N-[3-Phenyl-5-(pyridin-3-yl)pyrazin-2-yl]pyridinium aminide (11e)}

See Ref. 10b.

\subsubsection{N-[5-Phenyl-3-(pyridin-3-yl)pyrazin-2-yl]pyridinium aminide (11f)}

Orange solid (221 mg, 68\%), mp hydrobromide 207$209^{\circ} \mathrm{C}$ (yellowish solid, ethanol); IR $(\mathrm{KBr}) \nu_{\max }\left(\mathrm{cm}^{-1}\right)$ : 1507, 1488, 1401, 1370, 1144, 757, 696, 665; ${ }^{1} \mathrm{H}$ NMR $\left(300 \mathrm{MHz}, \mathrm{CD}_{3} \mathrm{OD}\right): \delta 9.52\left(1 \mathrm{H}, \mathrm{d}, J=2.2 \mathrm{~Hz}, H 2^{\prime \prime}\right), 8.82$ $\left(1 \mathrm{H}, \mathrm{dt}, J=8.1\right.$ and $\left.1.9 \mathrm{~Hz}, H 6^{\prime \prime}\right), 8.77(2 \mathrm{H}, \mathrm{dd}, J=6.7$ and $1.3 \mathrm{~Hz}, H 2(6)), 8.52\left(1 \mathrm{H}, \mathrm{dd}, J=5.0\right.$ and $\left.1.6 \mathrm{~Hz}, H 4^{\prime \prime}\right), 8.11$ $(1 \mathrm{H}, \mathrm{tt}, J=7.6$ and $1.3 \mathrm{~Hz}, H 4), 8.09\left(1 \mathrm{H}, \mathrm{s}, H 6^{\prime}\right), 7.91(2 \mathrm{H}$, ap dd, $J=7.3$ and $\left.1.4 \mathrm{~Hz}, H 2^{\prime \prime \prime}\left(6^{\prime \prime \prime}\right)\right), 7.85(2 \mathrm{H}$, dd, $J=7.6$ and $6.7 \mathrm{~Hz}, H 3(5)), 7.52\left(1 \mathrm{H}, \mathrm{dd}, J=8.1\right.$ and $\left.5.0 \mathrm{~Hz}, H 5^{\prime \prime}\right), 7.40$ $\left(2 \mathrm{H}\right.$, ap t $\left., J=7.3 \mathrm{~Hz}, H 3^{\prime \prime \prime}\left(5^{\prime \prime \prime}\right)\right), 7.28(1 \mathrm{H}, \mathrm{tt}, J=7.3$ and $\left.1.4 \mathrm{~Hz}, H 4^{\prime \prime \prime}\right) ;{ }^{13} \mathrm{C}$ NMR $\left(75 \mathrm{MHz}, \mathrm{CD}_{3} \mathrm{OD}\right): \delta 159.0\left(C 2^{\prime}\right)$, $150.5\left(C 2^{\prime \prime}\right), 148.5\left(C 4^{\prime \prime}\right), 145.2(C 2(6)), 138.9\left(C 1^{\prime \prime \prime}\right), 138.7$ $(C 4), 138.5\left(C 6^{\prime \prime}\right), 138.2\left(C 5^{\prime}\right), 138.0\left(C 6^{\prime}\right), 137.3\left(C 3^{\prime}\right), 136.5$ $\left(C 3^{\prime \prime}\right), 129.5\left(C 3^{\prime \prime \prime}\left(5^{\prime \prime \prime}\right)\right), 128.3(C 3(5)), 127.8\left(C 4^{\prime \prime \prime}\right), 125.5$ $\left(C 2^{\prime \prime \prime}\left(6^{\prime \prime \prime}\right)\right), 124.3\left(C 5^{\prime \prime}\right)$. MS (CI, $\left.m / z\right): 326(100, \mathrm{M}+1), 277$ (18), 249 (83), 80 (39). Anal. Calcd for $\mathrm{C}_{20} \mathrm{H}_{17} \mathrm{BrN}_{5} \cdot 1 / 2 \mathrm{H}_{2} \mathrm{O}$ : C, 48.41; H, 3.66; N, 14.11. Found: C, 48.45; H, 3.76; N, 13.82 .

\subsection{7. $N$-[5-(Benzo[b]thiophen-3-yl)-3-(3,5-dimethyl-}

phenyl)pyrazin-2-yl]pyridinium aminide (11g)

Orange solid (367 mg, 90\%), mp 69-71 ${ }^{\circ} \mathrm{C}$; IR (KBr) $\nu_{\max }$ $\left(\mathrm{cm}^{-1}\right): 2917,1600,1515,1470,1397,1210,1140,761,735$, 669; ${ }^{1} \mathrm{H}$ NMR $\left(300 \mathrm{MHz},\left(\mathrm{CD}_{3}\right)_{2} \mathrm{CO}\right): \delta 8.95(2 \mathrm{H}, \mathrm{dd}, J=6.8$ and $1.2 \mathrm{~Hz}, H 2(6)), 8.68\left(1 \mathrm{H}, \mathrm{m}, H 4^{\prime \prime \prime}\right), 8.12(2 \mathrm{H}, \mathrm{br}$ s, $\left.H 2^{\prime \prime}\left(6^{\prime \prime}\right)\right), 8.09\left(1 \mathrm{H}, \mathrm{s}, H 6^{\prime}\right), 8.05(1 \mathrm{H}, \mathrm{tt}, J=6.8$ and $1.2 \mathrm{~Hz}$, $H 4), 7.96\left(1 \mathrm{H}, \mathrm{m}, H 7^{\prime \prime \prime}\right), 7.87(2 \mathrm{H}, \mathrm{dd}, J=7.6$ and $6.8 \mathrm{~Hz}$, $H 3(5)), 7.80\left(1 \mathrm{H}, \mathrm{s}, H 2^{\prime \prime \prime}\right), 7.41\left(2 \mathrm{H}, \mathrm{m}, H 5^{\prime \prime \prime}\right.$ and $\left.6^{\prime \prime \prime}\right), 7.00$ $\left(1 \mathrm{H}\right.$, br s, $\left.\mathrm{H}^{\prime \prime}\right), 2.35\left(6 \mathrm{H}, \mathrm{s}, \mathrm{CH}_{3}\right) ;{ }^{13} \mathrm{C}$ NMR $(75 \mathrm{MHz}$, $\left.\left(\mathrm{CD}_{3}\right)_{2} \mathrm{CO}\right): \delta$ 157.6(C2'), $144.2(C 2(6)), 141.6\left(C 7 \mathrm{a}^{\prime \prime \prime}\right)$, $140.1\left(C 3^{\prime \prime}\left(5^{\prime \prime}\right)\right), 140.0\left(C 3 \mathrm{a}^{\prime \prime \prime}\right), 138.8\left(C 1^{\prime \prime}\right), 138.6(C 4), 137.1$ $\left(C 6^{\prime}\right), 136.4\left(C 3^{\prime}\right), 136.2\left(C 5^{\prime}\right), 136.1\left(C 3^{\prime \prime \prime}\right), 130.0\left(C 4^{\prime \prime}\right)$, $128.1(C 3(5)), 127.5\left(C 2^{\prime \prime}\left(6^{\prime \prime}\right)\right), 125.6\left(C 4^{\prime \prime \prime}\right), 125.1\left(C 5^{\prime \prime \prime}\right.$ or $\left.C 6^{\prime \prime \prime}\right), 124.9\left(C 6^{\prime \prime \prime}\right.$ or $\left.C 5^{\prime \prime \prime}\right), 123.4\left(C 7^{\prime \prime \prime}\right), 121.9\left(C 2^{\prime \prime \prime}\right), 21.6$ $\left(\mathrm{CH}_{3}\right)$. HRMS (ESI-TOF, $\mathrm{CH}_{3} \mathrm{OH}$ ) calcd for $\mathrm{C}_{25} \mathrm{H}_{21} \mathrm{~N}_{4} \mathrm{~S}$ : $[\mathrm{M}+\mathrm{H}]^{+}$409.14814, found: 409.15105 .
5.5.8. N-[3-(Benzo[b]thiophen-3-yl)-5-(3,5-dimethyl-

phenyl)pyrazin-2-yl]pyridinium aminide (11h)

Orange solid ( $335 \mathrm{mg}, 82 \%$, ethyl acetate), $\mathrm{mp} 77-79{ }^{\circ} \mathrm{C}$; IR (KBr) $\nu_{\max }\left(\mathrm{cm}^{-1}\right): 2913,1600,1492,1406,1360,1205$, 1145, 760, 733, 697, 667; ${ }^{1} \mathrm{H}$ NMR $\left(300 \mathrm{MHz},\left(\mathrm{CD}_{3}\right)_{2} \mathrm{CO}\right)$ : $\delta 9.19\left(1 \mathrm{H}, \mathrm{s}, H 2^{\prime \prime}\right), 9.16\left(1 \mathrm{H}, \mathrm{m}, H 4^{\prime \prime}\right), 8.96(2 \mathrm{H}, \mathrm{dd}, J=7.0$ and $1.3 \mathrm{~Hz}, H 2(6)), 8.16\left(1 \mathrm{H}, \mathrm{s}, H 6^{\prime}\right), 8.06(1 \mathrm{H}, \mathrm{tt}, J=7.7$ and $1.3 \mathrm{~Hz}, H 4), 7.98\left(1 \mathrm{H}, \mathrm{m}, H 7^{\prime \prime}\right), 7.88(2 \mathrm{H}, \mathrm{dd}, J=7.7$ and $7.0 \mathrm{~Hz}, H 3(5)), 7.64\left(2 \mathrm{H}\right.$, br s, $\left.H 2^{\prime \prime \prime}\left(6^{\prime \prime \prime}\right)\right), 7.46(1 \mathrm{H}, \mathrm{ddd}$, $J=7.6,7.0$ and $1.4 \mathrm{~Hz}, H 5^{\prime \prime}$ or $\left.H 6^{\prime \prime}\right), 7.39(1 \mathrm{H}$, ddd, $J=8.2$, 7.0 and $1.4 \mathrm{~Hz}, H 6^{\prime \prime}$ or $\left.H 5^{\prime \prime}\right), 6.91\left(1 \mathrm{H}\right.$, br s, $\left.H 4^{\prime \prime \prime}\right), 2.34$ $\left(6 \mathrm{H}, \mathrm{s}, \mathrm{CH}_{3}\right) ;{ }^{13} \mathrm{C}$ NMR $\left(75 \mathrm{MHz},\left(\mathrm{CD}_{3}\right)_{2} \mathrm{CO}\right): \delta 157.7\left(\mathrm{C2}^{\prime}\right)$, $144.3(C 2(6)), 140.5\left(C 3^{\prime \prime \prime}\left(5^{\prime \prime \prime}\right)\right), 140.2\left(C 7 \mathrm{a}^{\prime \prime}\right), 139.6\left(C 3 \mathrm{a}^{\prime \prime}\right)$, $138.6(C 4), 137.3\left(C 1^{\prime \prime \prime}\right), 136.8\left(C 3^{\prime}\right), 136.7\left(C 5^{\prime}\right), 136.1\left(C 6^{\prime}\right)$, $133.8\left(\mathrm{C}^{\prime \prime}\right), 130.4\left(\mathrm{C} 2^{\prime \prime}\right), 128.9\left(C 4^{\prime \prime \prime}\right), 127.5(C 3(5)), 127.0$ $\left(C 4^{\prime \prime}\right), 124.9\left(C 5^{\prime \prime}\right.$ or $\left.C 6^{\prime \prime}\right), 124.8\left(C 6^{\prime \prime}\right.$ or $\left.5^{\prime \prime}\right), 123.3$ $\left(C 2^{\prime \prime \prime}\left(6^{\prime \prime \prime}\right)\right), 123.2\left(C 7^{\prime \prime}\right), 21.6\left(\mathrm{CH}_{3}\right)$. MS (ESI, $\left.\mathrm{m} / \mathrm{z}\right): 409$ (73, M+1), 331 (25), 330 (100), 277 (9), 245 (13). HRMS (ESI-TOF, $\mathrm{CH}_{3} \mathrm{OH}$ ) calcd for $\mathrm{C}_{25} \mathrm{H}_{21} \mathrm{~N}_{4} \mathrm{~S}: \quad[\mathrm{M}+\mathrm{H}]^{+}$ 409.14814, found: 409.15118 .

\subsubsection{N-[5-(4-Methoxyphenyl)-3-(thiophen-3-yl)pyrazin-2-yl]- pyridinium aminide (11i)}

Red solid (292 mg, 81\%, ether), mp $149-150{ }^{\circ} \mathrm{C}$; IR (KBr) $\nu_{\max }\left(\mathrm{cm}^{-1}\right): 3108,2835,1607,1555,1489,1421,1368,1244$, 1182, 1144, 1033, 834, 667; ${ }^{1} \mathrm{H}$ NMR $\left(300 \mathrm{MHz},\left(\mathrm{CD}_{3}\right)_{2} \mathrm{CO}\right)$ : $\delta 8.99(2 \mathrm{H}, \mathrm{dd}, J=7.0$ and $1.2 \mathrm{~Hz}, H 2(6)), 8.95(1 \mathrm{H}, \mathrm{dd}, J=3.1$ and $\left.1.2 \mathrm{~Hz}, H 2^{\prime \prime}\right), 8.26\left(1 \mathrm{H}, \mathrm{dd}, J=5.1\right.$ and $\left.1.2 \mathrm{~Hz}, H 4^{\prime \prime}\right), 8.08$ $\left(1 \mathrm{H}, \mathrm{s}, H 6^{\prime}\right), 8.00(1 \mathrm{H}, \mathrm{tt}, J=7.6$ and $1.2 \mathrm{~Hz}, H 4), 7.93(2 \mathrm{H}$, ap $\left.\mathrm{d}, J=8.9 \mathrm{~Hz}, H 2^{\prime \prime \prime}\left(6^{\prime \prime \prime}\right)\right), 7.84(2 \mathrm{H}$, dd, $J=7.7$ and $7.0 \mathrm{~Hz}$, $H 3(5)), 7.41\left(1 \mathrm{H}, \mathrm{dd}, J=5.1\right.$ and $\left.3.1 \mathrm{~Hz}, H 5^{\prime \prime}\right), 6.97(2 \mathrm{H}$, ap $\left.\mathrm{d}, J=8.9 \mathrm{~Hz}, \mathrm{H3}^{\prime \prime \prime}\left(5^{\prime \prime \prime}\right)\right), \quad 3.80\left(3 \mathrm{H}, \mathrm{s}, \mathrm{OCH}_{3}\right) ;{ }^{13} \mathrm{C} \mathrm{NMR}$ $\left(75 \mathrm{MHz},\left(\mathrm{CD}_{3}\right)_{2} \mathrm{CO}\right): \delta 159.7\left(C 4^{\prime \prime \prime}\right), 157.0\left(C 2^{\prime}\right), 143.8$ (C2(6)), $141.6\left(\mathrm{C}^{\prime}\right), 137.0\left(C 5^{\prime}\right), 136.7\left(C 3^{\prime \prime}\right), 135.6\left(C 6^{\prime}\right.$ or $C 4), 135.3\left(C 4\right.$ or $\left.C 6^{\prime}\right), 132.3\left(C 1^{\prime \prime \prime}\right), 129.8\left(C 4^{\prime \prime}\right), 127.3$ $(C 3(5)), 127.2\left(C 2^{\prime \prime}\right), 126.4\left(C 2^{\prime \prime \prime}\left(6^{\prime \prime \prime}\right)\right), 123.8\left(C 5^{\prime \prime}\right), 114.7$, $\left(C 3^{\prime \prime \prime}\left(C 5^{\prime \prime \prime}\right)\right)$, $55.5\left(\mathrm{CH}_{3} \mathrm{O}\right)$. MS (CI, $\left.\mathrm{m} / \mathrm{z}\right): 361(100, \mathrm{M}+1)$, 360 (66), 284 (71), 282 (51), 80 (43). Anal. Calcd for $\mathrm{C}_{20} \mathrm{H}_{16} \mathrm{~N}_{4} \mathrm{OS} \cdot 1 / 2 \mathrm{H}_{2} \mathrm{O}: \mathrm{C}, 65.02 ; \mathrm{H}, 4.64 ; \mathrm{N}, 15.26 ; \mathrm{S}, 8.68$. Found: C, 64.66; H, 4.40; N, 14.96; S, 8.79.

\subsubsection{N-[3-(Benzo[b]thiophen-3-yl)-5-(trans-2-}

phenylvinyl)pyrazin-2-yllpyridinium aminide $(\mathbf{1 1 j})$

Red oil (264 mg, 65\%); IR (KBr) $\nu_{\max }\left(\mathrm{cm}^{-1}\right): 3111,3055$, 2923, 1548, 1485, 1421, 1379, 1227, 1200, 1145, 751, 692, 668; ${ }^{1} \mathrm{H}$ NMR $\left(300 \mathrm{MHz},\left(\mathrm{CD}_{3}\right)_{2} \mathrm{CO}\right): \delta 9.19\left(1 \mathrm{H}, \mathrm{s}, H 2^{\prime \prime}\right)$, $9.18\left(1 \mathrm{H}\right.$, br d, $\left.J=8.5 \mathrm{~Hz}, H 4^{\prime \prime}\right), 8.93(2 \mathrm{H}, \mathrm{dd}, J=7.0$ and $1.3 \mathrm{~Hz}, H 2(6)), 8.08(1 \mathrm{H}, \mathrm{tt}, J=7.6$ and $1.3 \mathrm{~Hz}, H 4), 7.98$ $\left(1 \mathrm{H}\right.$, br d, $\left.J=7.9 \mathrm{~Hz}, H 7^{\prime \prime}\right), 7.88(2 \mathrm{H}$, dd, $J=7.6$ and $7.0 \mathrm{~Hz}$, $H 3(5)), 7.70\left(1 \mathrm{H}, \mathrm{s}, H 6^{\prime}\right), 7.57\left(2 \mathrm{H}, \mathrm{m}, H 2^{\prime \prime \prime}\left(6^{\prime \prime \prime}\right)\right), 7.51(1 \mathrm{H}$, ddd, $J=8.2,7.0$ and $\left.1.3 \mathrm{~Hz}, H 5^{\prime \prime}\right), 7.42(1 \mathrm{H}, \mathrm{d}, J=16.0 \mathrm{~Hz}$, $\left.H_{\alpha}\right), 7.40\left(1 \mathrm{H}, \mathrm{m}, H 6^{\prime \prime}\right), 7.34\left(2 \mathrm{H}\right.$, br t, $\left.J=7.5 \mathrm{~Hz}, H 3^{\prime \prime \prime}\left(5^{\prime \prime \prime}\right)\right)$, $7.23\left(1 \mathrm{H}, \mathrm{d}, J=16.0 \mathrm{~Hz}, H_{\beta}\right), 7.19(1 \mathrm{H}, \mathrm{tt}, J=7.5$ and $\left.1.3 \mathrm{~Hz}, H 4^{\prime \prime \prime}\right) ;{ }^{13} \mathrm{C}$ NMR $\left(75 \mathrm{MHz},\left(\mathrm{CD}_{3}\right)_{2} \mathrm{CO}\right): \delta 158.3$ $\left(C 2^{\prime}\right), 144.3(C 2(6)), 140.4\left(C 7 \mathrm{a}^{\prime \prime}\right), 140.2\left(C 3 \mathrm{a}^{\prime \prime}\right), 139.5\left(\mathrm{C6}{ }^{\prime}\right)$, $139.2\left(C 1^{\prime \prime \prime}\right), 137.7\left(C 3^{\prime}\right), 136.8(C 4), 135.8\left(C 5^{\prime}\right), 133.7$ $\left(C 3^{\prime \prime}\right), 130.7\left(C 2^{\prime \prime}\right), 129.4\left(C 3^{\prime \prime \prime}\left(5^{\prime \prime \prime}\right)\right), 127.5(C 3(5)), 127.4$ 
$\left(C 4^{\prime \prime \prime}\right), 127.1\left(C 4^{\prime \prime}\right), 127.0,\left(C_{\beta}\right), 126.9\left(C 2^{\prime \prime \prime}\left(6^{\prime \prime \prime}\right)\right), 125.5,\left(C_{\alpha}\right)$, $124.9\left(C 5^{\prime \prime}\right.$ and $\left.C 6^{\prime \prime}\right), 123.1\left(C 7^{\prime \prime}\right)$. MS (ESI, $\left.m / z\right)$ : 407 (81, M+1), 329 (27), 328 (100), 277 (9), 245 (13), 133 (16). HRMS (ESI-TOF, $\mathrm{CH}_{3} \mathrm{OH}$ ) calcd for $\mathrm{C}_{25} \mathrm{H}_{19} \mathrm{~N}_{4} \mathrm{~S}$ : $[\mathrm{M}+\mathrm{H}]^{+}$ 407.13249, found: 407.13536 .

\subsubsection{N-[3-(Benzo[b]thiophen-3-yl)-5-(pyrimidin-5-yl)- pyrazin-2-yl]pyridinium aminide (11k)}

Orange solid ( $344 \mathrm{mg}, 90 \%$, ethanol), mp $215-217^{\circ} \mathrm{C}$; IR $(\mathrm{KBr}) \nu_{\max }\left(\mathrm{cm}^{-1}\right): 3111,3046,1548,1489,1405,1221$, 1146, 760, 734; ${ }^{1} \mathrm{H}$ NMR $\left(300 \mathrm{MHz},\left(\mathrm{CD}_{3}\right)_{2} \mathrm{CO}\right): \delta 9.30(2 \mathrm{H}$, s, $\left.H 4^{\prime \prime \prime}\left(6^{\prime \prime \prime}\right)\right), 9.24\left(1 \mathrm{H}, \mathrm{s}, H 2^{\prime \prime}\right), 9.05(1 \mathrm{H}$, br d, $J=7.7 \mathrm{~Hz}$, $\left.H 4^{\prime \prime}\right), 9.00\left(1 \mathrm{H}, \mathrm{s}, H 2^{\prime \prime \prime}\right), 8.91(2 \mathrm{H}$, dd, $J=7.0$ and $1.3 \mathrm{~Hz}$, $H 2(6)), 8.27\left(1 \mathrm{H}, \mathrm{s}, H 6^{\prime}\right), 8.20(1 \mathrm{H}, \mathrm{tt}, J=7.7$ and $7.0 \mathrm{~Hz}, H 4)$, $7.98\left(3 \mathrm{H}, \mathrm{m}, H 3(5)\right.$ and $\left.H 7^{\prime \prime}\right), 7.50(1 \mathrm{H}$, ddd, $J=8.2,7.0$ and $\left.1.3 \mathrm{~Hz}, H 5^{\prime \prime}\right), 7.42\left(1 \mathrm{H}, \mathrm{ddd}, J=8.1,6.8\right.$ and $\left.1.4 \mathrm{~Hz}, H 6^{\prime \prime}\right) ;{ }^{13} \mathrm{C}$ NMR $\left(75 \mathrm{MHz},\left(\mathrm{CD}_{3}\right)_{2} \mathrm{CO}\right): \delta 159.2\left(C 2^{\prime}\right), 156.9\left(C 2^{\prime \prime \prime}\right)$, $152.9\left(C 4^{\prime \prime \prime}\left(6^{\prime \prime \prime}\right)\right), 144.8(C 2(6)), 140.5\left(C 7 \mathrm{a}^{\prime \prime}\right), 139.9\left(C 3 \mathrm{a}^{\prime \prime}\right)$, $138.1(C 4), 137.4\left(C 6^{\prime}\right), 133.3\left(C 5^{\prime}\right), 132.9\left(C 3^{\prime}\right), 131.1\left(C 2^{\prime \prime}\right)$, $130.3\left(C 3^{\prime \prime}\right), 129.5\left(C 5^{\prime \prime \prime}\right), 127.8(C 3(5)), 126.6\left(C 4^{\prime \prime}\right), 125.1$ $\left(C 5^{\prime \prime}\right.$ or $\left.C 6^{\prime \prime}\right), 125.0\left(C 6^{\prime \prime}\right.$ or $\left.C 5^{\prime \prime}\right), 123.3\left(C 7^{\prime \prime}\right)$. MS (EI, $\left.m / z\right)$ : $382\left(24, \mathrm{M}^{+}\right), 303$ (100), 275 (26), 171 (24), 79(50). HRMS (ESI-TOF, $\left.\mathrm{CH}_{3} \mathrm{OH}\right)$ calcd for $\mathrm{C}_{21} \mathrm{H}_{15} \mathrm{~N}_{6} \mathrm{~S}: \quad[\mathrm{M}+\mathrm{H}]^{+}$ 383.10734, found: 383.11140. Anal. Calcd for $\mathrm{C}_{21} \mathrm{H}_{14} \mathrm{~N}_{6} \mathrm{~S}: \mathrm{C}$, 65.95; H, 3.69; N, 21.97; S, 8.38. Found: C, 65.75; H, 3.68; N, 21.70; S, 8.34.

\subsubsection{N-[5-(Benzo[b]thiophen-3-yl)-3-(pyrimidin-5-yl)- pyrazin-2-yl]pyridinium aminide (11l)}

Orange solid (328 mg, 86\%, ethanol/ethyl acetate), mp 217-220 ${ }^{\circ} \mathrm{C}$; IR (KBr) $\nu_{\max }\left(\mathrm{cm}^{-1}\right): 3051,1548,1516$, $1491,1405,1145,759 ;{ }^{1} \mathrm{H}$ NMR $\left(500 \mathrm{MHz}\right.$, DMSO- $\left.d_{6}\right)$ : $\delta 9.78\left(2 \mathrm{H}, \mathrm{s}, H 4^{\prime \prime}\left(6^{\prime \prime}\right)\right), 9.13\left(1 \mathrm{H}, \mathrm{s}, H 2^{\prime \prime}\right), 8.87(2 \mathrm{H}, \mathrm{dd}, J=$ 7.0 and $1.2 \mathrm{~Hz}, H 2(6)), 8.50\left(1 \mathrm{H}, \mathrm{d}, J=8.2 \mathrm{~Hz}, H 4^{\prime \prime \prime}\right), 8.14$ $\left(1 \mathrm{H}, \mathrm{s}, H 6^{\prime}\right), 8.13(1 \mathrm{H}, \mathrm{tt}, J=7.7$ and $1.2 \mathrm{~Hz}, H 4), 8.00(1 \mathrm{H}$, d, $\left.J=7.9 \mathrm{~Hz}, H 7^{\prime \prime \prime}\right), 7.92\left(1 \mathrm{H}, \mathrm{s}, H 2^{\prime \prime \prime}\right), 7.89(2 \mathrm{H}, \mathrm{dd}, J=7.7$ and $7.0 \mathrm{~Hz}, H 3(5)), 7.44(1 \mathrm{H}$, ddd, $J=8.0,7.1$ and $1.2 \mathrm{~Hz}$, $\left.H 5^{\prime \prime \prime}\right), 7.40\left(1 \mathrm{H}\right.$, ddd, $J=8.2,6.9$ and $\left.1.3 \mathrm{~Hz}, H 66^{\prime \prime \prime}\right) ;{ }^{13} \mathrm{C}$ NMR $\left(75 \mathrm{MHz}\right.$, DMSO- $\left.d_{6}\right): \delta 157.3\left(C 2^{\prime}\right), 156.5\left(C 2^{\prime \prime}\right), 156.0$ $\left(C 4^{\prime \prime}\left(6^{\prime \prime}\right)\right), 143.6(C 2(6)), 140.2\left(C 6^{\prime}\right), 139.8\left(C 7 \mathrm{a}^{\prime \prime \prime}\right), 137.6(C 4)$, $136.8\left(C 3 \mathrm{a}^{\prime \prime \prime}\right), 134.0\left(C 3^{\prime \prime \prime}\right), 133.5\left(C 5^{\prime}\right), 131.9\left(C 3^{\prime}\right), 130.8$ $\left(C 5^{\prime \prime}\right), 127.0(C 3(5)), 124.2\left(C 5^{\prime \prime \prime}\right.$ or $\left.C 6^{\prime \prime \prime}\right), 124.1\left(C 6^{\prime \prime \prime}\right.$ or $\left.C 5^{\prime \prime \prime}\right), 123.7\left(C 4^{\prime \prime \prime}\right), 122.6\left(C 7^{\prime \prime \prime}\right), 121.8\left(C 2^{\prime \prime \prime}\right)$. MS (EI, $\left.\mathrm{m} / \mathrm{z}\right)$ : $382\left(100, \mathrm{M}^{+}\right), 303$ (47), 275 (31), 172 (55), 79(50). Anal. Calcd for $\mathrm{C}_{21} \mathrm{H}_{14} \mathrm{~N}_{6} \mathrm{~S} \cdot 1 / 2 \mathrm{H}_{2} \mathrm{O}: \mathrm{C}, 64.43 ; \mathrm{H}, 3.86 ; \mathrm{N}, 21.47 ; \mathrm{S}$, 8.19. Found: C, 64.10; H, 3.61; N, 21.07; S, 8.07.

\subsubsection{N-[3-(Pyrimidin-5-yl)-5-(thiophen-3-yl)pyrazin-2-yl]- pyridinium aminide $(\mathbf{1 1 m})$}

Red solid (299 mg, 90\%, ethyl acetate), mp $183-185^{\circ} \mathrm{C}$; IR $(\mathrm{KBr}) \nu_{\max }\left(\mathrm{cm}^{-1}\right): 3105,2923,1546,1531,1497,1451,1397$, 1336, 1253, 1162, 1143, 1017, 758, 662; ${ }^{1} \mathrm{H}$ NMR (300 MHz, $\left.\left(\mathrm{CD}_{3}\right)_{2} \mathrm{CO}\right): \delta 9.87\left(2 \mathrm{H}, \mathrm{s}, H 4^{\prime \prime}\left(6^{\prime \prime}\right)\right), 9.07\left(1 \mathrm{H}, \mathrm{s}, H 2^{\prime \prime}\right), 8.96$ $(2 \mathrm{H}, \mathrm{dd}, J=7.0$ and $1.3 \mathrm{~Hz}, H 2(6)), 8.16\left(1 \mathrm{H}, \mathrm{s}, H 6^{\prime}\right)$, $8.10(1 \mathrm{H}, \mathrm{tt}, J=7.7$ and $1.3 \mathrm{~Hz}, H 4), 7.90(2 \mathrm{H}, \mathrm{dd}, J=$ 7.7 and $7.0 \mathrm{~Hz}, H 3(5)), 7.82\left(1 \mathrm{H}, \mathrm{dd}, J=2.9\right.$ and $\left.1.3 \mathrm{~Hz}, H 2^{\prime \prime \prime}\right)$, $7.68\left(1 \mathrm{H}, \mathrm{dd}, J=5.1\right.$ and $\left.1.3 \mathrm{~Hz}, H 4^{\prime \prime \prime}\right), 7.50(1 \mathrm{H}, \mathrm{dd}, J=5.1$ and $\left.2.9 \mathrm{~Hz}, H 5^{\prime \prime \prime}\right) ;{ }^{13} \mathrm{C}$ NMR $\left(75 \mathrm{MHz},\left(\mathrm{CD}_{3}\right)_{2} \mathrm{CO}\right): \delta 158.3$ $\left(C 2^{\prime}\right), 157.7\left(C 2^{\prime \prime}\right), 157.6\left(C 4^{\prime \prime}\left(6^{\prime \prime}\right)\right), 144.2(C 2(6)), 141.7$ (C3'), $138.9\left(C 6^{\prime}\right), 137.0(C 4), 135.6\left(C 5^{\prime}\right), 133.3\left(C 3^{\prime \prime \prime}\right), 127.9$ $\left(C 5^{\prime \prime}\right), 127.6(C 3(5)), 126.7\left(C 4^{\prime \prime \prime}\right), 126.0\left(C 2^{\prime \prime \prime}\right), 119.0\left(C 5^{\prime \prime \prime}\right)$. MS (CI, $m / z): 333$ (100, M+1), 254 (51), 80 (9). HRMS (ESITOF, $\mathrm{CH}_{3} \mathrm{OH}$ ) calcd for $\mathrm{C}_{17} \mathrm{H}_{13} \mathrm{~N}_{6} \mathrm{~S}$ : $[\mathrm{M}+\mathrm{H}]^{+} 333.09169$, found: 333.09588 .

\subsection{Reaction of 3,5-disubstituted N-(pyrazin-2'-yl)- pyridinium aminides (11) with benzyl bromides}

General method. In a dried round-bottomed flask, a solution of the corresponding aminide $(0.5 \mathrm{mmol})$ in anhydrous acetone $(5 \mathrm{~mL})$ was prepared. After addition of the corresponding alkyl bromide ( $4 \mathrm{mmol})$, the mixture was stirred at room temperature for the time indicated in Table 5, until starting aminide was no longer detected by TLC. Once the reaction was complete, the solvent was evaporated in vacuo and the remaining crude product was triturated with ethyl acetate in an ultrasonic bath. The resulting suspension was filtered and the solid washed well with ethyl acetate to remove the excess alkyl bromide. The isolated salts $\mathbf{1 2}$ were used without further purification in the next step.

\subsubsection{1 -\{N-Benzyl-N-[5-(3,5-dimethyl)phenyl-3-} phenylpyrazin-2-yl]amino\} pyridinium bromide (12a)

White solid (182 mg, 78\%, ethanol), mp $182-184{ }^{\circ} \mathrm{C}$; IR (KBr) $\nu_{\max }\left(\mathrm{cm}^{-1}\right): 3103,3033,1616,1467,1416,1371$, $1335,1240,1158,1017,861,720,700,682,481 ;{ }^{1} \mathrm{H}$ NMR (300 MHz, $\left.\mathrm{CD}_{3} \mathrm{OD}\right): \delta 8.96\left(1 \mathrm{H}, \mathrm{s}, H 6^{\prime}\right), 8.92(2 \mathrm{H}, \mathrm{dd}, J=6.8$ and $1.3 \mathrm{~Hz}, H 2(6)), 8.51(1 \mathrm{H}, \mathrm{tt}, J=7.8$ and $1.3 \mathrm{~Hz}, H 4), 7.92$ $(2 \mathrm{H}, \mathrm{dd}, J=7.8$ and $6.8 \mathrm{~Hz}, H 3(5)), 7.79\left(4 \mathrm{H}, \mathrm{m}, H 2^{\prime \prime}\left(6^{\prime \prime}\right)\right.$ and $\left.H 2^{\prime \prime \prime}\left(6^{\prime \prime \prime}\right)\right), 7.54\left(3 \mathrm{H}, \mathrm{m}, H 3^{\prime \prime}\left(5^{\prime \prime}\right)\right.$ and $\left.H 4^{\prime \prime}\right), 7.30(5 \mathrm{H}$, ap s, $\left.H-\mathrm{Ph}^{\prime \prime \prime \prime}\right), 7.18\left(1 \mathrm{H}\right.$, br s, $\left.H 4^{\prime \prime \prime}\right), 5.12\left(2 \mathrm{H}, \mathrm{s}, \mathrm{CH}_{2}\right), 2.42(6 \mathrm{H}$, $\left.\mathrm{s}, \mathrm{CH}_{3}\right) ;{ }^{13} \mathrm{C} \mathrm{NMR}\left(75 \mathrm{MHz}, \mathrm{CD}_{3} \mathrm{OD}\right): \delta 152.1,150.6,148.3$, $148.2,147.9,140.0,138.7,137.8,136.6,134.3,132.8,130.9$, 130.7, 130.1, 130.1, 130.1, 130.0, 129.7, 125.8, 60.5, 21.4. MS (ESI, $m / z)$ : 443 (97, $\left.\mathrm{M}^{+}-\mathrm{Br}\right), 364$ (100). HRMS (ESI-TOF, $\left.\mathrm{CH}_{3} \mathrm{OH}\right)$ calcd for $\mathrm{C}_{30} \mathrm{H}_{27} \mathrm{~N}_{4}$ : $[\mathrm{M}-\mathrm{Br}]^{+}$443.2236, found: 443.2228.

\subsubsection{1-\{N-Benzyl-N-[3-(3,5-dimethyl)phenyl-5-}

phenylpyrazin-2-yl]amino\}pyridinium bromide $(\mathbf{1 2 b})$

White solid (183 mg, 70\%, ethanol), mp $181-183{ }^{\circ} \mathrm{C}$; IR $(\mathrm{KBr}) \nu_{\max }\left(\mathrm{cm}^{-1}\right): 3000,1615,1529,1467,1399,1374$, 1226, 1184, 1158, 858, 766, 720, 692; ' ${ }^{\mathrm{H}} \mathrm{NMR}(300 \mathrm{MHz}$, $\left.\mathrm{CD}_{3} \mathrm{OD}\right): \delta 8.96\left(1 \mathrm{H}, \mathrm{s}, H 6^{\prime}\right), 8.92(2 \mathrm{H}, \mathrm{dd}, J=6.7$ and $1.3 \mathrm{~Hz}$, $H 2(6)), 8.54(1 \mathrm{H}, \mathrm{tt}, J=7.8$ and $1.3 \mathrm{~Hz}, H 4), 8.16(2 \mathrm{H}$, dd, $J=7.7$ and $\left.1.6 \mathrm{~Hz}, H 2^{\prime \prime \prime}\left(6^{\prime \prime \prime}\right)\right), 7.96(2 \mathrm{H}, \mathrm{dd}, J=7.8$ and $6.7 \mathrm{~Hz}$, $H 3(5)), 7.55\left(3 \mathrm{H}, \mathrm{m}, H 3^{\prime \prime \prime}\left(5^{\prime \prime \prime}\right)\right.$ and $\left.H 4^{\prime \prime \prime}\right), 7.37(2 \mathrm{H}$, br s, $H 2^{\prime \prime}\left(6^{\prime \prime}\right), 7.30\left(5 \mathrm{H}, \mathrm{m}, H 2^{\prime \prime \prime \prime}\left(6^{\prime \prime \prime \prime}\right), H 3^{\prime \prime \prime \prime}\left(5^{\prime \prime \prime \prime}\right)\right.$ and $\left.H 4^{\prime \prime \prime \prime}\right), 7.18$ $\left(1 \mathrm{H}\right.$, br s, $\left.\mathrm{H}^{\prime \prime \prime}\right), 5.13\left(2 \mathrm{H}, \mathrm{s}, \mathrm{CH}_{2}\right), 2.42\left(6 \mathrm{H}, \mathrm{s}, \mathrm{CH}_{3}\right) ;{ }^{13} \mathrm{C}$ NMR (75 MHz, $\left.\mathrm{CD}_{3} \mathrm{OD}\right): \delta 151.5,150.8,148.3,148.2,148.0$, 140.1, 138.4, 137.7, 136.7, 134.3, 132.3, 131.8, 131.2, 130.5, 130.2, 130.1, 129.7, 128.0, 127.4, 61.5, 21.3. MS (ESI, $\mathrm{m} / \mathrm{z}$ ): $443\left(20, \mathrm{M}^{+}-\mathrm{Br}\right), 364$ (100). HRMS (ESI-TOF, $\mathrm{CH}_{3} \mathrm{OH}$ ) calcd for $\mathrm{C}_{30} \mathrm{H}_{27} \mathrm{~N}_{4}$ : [M-Br] ${ }^{+}$443.2234, found: 443.2228 . 
5.6.3. $1-\{N-[5-(3,5-D i m e t h y l p h e n y l)-3-p h e n y l p y r a z i n-2-y l]-$

$\mathrm{N}$-[(3-methyl)benzyl]amino\} pyridinium bromide $(\mathbf{1 2 c})$

White solid (182 mg, 68\%, ethanol), mp $187-189^{\circ} \mathrm{C}$; IR (KBr) $\nu_{\max }\left(\mathrm{cm}^{-1}\right): 3104,3031,2917,1616,1471,1411$, $1370,1240,1160,1017,859,765,745,725,700,682,656$; ${ }^{1} \mathrm{H}$ NMR $\left(300 \mathrm{MHz}, \mathrm{CD}_{3} \mathrm{OD}\right): \delta 8.96\left(1 \mathrm{H}, \mathrm{s}, H 6^{\prime}\right), 8.92(2 \mathrm{H}$, $\mathrm{dd}, J=6.8$ and $1.3 \mathrm{~Hz}, H 2(6)), 8.51(1 \mathrm{H}, \mathrm{tt}, J=7.8$ and $1.3 \mathrm{~Hz}$, $H 4), 7.93(2 \mathrm{H}, \mathrm{dd}, J=7.8$ and $6.8 \mathrm{~Hz}, H 3(5)), 7.81(2 \mathrm{H}$, $\left.\mathrm{m}, H 2^{\prime \prime}\left(6^{\prime \prime}\right)\right), 7.78\left(2 \mathrm{H}\right.$, br s, $H 2^{\prime \prime \prime}\left(6^{\prime \prime \prime}\right), 7.55\left(3 \mathrm{H}, \mathrm{m}, H 3^{\prime \prime}\left(5^{\prime \prime}\right)\right.$ and $\left.H 4^{\prime \prime}\right), 7.16\left(3 \mathrm{H}, \mathrm{m}, H 4^{\prime \prime \prime \prime}, H 5^{\prime \prime \prime \prime}\right.$ and $\left.H 6^{\prime \prime \prime \prime}\right), 7.06(1 \mathrm{H}, \mathrm{br} \mathrm{s}$, $\left.H 4^{\prime \prime \prime}\right), 7.03\left(1 \mathrm{H}\right.$, br s, $\left.H 2^{\prime \prime \prime \prime}\right), 5.06\left(2 \mathrm{H}, \mathrm{s}, \mathrm{CH}_{2}\right), 2.42(6 \mathrm{H}, \mathrm{s}$, $\left.\mathrm{CH}_{3}\right), 2.27\left(3 \mathrm{H}, \mathrm{s}, \mathrm{CH}_{3}\right) ;{ }^{13} \mathrm{C}$ NMR $\left(75 \mathrm{MHz}, \mathrm{CD}_{3} \mathrm{OD}\right)$ : $\delta$ 152.3, 150.9, 148.6, 148.6, 148.2, 140.5, 140.2, 139.0, 138.1, 136.8, 134.4, 133.1, 131.6, 131.2, 131.1, 130.4, 130.3, 130.0, 130.0, 128.0, 126.1, 60.9, 21.7, 21.6. MS (ESI, $\mathrm{m} / \mathrm{z}): 457$ (100, $\mathrm{M}^{+}-\mathrm{Br}$ ), 378 (66). HRMS (ESI-TOF, $\mathrm{CH}_{3} \mathrm{OH}$ ) calcd for $\mathrm{C}_{31} \mathrm{H}_{29} \mathrm{~N}_{4}$ : [M-Br] ${ }^{+}$457.2392, found: 457.2384.

\subsection{4. $1-\{N-[5-(B e n z o[b]$ thiophen-3-yl)-3-phenylpyrazin-}

\section{2-yl]-N-benzylamino\} pyridinium bromide (12d)}

White solid (201 mg, 79\%, ethanol), mp $165-166^{\circ} \mathrm{C}$; IR $(\mathrm{KBr}) \nu_{\max }\left(\mathrm{cm}^{-1}\right): 3009,2931,1615,1512,1471,1456$, $1428,1408,1163,1058,760,732,702 ;{ }^{1} \mathrm{H}$ NMR $(500 \mathrm{MHz}$, $\left.\mathrm{CD}_{3} \mathrm{OD}\right): \delta 8.94(2 \mathrm{H}, J=6.9$ and $1.3 \mathrm{~Hz}, H 2(6)), 8.80(1 \mathrm{H}, \mathrm{s}$, $\left.H 6^{\prime}\right), 8.59\left(1 \mathrm{H}, \mathrm{dd}, J=6.1\right.$ and $\left.2.8 \mathrm{~Hz}, H 4^{\prime \prime \prime}\right), 8.43(1 \mathrm{H}, \mathrm{tt}, J=$ 7.8 and $1.3 \mathrm{~Hz}, \mathrm{H} 4), 8.30\left(1 \mathrm{H}, \mathrm{s}, H 2^{\prime \prime \prime}\right), 7.87(3 \mathrm{H}, \mathrm{m}, H 3(5)$ and $\left.7^{\prime \prime \prime}\right), 7.77\left(2 \mathrm{H}, \mathrm{dd}, J=8.0\right.$ and $\left.1.5 \mathrm{~Hz}, H 2^{\prime \prime}\left(6^{\prime \prime}\right)\right), 7.51(2 \mathrm{H}$, $\mathrm{tt}, J=7.3$ and $\left.1.5 \mathrm{~Hz}, \mathrm{H}^{\prime \prime}\left(5^{\prime \prime}\right)\right), 7.46(1 \mathrm{H}, \mathrm{tt}, J=7.3$ and $1.5 \mathrm{~Hz}$, $\left.\mathrm{H} 4^{\prime \prime}\right), 7.33\left(2 \mathrm{H}, \mathrm{m}, H 6^{\prime \prime \prime}\right.$ and $\left.7^{\prime \prime \prime}\right), 7.25\left(5 \mathrm{H}, \mathrm{m}, H 2^{\prime \prime \prime \prime}\left(6^{\prime \prime \prime \prime}\right)\right.$, $3^{\prime \prime \prime \prime}\left(5^{\prime \prime \prime \prime}\right)$ and $\left.4^{\prime \prime \prime \prime}\right), 4.99\left(2 \mathrm{H}, \mathrm{s}, \mathrm{CH}_{2}\right) ;{ }^{13} \mathrm{C}$ NMR $(75 \mathrm{MHz}$, $\left.\mathrm{CD}_{3} \mathrm{OD}\right): \delta$ 157.2, 149.6, 149.1, 148.1, 148.0, 147.1, 141.9, $140.1,140.1,138.0,137.5,134.0,132.7,130.9,130.6,130.2$, 130.0, 129.9, 129.6, 126.0, 125.9, 125.5, 123.7, 60.4. HRMS (ESI-TOF, $\mathrm{CH}_{3} \mathrm{OH}$ ) calcd for $\mathrm{C}_{30} \mathrm{H}_{23} \mathrm{~N}_{4} \mathrm{~S}: \quad[\mathrm{M}-\mathrm{Br}]^{+}$ 471.1643, found: 471.1634

\subsection{5. $1-\{N-[3-(B e n z o[b]$ thiophen-3-yl)-5-phenylpyrazin-}

2-yl]-N-[(2-bromo)benzyl]amino\}pyridinium bromide (12e)

Yellow solid (255 mg, $81 \%$, ethanol), $\mathrm{mp}>130{ }^{\circ} \mathrm{C}$ (dec); IR $(\mathrm{KBr}) \nu_{\max }\left(\mathrm{cm}^{-1}\right): 3053,1728,1612,1532,1471,1416,1332$, $1175,1027,766,739,694,508,472 ;{ }^{1} \mathrm{H}$ NMR $(300 \mathrm{MHz}$, $\left.\mathrm{CD}_{3} \mathrm{OD}\right): \delta 9.24\left(1 \mathrm{H}, \mathrm{s}, H 6^{\prime}\right), 8.38(2 \mathrm{H}, \mathrm{dd}, J=6.7$ and $1.5 \mathrm{~Hz}$, $H 2(6)), 8.23(1 \mathrm{H}, \mathrm{tt}, J=7.7$ and $1.5 \mathrm{~Hz}, H 4), 8.20(2 \mathrm{H}, \mathrm{dd}, J=$ 7.9 and $\left.1.8 \mathrm{~Hz}, H 2^{\prime \prime \prime}\left(6^{\prime \prime \prime}\right)\right), 7.94\left(1 \mathrm{H}, \mathrm{m}, H 4^{\prime \prime}\right), 7.82(1 \mathrm{H}, \mathrm{s}$, $\left.H 2^{\prime \prime}\right), 7.65(2 \mathrm{H}, \mathrm{dd}, J=7.7$ and $6.7 \mathrm{~Hz}, H 3(5)), 7.62(1 \mathrm{H}, \mathrm{m}$, $\left.H 7^{\prime \prime}\right), 7.55$ ( $4 \mathrm{H}, \mathrm{m}, H 3^{\prime \prime \prime}\left(5^{\prime \prime \prime}\right), H 4^{\prime \prime \prime}$ and $\left.H 3^{\prime \prime \prime \prime}\right), 7.44(1 \mathrm{H}$, ddd, $J=8.5,7.3$ and $1.5 \mathrm{~Hz}, H 5^{\prime \prime}$ or $\left.H 6^{\prime \prime}\right), 7.37(1 \mathrm{H}, \mathrm{ddd}, J=8.2$, 7.3 and $1.5 \mathrm{~Hz}, H 6^{\prime \prime}$ or $\left.H 5^{\prime \prime}\right), 7.28\left(3 \mathrm{H}, \mathrm{m}, H 4^{\prime \prime \prime \prime}, H 5^{\prime \prime \prime \prime}\right.$ and $\left.H 6^{\prime \prime \prime \prime}\right), 5.40\left(2 \mathrm{H}, \mathrm{s}, \mathrm{CH}_{2}\right) ;{ }^{13} \mathrm{C}$ NMR $\left(75 \mathrm{MHz}, \mathrm{CD}_{3} \mathrm{OD}\right)$ : $\delta 151.9,151.8,147.9,147.5,143.6,141.1,139.5,138.2,136.5$, 134.7, 134.0, 133.7, 132.7, 132.2, 131.4, 130.2, 129.8, 129.7, 129.4, 128.1, 126.6, 126.3, 126.0, 124.0, 123.7, 60.2. MS (ESI, $\mathrm{m} / \mathrm{z}$ ): 551/549 (100/86, $\left.\mathrm{M}^{+}-\mathrm{Br}\right), 472 / 470(51 / 46)$. HRMS (ESITOF, $\left.\mathrm{CH}_{3} \mathrm{OH}\right)$ calcd for $\mathrm{C}_{30} \mathrm{H}_{22} \mathrm{BrN}_{4} \mathrm{~S}$ : $[\mathrm{M}-\mathrm{Br}]^{+} 551.0728 /$ 549.0749, found: 551.0718/549.0734.
5.6.6. 1-\{N-[3-(Benzo[b]thiophen-3-yl)-5-(3,5dimethylphenyl)pyrazin-2-yl]-N-benzylamino\} pyridinium bromide (12f)

Yellow solid (226 mg, 78\%, ethanol), $\mathrm{mp}>129{ }^{\circ} \mathrm{C}$ (dec); IR $(\mathrm{KBr}) \nu_{\max }\left(\mathrm{cm}^{-1}\right): 3004,1613,1532,1471,1430,1371$, $1334,1237,1179,1042,854,763,732,700,669 ;{ }^{1} \mathrm{H}$ NMR $\left(300 \mathrm{MHz}, \mathrm{CD}_{3} \mathrm{OD}\right): \delta 9.10\left(1 \mathrm{H}, \mathrm{s}, H 6^{\prime}\right), 8.55(2 \mathrm{H}, \mathrm{dd}, J=$ 6.8 and $1.3 \mathrm{~Hz}, H 2(6)), 8.28(1 \mathrm{H}, \mathrm{tt}, J=7.7$ and $1.3 \mathrm{~Hz}, H 4)$, $8.00\left(1 \mathrm{H}, \mathrm{m}, H 4^{\prime \prime}\right), 7.98\left(1 \mathrm{H}, \mathrm{s}, H 2^{\prime \prime}\right), 7.79(2 \mathrm{H}, \mathrm{br} \mathrm{s}$, $\left.H 2^{\prime \prime \prime}\left(6^{\prime \prime \prime}\right)\right), 7.73(2 \mathrm{H}$, dd, $J=7.7$ and $6.8 \mathrm{~Hz}, H 3(5)), 7.69$ $\left(1 \mathrm{H}, \mathrm{m}, H 7^{\prime \prime}\right), 7.47\left(2 \mathrm{H}, \mathrm{m}, H 5^{\prime \prime}\right.$ and $\left.H 6^{\prime \prime}\right), 7.23(6 \mathrm{H}, \mathrm{m}$, $H 2^{\prime \prime \prime \prime}\left(6^{\prime \prime \prime \prime}\right), H 3^{\prime \prime \prime \prime}\left(5^{\prime \prime \prime \prime}\right), H 4^{\prime \prime \prime \prime}$ and $\left.H 4^{\prime \prime \prime}\right), 5.20\left(2 \mathrm{H}, \mathrm{s}, \mathrm{CH} \mathrm{H}_{2}\right)$, $2.42\left(6 \mathrm{H}, \mathrm{s}, \mathrm{CH}_{3}\right) ;{ }^{13} \mathrm{C}$ NMR $\left(75 \mathrm{MHz}, \mathrm{CD}_{3} \mathrm{OD}\right): \delta 152.0$, 151.6, 147.8, 147.6, 143.2, 141.3, 140.0, 139.3, 139.2, 138.2, $136.5,134.3,133.0,132.8,130.9,130.2,130.1,129.6,126.6$, 126.0, 125.8, 124.1, 123.9, 60.5, 21.4. MS (ESI, $m / z)$ : 499 (37, $\left.\mathrm{M}^{+}-\mathrm{Br}\right), 420$ (100).

\subsection{Preparation of unsymmetrical benzyl(3,5-diaryl- pyrazin-2-yl)amines (13)}

General method. Zinc dust (500 mg) was added to a solution of the corresponding pyridinium salt $\mathbf{1 2}(100 \mathrm{mg})$ in glacial acetic acid $(25 \mathrm{~mL})$ and the mixture was stirred at room temperature for $48 \mathrm{~h}$. During this period a slight change in the colour of the solution was observed. Once the reaction had finished, the remaining solid was removed by filtration and the solvent evaporated. The residue was dissolved in a mixture of ethyl acetate $(10 \mathrm{~mL})$ and an aqueous solution of sodium hydroxide $(20 \%)(5 \mathrm{~mL})$. The organic layer was separated and the aqueous phase was re-extracted with ethyl acetate $(2 \times 5 \mathrm{~mL})$. The combined organic layers were extracted with hydrochloric acid $(20 \%)(2 \times 5 \mathrm{~mL})$ and the aqueous phase, containing the aminoazine hydrochloride, was then basified to $\mathrm{pH}=10$ using solid sodium hydroxide. The free amine was extracted using ethyl acetate $(3 \times 10 \mathrm{~mL})$. The combined organic phases were washed with brine, dried over $\mathrm{MgSO}_{4}$, filtered and evaporated to dryness. The residue was purified by column chromatography on silica gel, using dichloromethane as eluent, and the corresponding benzyl(3,5-diarylpyrazin2-yl)amine 13 was crystallized from a suitable solvent.

\subsubsection{N-Benzyl-N-[5-(3,5-dimethyl)phenyl-3-phenyl- pyrazin-2-yllamine (13a)}

White solid (43 mg, 62\%, ethyl acetate/hexane) $\mathrm{mp} 102-$ $104{ }^{\circ} \mathrm{C}$, IR $(\mathrm{NaCl}) \nu_{\max }\left(\mathrm{cm}^{-1}\right): 3435,3028,2917,1668$, $1604,1580,1506,1359,1218,1163,1027,850,742$, 699; ${ }^{1} \mathrm{H}$ NMR $\left(300 \mathrm{MHz}, \mathrm{CDCl}_{3}\right): \delta 8.45\left(1 \mathrm{H}, \mathrm{s}, H 6^{\prime}\right), 7.74(2 \mathrm{H}$, dd, $J=8.2$ and $\left.1.8 \mathrm{~Hz}, H 2^{\prime \prime}\left(6^{\prime \prime}\right)\right), 7.53\left(2 \mathrm{H}\right.$, br s, $\left.H 2^{\prime \prime \prime}\left(6^{\prime \prime \prime}\right)\right)$, $7.46\left(3 \mathrm{H}, \mathrm{m}, H 3^{\prime \prime}\left(5^{\prime \prime}\right)\right.$ and $\left.H 4^{\prime \prime}\right), 7.28\left(5 \mathrm{H}, \mathrm{m}, H-\mathrm{Ph}^{\prime \prime \prime \prime}\right), 6.97$ $\left(1 \mathrm{H}\right.$, br s, $\left.H 4^{\prime \prime \prime}\right), 5.28(1 \mathrm{H}, \mathrm{t}, J=5.9 \mathrm{~Hz}, \mathrm{NH}), 4.67(2 \mathrm{H}$, d, $\left.J=5.9 \mathrm{~Hz}, \mathrm{CH}_{2}\right), 2.36\left(6 \mathrm{H}, \mathrm{s}, \mathrm{CH}_{3}\right) ;{ }^{13} \mathrm{C}$ NMR $(75 \mathrm{MHz}$, $\left.\mathrm{CDCl}_{3}\right): \delta 150.6\left(C 2^{\prime}\right), 141.3\left(C 5^{\prime}\right), 140.3\left(C 3^{\prime}\right), 139.1\left(C 1^{\prime \prime \prime \prime}\right)$, $138.2\left(C 6^{\prime}\right), 137.7\left(C 3^{\prime \prime \prime}\left(5^{\prime \prime \prime}\right)\right), 137.3\left(C 1^{\prime \prime \prime}\right.$ or $\left.C 1^{\prime \prime}\right), 137.2\left(C 1^{\prime \prime}\right.$ or $\left.C 1^{\prime \prime \prime}\right), 129.5\left(C 4^{\prime \prime \prime}\right), 129.2\left(C 3^{\prime \prime}\left(5^{\prime \prime}\right)\right), 129.0\left(C 4^{\prime \prime}\right), 128.6$ $\left(C 3^{\prime \prime \prime \prime}\left(5^{\prime \prime \prime \prime}\right)\right), \quad 128.5 \quad\left(C 2^{\prime \prime}\left(6^{\prime \prime}\right)\right), \quad 127.5 \quad\left(C 2^{\prime \prime \prime \prime}\left(6^{\prime \prime \prime \prime}\right)\right), \quad 127.3$ $\left(C 4^{\prime \prime \prime \prime}\right), 123.5\left(C 2^{\prime \prime \prime}\left(6^{\prime \prime \prime}\right)\right), 45.4\left(C_{2}\right), 21.4\left(C_{3}\right)$. HRMS 
(ESI-TOF, $\mathrm{CH}_{3} \mathrm{OH}$ ) calcd for $\mathrm{C}_{25} \mathrm{H}_{24} \mathrm{~N}_{3}$ : $[\mathrm{M}+\mathrm{H}]^{+}$366.1970, found: 366.1992 .

\subsubsection{N-Benzyl-N-[3-(3,5-dimethyl)phenyl-5-}

phenylpyrazin-2-yl]amine (13b)

White solid (39 mg, 57\%, ethyl acetate/hexane) mp 91$93{ }^{\circ} \mathrm{C}, \mathrm{IR}(\mathrm{NaCl}) \nu_{\max }\left(\mathrm{cm}^{-1}\right): 3432,3030,2920,1602,1513$, $1488,1195,1163,1028,856,764,695 ;{ }^{1} \mathrm{H}$ NMR $(300 \mathrm{MHz}$, $\left.\mathrm{CDCl}_{3}\right): \delta 8.45\left(1 \mathrm{H}, \mathrm{s}, H 6^{\prime}\right), 7.93(2 \mathrm{H}, \mathrm{dd}, J=7.8$ and $1.5 \mathrm{~Hz}$, $\left.H 2^{\prime \prime \prime}\left(6^{\prime \prime \prime}\right)\right), 7.41\left(3 \mathrm{H}, \mathrm{m}, H 3^{\prime \prime \prime}\left(5^{\prime \prime \prime}\right)\right.$ and $\left.H 4^{\prime \prime \prime}\right), 7.32(7 \mathrm{H}, \mathrm{m}$, $H 2^{\prime \prime}\left(6^{\prime \prime}\right)$ and $\left.H-\mathrm{Ph}^{\prime \prime \prime \prime}\right), 7.05\left(1 \mathrm{H}\right.$, br s, $\left.H 4^{\prime \prime}\right), 5.33(1 \mathrm{H}, \mathrm{t}, J=$ $5.5 \mathrm{~Hz}, \mathrm{NH}), 4.67\left(2 \mathrm{H}, \mathrm{t}, J=5.5 \mathrm{~Hz}, \mathrm{CH}_{2}\right), 1.23\left(6 \mathrm{H}, \mathrm{s}, \mathrm{CH}_{3}\right)$; ${ }^{13} \mathrm{C}$ NMR $\left(75 \mathrm{MHz}, \mathrm{CDCl}_{3}\right): \delta 150.8\left(C 2^{\prime}\right), 140.9\left(C 5^{\prime}\right.$ or $\left.C 1^{\prime \prime \prime \prime}\right), 140.8\left(C 1^{\prime \prime \prime \prime \prime}\right.$ or $\left.C 5^{\prime}\right), 139.2\left(C 3^{\prime}\right), 138.8\left(C 6^{\prime}\right), 137.4$ $\left(C 3^{\prime \prime}\left(5^{\prime \prime}\right)\right.$ and $C 1^{\prime \prime \prime}$ or $\left.C 1^{\prime \prime}\right), 137.0\left(C 1^{\prime \prime}\right.$ or $\left.C 1^{\prime \prime \prime}\right), 130.7\left(C 4^{\prime \prime}\right)$, $128.7\left(C 3^{\prime \prime \prime}\left(5^{\prime \prime \prime}\right)\right.$ or $\left.C 3^{\prime \prime \prime \prime}\left(5^{\prime \prime \prime \prime}\right)\right), 128.6\left(C 3^{\prime \prime \prime \prime}\left(5^{\prime \prime \prime \prime}\right)\right.$ or $\left.C 3^{\prime \prime \prime}\left(5^{\prime \prime \prime}\right)\right), 127.7\left(C 4^{\prime \prime \prime}\right), 127.4\left(C 2^{\prime \prime \prime}\left(6^{\prime \prime \prime}\right)\right), 127.2\left(C 4^{\prime \prime \prime \prime}\right), 126.1$ $\left(C 2^{\prime \prime \prime}\left(6^{\prime \prime \prime}\right)\right.$ or $\left.C 2^{\prime \prime \prime \prime \prime}\left(6^{\prime \prime \prime \prime}\right)\right), 125.6\left(C 2^{\prime \prime \prime \prime}\left(6^{\prime \prime \prime \prime}\right)\right.$ or $\left.C 2^{\prime \prime \prime}\left(6^{\prime \prime \prime}\right)\right), 45.4$ $\left(\mathrm{CH}_{2}\right), 21.4\left(\mathrm{CH}_{3}\right)$. HRMS (ESI-TOF, $\mathrm{CH}_{3} \mathrm{OH}$ ) calcd for $\mathrm{C}_{25} \mathrm{H}_{24} \mathrm{~N}_{3}:[\mathrm{M}+\mathrm{H}]^{+}$366.1970, found: 366.1987 .

\subsubsection{N-[5-(Benzo[b]thiophen-3-yl)-5-phenylpyrazin-2-yl]- $\mathrm{N}$-benzylamine $(\mathbf{1 3 c})$}

Yellow oil (45 mg, 63\%); IR ( $\mathrm{NaCl}): \nu_{\max }\left(\mathrm{cm}^{-1}\right): 3434$, $3059,2921,1577,1524,1494,1452,1425,1349,1218$, 1161, 1027, 759, 734, 700; ${ }^{1} \mathrm{H}$ NMR $\left(500 \mathrm{MHz}, \mathrm{CDCl}_{3}\right)$ : $\delta 8.45\left(1 \mathrm{H}, \mathrm{s}, H 6^{\prime}\right), 8.42\left(2 \mathrm{H}, \mathrm{m}, H 4^{\prime \prime \prime}\right), 7.88\left(1 \mathrm{H}, \mathrm{m}, H 7^{\prime \prime \prime}\right)$, $7.78\left(2 \mathrm{H}, \mathrm{m}, H 2^{\prime \prime}\left(6^{\prime \prime}\right)\right), 7.51\left(2 \mathrm{H}, \mathrm{m}, H 3^{\prime \prime}\left(5^{\prime \prime}\right)\right), 7.44(1 \mathrm{H}, \mathrm{m}$, $\left.\mathrm{H} 4^{\prime \prime}\right), 7.38\left(2 \mathrm{H}, \mathrm{m}, H 5^{\prime \prime \prime}\right.$ and $\left.H 6^{\prime \prime \prime}\right), 7.35\left(4 \mathrm{H}, \mathrm{m}, H 2^{\prime \prime \prime \prime}\left(6^{\prime \prime \prime \prime}\right)\right.$ and $\left.H 3^{\prime \prime \prime \prime}\left(5^{\prime \prime \prime \prime}\right)\right), 7.27\left(1 \mathrm{H}, \mathrm{m}, H 4^{\prime \prime \prime \prime}\right), 5.36(1 \mathrm{H}, \mathrm{t}, J=5.6 \mathrm{~Hz}$, $\mathrm{NH}), 4.70\left(2 \mathrm{H}, \mathrm{d}, J=5.6 \mathrm{~Hz}, \mathrm{CH}_{2}\right) ;{ }^{13} \mathrm{C}$ NMR $(75 \mathrm{MHz}$, $\left.\mathrm{CDCl}_{3}\right): \delta 150.5\left(C 2^{\prime}\right), 140.8\left(C 7 \mathrm{a}^{\prime \prime \prime}\right), 140.2\left(C 3^{\prime}\right), 139.3$ $\left(C 6^{\prime}\right), 139.0\left(C 3^{\prime \prime \prime}\right), 138.7\left(C 3 \mathrm{a}^{\prime \prime \prime}\right), 137.4\left(C 1^{\prime \prime}\right), 137.0\left(C 1^{\prime \prime \prime \prime}\right)$, $134.0\left(C 5^{\prime}\right), 129.2\left(C 2^{\prime \prime}\left(6^{\prime \prime}\right)\right.$ or $\left.3^{\prime \prime}\left(5^{\prime \prime}\right)\right), 129.1\left(C 4^{\prime \prime}\right), 128.6$ $\left(C 3^{\prime \prime}\left(5^{\prime \prime}\right)\right.$ or $\left.C 2^{\prime \prime}\left(6^{\prime \prime}\right)\right), 128.3\left(C 2^{\prime \prime \prime \prime}\left(6^{\prime \prime \prime \prime}\right)\right.$ or $\left.C 3^{\prime \prime \prime \prime}\left(5^{\prime \prime \prime \prime \prime}\right)\right), 127.5$ $\left(C 3^{\prime \prime \prime \prime}\left(5^{\prime \prime \prime \prime}\right)\right.$ or $\left.C 2^{\prime \prime \prime \prime}\left(6^{\prime \prime \prime \prime}\right)\right)$, $127.3\left(C 4^{\prime \prime \prime \prime}\right), 124.4\left(C 4^{\prime \prime \prime}\right.$ or $\left.C 6^{\prime \prime \prime}\right)$, $124.4\left(C 6^{\prime \prime \prime}\right.$ or $\left.C 4^{\prime \prime \prime}\right), 124.0\left(C 2^{\prime \prime \prime}\right.$ or $\left.C 5^{\prime \prime \prime}\right), 123.8,\left(C 5^{\prime \prime \prime}\right.$ or $\left.C 2^{\prime \prime \prime}\right), 122.7\left(C 7^{\prime \prime \prime}\right), 45.4\left(\mathrm{CH}_{2}\right)$. HRMS (APCI-TOF, $\left.\mathrm{CH}_{3} \mathrm{OH}\right)$ calcd for $\mathrm{C}_{25} \mathrm{H}_{20} \mathrm{~N}_{3} \mathrm{~S}$ : $[\mathrm{M}+\mathrm{H}]^{+} 394.1378$, found: 394.1388.

\section{Acknowledgements}

The authors wish to thank the Comisión Interministerial de Ciencia y Tecnología (CICYT-BQU2001-1508 and CTQ200508902) and the Universidad de Alcalá (UAH GC2005/006) for financial support and the Ministerio de Educación y Ciencia (MEC) for two studentships (M.J.R. and R.C.). We also thank Professor Mijail Galajov for his assistance in the NMR study.

\section{References and notes}

1. Pozharskii, A. F.; Soldatenkov, A. T.; Katrizky, A. R. Heterocycles in Life and Society; Wiley: New York, NY, 1997.

2. (a) Collins, I. J. Chem. Soc., Perkin Trans. 1 2000, 2845-2861; (b) Li, J. J.; Gribble, G. W. Palladium in Heterocyclic Chemistry; Pergamon: Amsterdam, 2000; (c) Agrofoglio, L. A.; Gillaizeau, I.; Saito, Y. Chem
Rev. 2003, 103, 1875-1916; (d) de Meijere, A.; Diederich, F.; MetalCatalysed Cross-Coupling Reactions, 2nd ed.; Wiley-VCH: Weinheim, 2004; Vols. 1 and 2; (e) Kirch, G.; Hesse, S.; Comel, A. Curr. Org. Synth. 2004, 1, 47-63; (f) Corbet, J.-P.; Mignani, G. Chem. Rev. 2006, 106, 2651-2710.

3. (a) Collins, I. J. Chem. Soc., Perkin Trans. 1 2002, 1921-1940; (b) Schröter, S.; Christoph, S.; Bach, T. Tetrahedron 2005, 61, 2245-2267; (c) Large, J. M.; Clarke, M.; Williamson, D. M.; McDonald, E.; Collins, I. Synlett 2006, 861-864.

4. (a) Miyaura, N.; Suzuki, A. Chem.Rev. 1995, 95, 2457-2495; (b) Suzuki, A. J. Organomet. Chem. 1999, 576, 147-168; (c) Kotha, S.; Lahiri, L.; Kashinath, D. Tetrahedron 2002, 58, 9633-9695.

5. (a) Nájera, C.; Gil-Moltó, J.; Karlström, S. Adv. Synth. Catal. 2004, 346, 1798-1811; (b) Lu, G.; Fanzén, R.; Zahng, Q.; Xu, Y. Tetrahedron Lett. 2005, 46, 4255-4259; (c) Leadbeater, N. E. Chem. Commun. 2005, 2881-2902; (d) Li, J.-H.; Zhu, Q.-M.; Xie, Y.-X. Tetrahedron 2006, 62, 10888-10895; (e) Kudo, N.; Perseghini, M.; Fu, G. C. Angew. Chem., Int. Ed. 2006, 45, 1282-1284; (f) Billingsley, K. L.; Anderson, K. W.; Buchwald, S. L. Angew. Chem., Int. Ed. 2006, 45, 34843488 .

6. Vaquero, J. J.; Alvarez-Builla, J. Advances in Nitrogen Heterocycles; JAI: Stamford, 2000; Vol. 4, pp 159-250.

7. Ollis, D. W.; Stanforth, S. P. Tetrahedron 1985, 41, 2239-2329.

8. (a) Carceller, R.; García-Navío, J. L.; Izquierdo, M. L.; Alvarez-Builla, J. Tetrahedron Lett. 1993, 34, 2019-2020; (b) Carceller, R.; García-Navío, J. L.; Izquierdo, M. L.; Alvarez-Builla, J.; Fajardo, M.; Gómez-Sal, P.; Gago, F. Tetrahedron 1994, 50, 4995-5012; (c) Burgos, C.; Delgado, F.; García-Navío, J. L.; Izquierdo, M. L.; Alvarez-Builla, J. Tetrahedron 1995, 51, 8649-8654; (d) García de Viedma, A.; Martinez-Barrasa, V.; Burgos, C.; Izquierdo, M. L.; Alvarez-Builla, J. J. Org. Chem. 1999, 64, 1007-1010; (e) Martínez-Barrasa, V.; Delgado, F.; Burgos, C.; GarcíaNavío, J. L.; Izquierdo, M. L.; Alvarez-Builla, J. Tetrahedron 2000, 56, 2481-2490; (f) De la Rosa, R.; Martinez-Barrasa, V.; Burgos, C.; Alvarez-Builla, J. Tetrahedron Lett. 2000, 41, 5837-5840; (g) Reyes, M. J.; Delgado, F.; Izquierdo, M. L.; Alvarez-Builla, J. Tetrahedron 2002, 58, 8573-8579; (h) Reyes, M. J.; Burgos, C.; Izquierdo, M. L.; Alvarez-Builla, J. Tetrahedron 2004, 60, 1093-1097; (i) Castillo, R.; Izquierdo, M. L.; Alvarez-Builla, J. Tetrahedron Lett. 2007, 48, 58995903.

9. (a) Matínez-Barrasa, V.; García de Viedma, A.; Burgos, C.; AlvarezBuilla, J. Org. Lett. 2000, 2, 3933-3935; (b) Nuñez, A.; García de Viedma, A.; Martínez-Barrasa, V.; Burgos, C.; Alvarez-Builla, J. Synlett 2002, 1093-1096; (c) Nuñez, A.; Sánchez, A.; Burgos, C.; Alvarez-Builla, J. Tetrahedron 2004, 60, 6217-6224; (d) Sánchez, A.; Nuñez, A.; Alvarez-Builla, J.; Burgos, C. Tetrahedron 2004, 60, 11843-11850; (e) Sánchez, A.; Nuñez, A.; Burgos, C.; AlvarezBuilla, J. Tetrahedron Lett. 2006, 47, 8343-8346; (f) Nuñez, A.; Sánchez, A.; Burgos, C.; Alvarez-Builla, J. Tetrahedron 2007, 63, 6774-6783.

10. (a) Reyes, M. J.; Izquierdo, M. L.; Alvarez-Builla, J. Tetrahedron Lett. 2004, 45, 8713-8715; (b) Reyes, M. J.; Castillo, R.; Izquierdo, M. L.; Alvarez-Builla, J. Tetrahedron Lett. 2006, 47, 6457-6460.

11. (a) Sadighi, J. P.; Singer, R. A.; Buchwald, S. L. J. Am. Chem. Soc. 1998 , 120, 4960-4976; (b) Garnier, E.; Audoux, J.; Pasquinet, E.; Suzenet, F.; Poullain, D.; Lebret, B.; Guillaumet, G. J. Org. Chem. 2004, 69, 78097815 .

12. (a) Nakamura, H.; Aizawa, M.; Takeuchi, D.; Murai, A.; Shimoura, O. Tetrahedron Lett. 2000, 41, 2185-2188; (b) Wu, C.; Nakamura, H.; Murai, A.; Shimoura, O. Tetrahedron Lett. 2001, 42, 2997-3000; (c) Jiang, B.; Yang, C.-G.; Xiong, W.-N.; Wang, J. Bioorg. Med. Chem. 2001, 9, 1149-1154; (d) Adamczyk, M.; Akireddy, S. R.; Johnson, D. D.; Mattingly, P. G.; Pan, Y.; Reddy, R. E. Tetrahedron 2003, 59, 8129-8142; (e) Saito, R.; Inoue, C.; Katoh, A. Heterocycles 2004, 63, 759-764; (f) Itoh, T.; Kato, S.; Nonoyama, N.; Wada, T.; Maeda, K.; Mase, T.; Zhao, M. M.; Song, J. Z.; Tschaen, D. M.; McNamara, J. M. Org. Process Res. Dev. 2006, 10, 822-828.

13. (a) Dubuisson, M. L. N.; Rees, J. F.; Marchand-Brynaert, J. Mini-Rev. Med. Chem. 2004, 4, 421-435; (b) Teranishi, K. Bioorg. Chem. 2007, 
35, 82-111; (c) Yang, C.-G.; Liu, G.; Jiang, B. J. Org. Chem. 2002, 67, 9392-9396; (d) Cavalier, J.-F.; Burton, M.; De Tollenaere, C.; Dussart, F.; Marchand, C.; Rees, J.-F.; Marchand-Brynaert, J. Synthesis 2001, 768772; (e) Jeanjot, P.; Bruyneel, F.; Arrault, A.; Gharbi, S.; Cavalier, J.-F.;
Abels, A.; Marchand, C.; Touillaux, R.; Rees, J.-F.; Marchand-Brynaert, J. Synthesis 2003, 513-522.

14. Birault, V.; Harris, J. C. WO 2005/003101, January 13, 2005.

15. Cai, D.; Larsen, R. D.; Reider, P. J. Tetrahedron Lett. 2002, 43, 4285-4287. 